NBSIR 82-2607

Center for Materials Science

\title{
Polymer
}

Science and

Standards

Division

\section{Annual Report 1982}

U.S. DEPARTMENT

OF COMMERCE

National Bureau

of Standards 



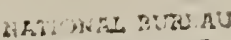

c) ctritsture 


\section{Polymer Science and Standards Division}

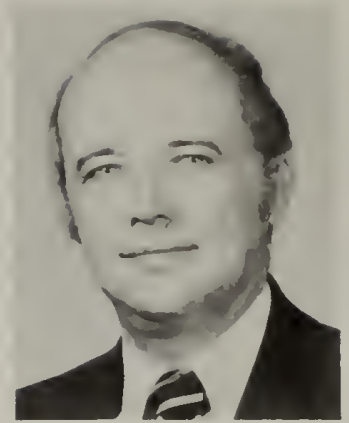

RONALD K. EBY, Chief. Dr. Eby was born in Reading, Pennsylvania. He earned the Sc.B. in physics at Lafayette College and the Sc.M. ad Ph.D. at Brown University. Before joining NBS in 1963, he conducted polymers research in the Plastics Department of E.I. du Pont de Nemours \& Co., Inc.

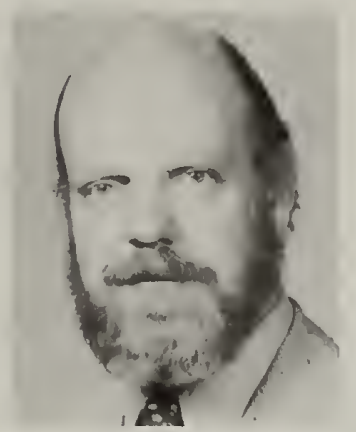

MARTIN G. BROADHURST, Acting Deputy Chief. Dr. Broadhurst was born in Washington, D.C., and earned degrees in physics at Western Maryland College and Pennsylvania State University. After teaching at Pennsylvania State, he joined NBS in 1960.

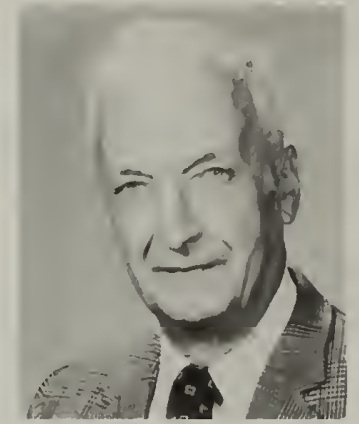

ANTON PETERLIN, Assistant Chief. Dr. Peterlin was born in Yugoslavia. He earned the M.S. in mathematics at the University of Ljubljana and Ph.D. in physics at the University of Berlin. From 1939 to 1960 he was professor of physics in Ljubljana and from 1949 to 1959 was head of the Josef Stefan Institute. Before joining NBS in 1973, he was director of the Camille Dreyfus Laboratory.

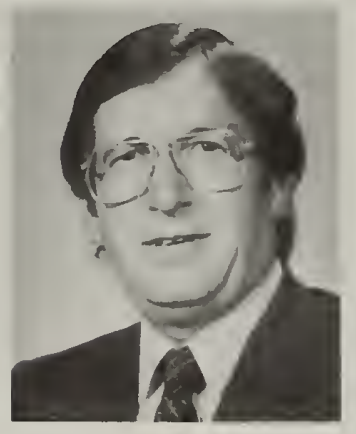

G. THOMAS DAVIS, Acting Group Leader, Electrical Properties. Dr. Davis was born in Montour Falls, New York. He earned degrees in chemical engineering at Cornell University and physical chemistry at Princeton University. Prior to joining NBS in 1964, he worked for Esso Research and Engineering Co. and taught at the University of Virginia.

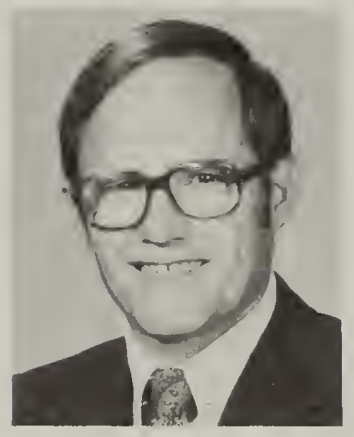

BRUNO M. FANCONI, Group Leader, Structure and Properties. Dr. Fanconi was born in Merced, California. He earned degrees in physical chemistry from the University of California at Los Angeles and the University of Washington. He joined the staff of NBS in 1971.

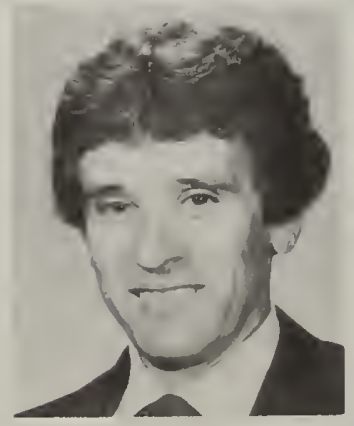

ISAAC C. SANCHEZ, Group Leader, Characterization and Standards. Dr. Sanchez was born in San Antonio, Texas. He earned degrees in physical chemistry at St. Marys University (Texas) and at the University of Delaware. Prior to joining the NBS staff in 1977, he worked at the Xerox Research Laboratories and was on the Polymer Science and Engineering faculty at the University of Massachusetts.

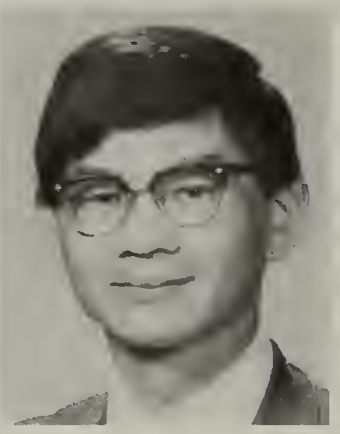

FRANCIS W. WANG, Acting Group Leader, Chemical Durability. Dr. Wang was born in Peikang, Taiwan. He earned the B.S. and M.S. in chemical engineering at California Institute of Technology and the Ph.D. in physical chemistry at the University of California, in San Diego. He joined the staff of NBS in 1972 after a postdoctoral period at the Polytechnic Institute of New York.

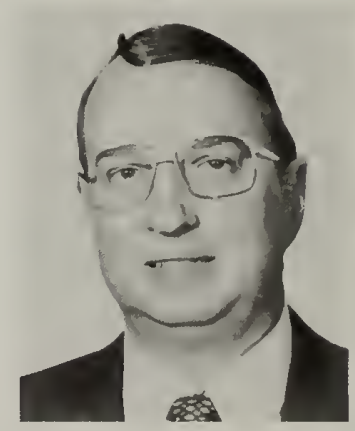

JOHN A. TESK, Acting Group Leader, Dental and Medical Materials. Dr. Tesk was born in Chicago, Illinois. He holds the degrees of B.S., M.S. in Metallurgy and Ph.D. in Materials Science from Northwestern University. Prior to joining NBS in 1978, he was at the University of Illinois, Chicago, on the staff at Argonne National Laboratory, Director of R\&D with the Dental Division of Howmedica, Inc. and Director of Education Services at IGT. 
BhW WR- 2:RU.AY

OP OTARPNALE

infmis?

MAY

21983

POLYMER SCIENCE AND STANDARDS DIVISION

Annual Report 1982

U.S. Department of Commerce National Bureau of Standards Polymers B1dg. (224), A305

Washington, DC 20234

(301) 921-3734

March 1983 



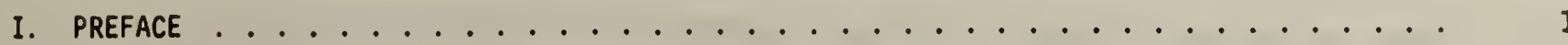

II. POLYMERS STANDARDS FOR PROCESS CONTROL AND EQUITY . . . . . . . . . . . . . 6

I. C. SANCHEZ - TASK LEADER

- Self-Calibrating Gel Permeation Chromatography; P. H. Verdier . . . . . . . . 6

- Liquid Size Exclusion Chromatography; H. L. Wagner and J. D. Barnes . . . . . . . 6

- Limiting Viscosity Number of Ultrahigh Molecular Weight Polyethylene; H. L. Wagner and J. G. Dillon ...........................

- Fractionation of Ultrahigh Molecular Weight Polyethylene; H. L. Wagner . . . .

- Analysis of Ultracentrifugation Interference Patterns with Image Digitizer Application to Molecular Weight Determination of SRM 1478 Polystyrene; F. W. Wang and F. L. McCrackin.

- Standards for Gas Transmission Measurements; J. D. Barnes ..............

- Diffusion of Polymers in Semidilute Solutions by Dynamic Light Scattering; E. J. Amis, C. C. Han, and Y. Matsuchita ........................ 1

- Forced Rayleigh Scattering; E. J. Amis and C. C. Han . . . . . . . . . . . . . .

- Concentration and Polydispersity Dependence of the Static Structure Factor $S(q, c)$ and Dynamic Characteristic Frequency, $\Omega(q, c)$ in Dilute Polymer Solutions; $A$. $Z$.

Akcasu, B. Hammouda, T. P. Lodge, and C. C. Han ....................

- Single Chain Conformation in Phase Separated Diblock Copolymer Systems; H. Hasegawa, E. J. Amis, T. P. Lodge, and C. C. Han ......................

- Small Angle Neutron Scattering From Block Copolymers of h-styrene and d-styrene; T. P. Lodge, Y. Matsuchita, E. J. Amis, and C. C. Han . . . . . . . . . . . . . . 12

- Static Light Scattering; C. C. Han, H. L. Wagner, and D. B. Minor . . . . . . . . 12

- Dynamics of Polymer Chains; P. H. Verdier and D. E. Kranbuehl . . . . . . . . . . 12

- Monte Carlo Calculations of the Hydrodynamic Radii of Polymers; F. L. McCrackin . . 13

- Span of an Adsorbed Random Flight Chain Calculated on the Basis of the deGennes Mode l; R. J. Rubin .............................

- Validation of the "Weak Adsorption" Diffusion Equation Plus Boundary Condition Model of deGennes; R. J. Rubin ...........................

- A New Equation of State Model for Polymers; I. C. Sanchez . . . . . . . . . . . . 14

- ASTM Activities; J. D. Barnes . . . . . . . . . . . . . . . . . . . . . 14

- Rubber and Rubber Compounding Standard Reference Materials; G. B. McKenna and G. W. Bullman ................................

\section{B. M. FANCONI - TASK LEADER}

- Molecular Processes Associated with Mechanical Deformation and Failure; B. M. Fanconi, D. L. VanderHart, S. J. Kryder, E. E. Toth, K. L. DeVries, G. A. Bohme, and V. D. Mochel .........................

- The Distribution of Stresses in Crazes at Crack Tips; E. Passaglia 
- A New Test Method for Environmental Stress-Crack Resistarice; J. M. Crissman and

L. J. Zapas . . . . . . . . . . . . . . . . . . . . . . . . . . . .

- Creep and Recovery of UHMWPE at Very Small Deformations: A New One-Dimensional Constitutive Equation; L. J. Zapas and J. M. Crissman ...............

- A Study of the Morphology and Mechanical Properties, and The $1 r$ Dependence on Thermal History and Processing of Ultra High Molecular Weight Polyethylene; J. M. Crissman and F. Khoury .................

- The Effects of Mechanical Deformation on Glassy Polymers; G. B. McKenna and

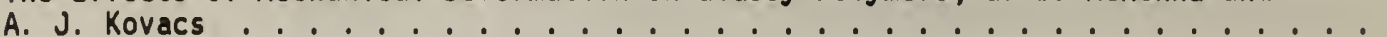

- Mechanical Relaxation of Liner Materials in Acetic Acid; J. C. Phillips . . . . .

- Crystal Growth Kinetics and the Lateral Growth Habits of Polyethylene Crystals; E. Passaglia and F. Khoury ........................

- The Morphology of Polyethylene Crystals Grown From Solution; F. A. Khoury and L. H. Bolz . . . . . . . . . . . . . . . . . . .

- The Energies of (110) and (200) Folds in Polyethylene Crystals; F. A. Khoury,

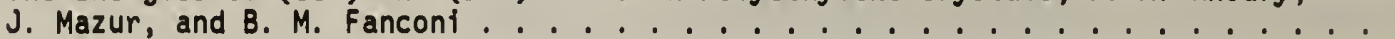

- Durability of Elastomers Used in Geothermal Energy Production; R. W. Penn . . . . .

IV. PROCESSING AND RELIABILITY OF POLYMER COMPOSITES . . . . . . . . . . . . . . . . . . . 31

B. M. FANCONI - TASK LEADER

- Failure Behavior of Particle Reinforced Polymer Composites; D. L. Hunston, A. J. Kinloch, and G. W. Bullman . . . . . . . . . . . . . . . . . . . .

- Interlaminar Fracture of Composites; D. L. Hunston, G. W. Bullman, and J. A. Kousky . . . . . . . . . . . . . . . . . . . . . . . . . . .

- A Non-Destructive Technique to Monitor Cure of Adhesive and Composite Resins;

D. L. Hunston and J. A. Koutsky . . . . . . . . . . . . . . . . . . 32

- Measurement of Microvoid and Microcracks in Composite Materials Using Small Angle X-ray Scattering Techniques; W. L. Wu . . . . . . . . . . . . . . . . . .

- Industrial Research Associate Program on Thermal and Spectroscopic Properties of Polymer-Based Composite Materials; B. N. Ranganathan, J. H. Flynn, and B. M.

Fancont . . . . . . . . . . . . . . . . . . . . . . . . . . . .

- Analysis of Rapid Thermoset Resin Curing; G. A. Senich . . . . . . . . . . . . 35

- Degree of Polymerization of Thermoset Resins Used in Composites; W. L. Wu and

B. M. Fanconi.... . . . . . . . . . . . . . . . . . . . . . . . .

V. ADDITIVE MIGRATION AND CHEMICAL DURABILITY OF POLYMERS .

F. W. WANG - TASK LEADER

- Hydrolytic Degradation of Polyester Polyurethane Elastomers; D. W. Brown, R. E. Lowry, and L. E. Smith

- Prediction of the Long-Term Stability of Polyester-Based Recording Media; D. W. Brown, R. E. Lowry, and L. E. Smith.

- Examination of Processes in Thermogravimetry; B. Dickens

- Thermogravimetric Studies of Polymer Degradation; B. Dickens . . . . . . . . . . . 39

- Variable Heating Rate Thermogravimetry; J. H. Flynn . . . . . . . . . . . . . . . . 39

- Photolytic and Thermal Degradation of Poly(Methyl Methacrylate); B. Dickens . . . . 39 
- Fluorescence Measurement of Antioxidant Migration from Low Density Polyethylene

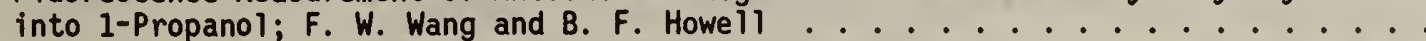

- Diffusion of Small Molecules in Polymers; S. S. Chang, J. R. Maurey, and W. J. Pummer

- Solubilities of $n$-Alkanes in Polyolefins and in Various Solvents; S. S. Chang and J. R. Maurey . . . . . . . . . . . . . . . . . . . . . . . .

- Measurements of Relative Diffusion Constants in Polymers; G. A. Senich . . . . . . 42

- Measurement of Antioxidant Diffusion by Microfluorimetry; F. W. Wang and B. F. Howell ............................

- Measurement of Translational Diffusion of Polymers by Fluorescence Photobleaching Recovery Technique; F. W. Wang and W. H. Grant . . . . . . . . . . . . . .

- Partition Coefficient of Vinyl Chloride in Poly(vinyl Chloride); S. S. Chang and W. J. Pummer

- Self-Diffusion in n-alkane Crystals; B. L. Farmer and R. K. Eby . . . . . . . . . 45

VI. MICROSTRUCTURE AND PERFORMANCE OF DIELECTRIC PLASTICS . . . . . . . . . . . . . . . 46

\section{G. T. DAVIS - TASK LEADER}

- Automated Dielectric Measurement; F. I. Mopsik . . . . . . . . . . . . . . . 46

- Dielectric Loss Spectra of Polymers in the Microwave Region; A. J. Bur . . . . . . 47

- Resistivity Measurements on Ultra-Drawn Poly(vinylidene fluoride) (PVDF) Films; A. J. Bur . . . . . . . . . . . . . . . . . . . . . . . . . .

- Conducting Polymers; H. P. R. Frederikse, C. K. Chiang, and W. R. Hosler .....

- Structure and Performance of Piezoelectric Polymers; G. T. Davis, M. G. Broadhurst, J. Lovinger, and T. Furukawa ... . . . . . . . . . . . . . . . . . .

- Polarization and Space Charge Studies; A. S. DeReggi, F. I. Mopsik, and A. J. Bur ... . . . . . . . . . . . . . . . . . . . . . . . .

- Spontaneous Polarization in Ferroelectric Polymers; A. S. DeReggi, S. C. Roth, G. T. Davis, and M. G. Broadhurst.........................

- Fabrication of Thick Piezoelectric Polymer Film; A. J. Bur, M. G. Broadhurst, and A. K. Tsao . . . . . . . . . . . . . . . . . . . . . .

- Acoustic Sensors; A. S. DeReggi and S. C. Roth . . . . . . . . . . . . . . . 51

- Fast Ion Transport in Solid State Polymers; C. K. Chiang, G. T. Davis, A. J. Bur, and A. S. DeReggi . . . . . . . . . . . . . . . . . . . . . .

- Evaluation of Cellulose Acetate as a Neutron Dosimeter; M. G. Broadhurst, A. J. Bur, and R. B. Schwartz.

VII. TESTS, STANDARDS AND CHARACTERIZATION FOR MANUFACTURING AND DURABILITY OF DENTAL AND MEDICAL MATERIALS

\section{J. M. CASSEL - TASK LEADER}

- Dental Composite Chemistry and Development; G. M. Brauer, J. M. Antonucci, J. W. Stansbury, J. M. Cassel, S. Venz, and R. L. Bowen ..................

- Improved Bonding Techniques and New Dental Cements; G. M. Brauer, R. L. Bowen, J. M. Antonucci, J. W. Stansbury, D. W. Misra, E. Cobbs, and J. M. Cassel .....

- Wear Mechanisms, Wear Testing and Improving Durability in Dental Composite Materials; W. Wu, J. E. McKinney, E. E. Toth, and J. M. Cassel .......... 
- Composite Restorative Materials; J. M. Antonucci, G. M. Brauer, S. Venz, N. W. Rupp, and J. M. Cassel

- Porcelain Fused to Base Metal Alloys and Dental Ceramics; J. A. Tesk, R. W. Hinman, W. deRijk, D. L. Menis, G. Widera, J. Sanecki, O. Frankiln, and

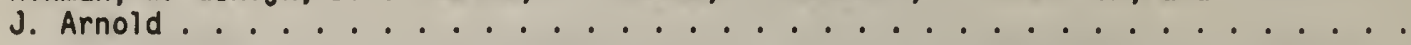

- Dental Casting Alloys and Investments; J. A. Tesk, R. M. Waterstrat, R. W. Hinman, W. deRijk, and D. L. Menis

- Dental Chemistry and Preventive Dental Treatments; W. E. Brown, L. C. Chow, M. Mathew, M. S. Tung, G. L. Voge 1, S. Takagi, T. M. Gregory, and A. Uchida . . . .

- Clinical \& Laboratory Investigation of Dental Amalgams, Composites \& Cements; N. W. Rupp, G. C. Paffenbarger, and R. M. Waterstrat . . . . . . . . . 60

- Adhesion of Synthetic Polymers on Solid Surfaces; R. E. Dehi and W. H. Grant . . . 61

- Characterization of Porosity of Porous Implant Materials; R. E. Dehl . . . . . 61

- Surgical Bone Cement Modification; J. A. Tesk, G. M. Brauer, W. Wu, and J. E.

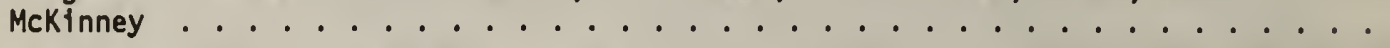

- Dental and Medical Materials Standards; J. M. Cassel, J. A. Tesk, G. M. Brauer, and

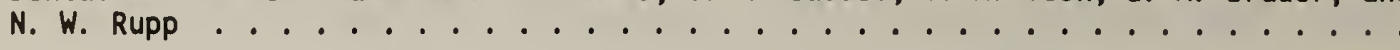

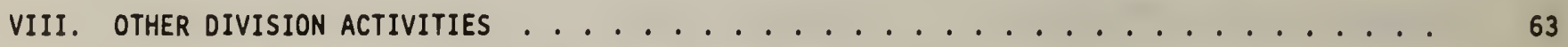

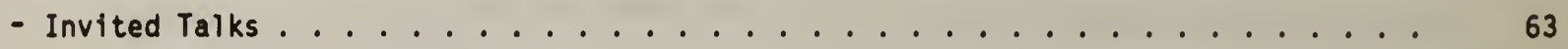

- Seminars for Staff and Guests . . . . . . . . . . . . . . . . . . 76

- Technical and Professional Committee Participation and Leadership . . . . . . . . 79

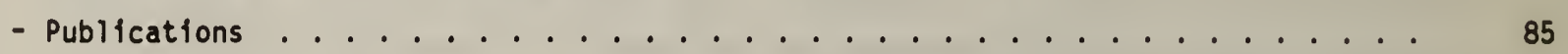

- Sponsored Conferences . . . . . . . . . . . . . . . . . . . . . 91

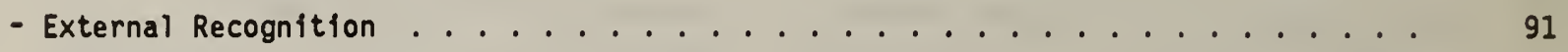

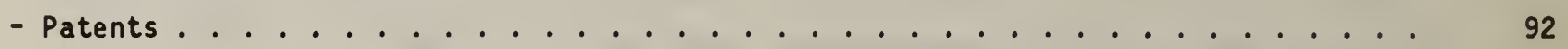


Ronald K. Eby, Chief

Martin G. Broadhurst, Deputy Chief

S. M. Thomas, Administrative Officer

Barbara A. Hyde, Secretary

Although synthetic polymers have been used as materials for technology during only three quarters of a century, they have left little of our economy, technology, industry, science, and culture untouched. We have moved rapidly into an age in which an evergrowing number of humanity's needs are served by polymers. The volume currently produced exceeds that of steel and forms the basis of industries which ship. over $\$ 240$ billion of goods; add over $\$ 106$ billion of value by manufacture (a measure of the relative economic importance of manufacturing among industries) and provide 3.3 million jobs. Recent summaries show that polymers and polymer composites research al ready accounts for about 47 percent of the total industrial R\&D expenditure for metals, polymers, and inorganic materials. Among these materials, polymers also constitute about 40 percent of the value added by manufacturer, 45 percent of the jobs, 49 percent of the number of scientific publications, and 42 percent of the American Society for Testing and Materials (ASTM) standards.

Polymers have been shaped by intellectually sophisticated research which has been recognized by a number of Nobel Prizes. The resulting materials have contributed to national industrial productivity through their economy, ease of fabrication, and usefur properties such as low density, corrosion resistance, and toughness. Polymers are energy efficient; the largest volume polymer, polyethylene, uses 29 percent of the energy per unit volume required by steel for conversion from raw to refined material and yields further savings in processing and shipping. In fact, studies have shown that for every barrel of oil used to make plastic products, approximately one barrel is saved in comparison with the use of the next best alternative material. These useful properties have enabled polymers to contribute to increased national economic strength both by creating entirely new products and by substituting for other materials in existing products.

Polymers have entered a widening range of increasingly sophisticated and demanding applications. Polyvinylidene fluoride films are used as piezoelectric transducers in high fidelity sound systems. Structural parts of aircraft are constructed from composites with a polymer matrix and sometimes a polymer reinforcement. Polyurethane elastomers are used to pump blood in circulatory assist devices. The list of such examples is very long, and includes trends for the increased use of polymers in packaging, construction, transportation, national security, electronics, information handling, clothing, electrical appliances, energy, dentistry and medicine. Since polymer science and technology is on the steep part of the learning curve, these trends create many new needs for scientific concepts, measurements, standards, and data that can be used to ensure their reliable performance in the use, processing, and other phases of the material cycle. The programs of the Division are aimed at the fundamental scientific basis of these needs.

To identify the key needs with the best prospects for payoff, the managers of the Polymer Science and Standards Division make structured visits to industrial and Government laboratories. Visited sites include Be11 Laboratories, E. I. DuPont de Nemours, Inc., 3 M, Army R\&D Commarid, General Motors Corporation, Boeing, and Lawrence Livermore Laboratories. Surveys have been conducted in the areas of durability, composites, and processing. These and other contacts with research associates, experts in industry, trade associations, standards associations, guest workers, Government, and academia ensure that the Division's efforts are directed toward fostering the effective use of polymers in solving generic national problems. These include the growth of the economy, national security, efficient Government, materials utilization, and conservation of critical materials.

Current activities are associated into areas concerned with long-term research on the scientific basis for mechanical reliability in load-bearing applications, chemical durability, lifetime prediction, migration of protective additives, characterization methods, standards for molecular composition and performance properties, dielectric properties, processing and reliability of composites, and reliable dental and medical materials. Substantial cooperative efforts are conducted with research associates from a number of industries, universities, the Department of Defense, the National Institutes of Health, the American Dental Association (ADA), the Food and Drug Administration, and the Bureau of Engraving and Printing. All these efforts lead the staff of the Division into an examination of the scientific basis of those properties which are of concern to people who use, design, and produce useful polymer objects. This work helps society capture, in safe and satisfying ways, the benefits of the increasingly widespread application of polymers.

\section{SOME FY82 ACCOMPLISHMENTS}

\section{POLYMER STANDARDS FOR PROCESS CONTROL AND EQUITY}

- Construction of a selfcalibrating gel permeation chromatograph (GPC) has been completed. A light scattering detector for the GPC measures, in real time, the scattering intensity of the effluent as a function of scattering angle. This allows immediate identification of 
difficulties such as molecular association, microgel formation, etc. which affect the validity of the molecular weight determinations. This instrument will be utilized for the more rapid characterization of new polymer molecular weight standards.

- A high performance liquid size exclusion chromatograph laboratory has been established for the determination of the molecular weight and molecular weight distribution of polymer samples. The apparatus has been automated and may be operated at temperatures up to $150^{\circ} \mathrm{C}$, the temperature required for the dissolution of polyethylene and certain other crystalline polymers. Graphics and database capabilities are built into the software to allow the user easy interpretation of the data once it is collected.

- The certification of Standard Reference Material 1470 for transmission of helium, carbon dioxide, oxygen, and nitrogen gases has been completed. Gas transport properties of polymeric materials are the major determinant of suitability for end use in a great variety of applications ranging from food and beverage packaging, industrial gas separation processes, and sterilization of medical devices, to substrates and packages for semiconductor products. Gas molecules are useful as probes of materials properties at the microstructural level.

- Polymer properties can be greatly altered by controlling the size and shape of the phase separated domains of polymer blends and copolymers. In collaboration with $\mathrm{Dr}$. H. Hasegawa of Kyoto University, we have prepared model systems of polystyrene-polyisoprene which have regular lamellar domains. Dimensions of the deuterium labeled polystyrene block of this diblock copolymer and the dispersed homopolystyrene molecules have been measured at different directions relative to the domain orientations by small angle neutron scattering (SANS). Work on the characterization of blends by SANS is being carried out in collaboration with a Research Associate from Exxon.

- A new statistical mechanical model for a chain liquid has been developed which shows a solid-liquid transition as well as a liquid-vapor transition. This work is being carried out in collaboration with R. H. Lacombe of IBM (Fishkill). This model may prove to be useful in describing the phase behavior of polar polymer blends.

- A new instrument for forced Rayleigh light scattering is being completed. A split laser beam is recombined at a small crossing angle on a sample which includes a trace of polymer labeled with a photochromic dye. This beam is pulsed to produce an interference pattern which bleaches a holographic grating in the sample. A second laser beam is diffracted by the grating. As the labeled polymers diffuse, the grating is destroyed. Thus, measuring the intensity decay of the diffracted beam yields the diffusion coefficient of the labeled species. Since the distance scale is typically several orders of magnitude smaller than macroscopic tracer methods, it is possible to measure extremely small diffusion coefficients such as those expected for polymer molecules in concentrated solutions and melts.

\section{MECHANICAL DURABILITY OF POLYMERS}

- Carbonyl concentrations have been determined by FTIR for fatigued ultra high molecular weight polyethylene as a function of number of cycles, frequency and peak stress level. The results suggest that mechanical failure occurs at a particular concentration of carbonyls. The measurement of carbonyl concentration may form the basis of an NDE method.

- Carbon -13 NMR techniques have been developed to elucidate molecular structure of ultra high modulus polymeric materials. The fraction of material in the form of molecules that bridge one crystalline lamella to another has been determined. Bridge molecules are thought to be responsible for the high tensile properties and the quantitative NMR determinations can be used to evaluate the effects of processing conditions on the molecular arrangements. This technique is being applied to high strength poly(ethylene terephthalate) fibers in a joint project with the Firestone Tire \& Rubber Co.

- A new improved test method for environmental stress crack resistance of ethylene-based plastics has been developed. The method has the advantages of a reduction in the time required to carry out the test, and a reduction of experimental scatter in the data. A description of the test method and a summary of test results, including the appropriate statistical data, has been presented to Committee D-20.12.03 of the ASTM.

- A viscoelastic relationship has been developed that is capable of describing quantitatively both creep and recovery behavior of polymeric materials which have not been mechanically preconditioned. Previous theories could be applied successfully only to materials that were first mechanically conditioned and thus the mechanical behavior of as-processed materials could not be adequately predicted. 
The creep and stress relaxation behavior of ultra high molecular weight polyethylenes used in synthetic implant applications has been determined as a function of molecular weight and various thermal treatments. Electron microscopic investigations of deformed specimens have shown that preferential deformation occurs at boundaries which delineate the interfaces between the sintered grains of raw polymer.

- A torsional dilatometer has been designed and constructed to determine the effects of torsion on the volume of polymer glasses. The data will serve to test theories concerning the behavior of polymer glasses under deformation and the effects of deformation on the aging of these materials.

\section{PROCESSING AND RELIABILITY OF POLYMER COMPOSITES}

- A failure model has been developed and applied to elastomer modified epoxy formulations. The temperature dependence of the fracture energy could be described with a single Arrhenius type activation energy whose value was identical to that obtained from yield experiments. This provides the first direct evidence that yielding is a crucial step in the toughening mechanism of elastomer modified epoxy resins systems used in aerospace applications.

- A joint program involving NASA, Hercules, Hexcel, the University of Illinois, and NBS is addressing the need for test procedures to assess the susceptibility of polymer composites to delamination. Delamination is a major concern in the mechanical reliability of continuousfiber reinforced composites. Results during the past year have led to guidelines for the design of test specimens in terms of size, geometry, and lay-up (the sequence of fiber layers and their orientation).

- A research associate from Martin Marietta Corporation, spent six months at NBS applying Fourier transform infrared spectroscopy (FTIR) to characterization of the processing of composites used in the Space Shuttle Program. Results indicated that IR spectroscopic data could be used to improve the processibility of composites.

- A model has been developed to analyze small angle $x$-ray data of particulate composites in terms of three phases: the polymer matrix, the particulate reinforcement, and microcracks or voids. From this model the content and characteristic lengths of microcracks or voids can be determined from the angular dependence of the x-ray scattering intensities. The method provides a means of assessing the state of damage of particulate-reinforced composites.

- An ultrasonic test method has been developed to study the curing process (the liquid to solid conversion) in thin layer films. The method will provide a better understanding of the chemistry of processing thermoset polymers and more reliable mechanical properties data of polymer matrix materials.

- A spectroscopic technique has been developed to monitor the chemical reactions that occur during processing polymer composites and subsequent to processing (post-cure). Using this technique the effects of initiator type and concentration as well as the molecular flexibility of the prepolymers have been investigated. The degree of post-curing was found to be affected primarily by the molecular flexibility of the prepolymer.

\section{ADDITIVE MIGRATION AND CHEMICAL DURABILITY OF POLYMERS}

- Kinetic data on the production of acidic groups in the hydrolysis of crosslinked polyester polyurethanes and polyethylene terephthalate have been measured. These data are being used in kinetic schemes recently developed at NBS to predict the lifetimes of these polymers when used as base materials and magnetic tape binders to store archival information. The information provides far more realistic estimates of when archival material should be recopied.

- Thermal degradation occurs during the processing stage for many polymer-based materials. Activation energies and a kinetic scheme for thermal degradation have been provided for polyisobutylene using a thermogravimetric technique deveoped at NBS. The important effect of pressure on volatile production and on the measured kinetic values used to estimate the extent of thermal degradation has been demonstrated by means of calculation and experimental data. New thermogravimetric techniques have been developed which greatly ease the characterization of degradation mechanisms.

The relative contributions of thermal and photolytic degradation in PMMA used as solar cover plate materials are being elucidated by first exposing the materials to controlled environments and then determining the molecular weight as a function of depth in a series of highly precise measurements. The contributions of other factors to the degradation will be examined in a continuing series of experiments. The aim is to predict molecular weight changes as a function of exposure. This will make it possible to estimate changes in mechanical properties with exposure. 
Highly sensitive fluorescence techniques have been developed to provide measurements on migration rates, and have been applied to: (1) measurement of the migration rate of an amine antioxidant from low density polyethylene, and (2) translation diffusion of chain molecules in semi-dilute solution. The second area provides data needed for tests of the scaling and reptation concepts proposed by de Gennes.

- An inverse phase gas chromatographic technique has been developed and applied to the measurement of diffusivities of several migrants in high density polyethylene. Such data are used in modelling the rate of loss of plasticizers and stabilizers from polymeric materials.

\section{MICROSTRUCTURE AND PERFORMANCE OF DIELECTRIC PLASTICS}

- A new time domain dielectric spectrometer has been developed. It rapidly measures and transforms charge-time data to dielectric constant and loss as a function of frequency over the range from $10^{-3}$ to $10^{5} \mathrm{~Hz}$. This measurement technique, which meets an industrial need for rapid and accurate measurement over a wide frequency range, will be adapted for industrial use by a Research Associate from G.E.

- A major review of data and mechanisms for dielectric loss in materials at microwave frequencies was prepared for the U.S. Army Civil Engineering Research Laboratory. This data is needed to better understand and control the reflection of microwaves from solid structure.

- A ferroelectric to paraelectric transition in a copolymer of vinylidene fluoride and trifluoroethylene was shown to be due to a change in conformation of the chain within the crystal phase of the polymer. Such conformational changes have important implications on the long-term, high temperature durability of piezoelectric polymeric transducers. This work was done in a cooperative project with Bell Laboratories.

- The NBS-perfected technique for measuring space charge and polarization distribution in a thin polymer film has been successfully applied to electron-beam charged PVC and to PVDF after reversal of the poling field. Polymer-charge interactions leading to nonuniform electric fields are believed to play a key role in the performance of insulating materials.

- The use of polyvinylidene fluoride piezoelectric and pyroelectric transducers at elevated temperatures is limited to about $100{ }^{\circ} \mathrm{C}$ due to loss of polarization although the melting point of the polymer is near $170{ }^{\circ} \mathrm{C}$. A rapid method for measuring pyroelectric response as a function of temperature has been developed as a first step in determining the variables which influence this loss in order to provide the basis for improved high temperature performance. This work was done in collaboration with the Brown Research Foundation.

- The processing conditions required to produce thick films of polyvinylidene fluoride transducers for sensitive hydrophones were successfully demonstrated to the Naval Ocean Systems Command. These thick films are most promising for hydrophone applications.

- Piezoelectric polymer sensors produced at NBS are being evaluated as monitors in cardiac patients in collaboration with the CNR Institute of Clinical Physiology.

- With support from the Office of Naval Research, NBS has initiated a long-range investigation into the relation between morphology and structure of polymers and the highiy specific property of fast ion transport. These types of polymers have potentially high payoff applications as solid state electrolytes in batteries and fuel cells and as highly specific electrochemical sensors.

- At the request of the Department of Energy, an NBS evaluation has shown that electrical resistivity changes in cellulose acetate after neutron irradiation would not be useful as an effective neutron dosimeter. This conclusion is contrary to a report published by another laboratory.

TESTS, STANDARDS, AND CHARACTERIZATION FOR MANUFACTURING AND DURABILITY OF DENTAL AND MEDICAL MATERIALS

- A silver staining technique developed for investigating wear damage in dental composites was applied to assessment of microleakage and composite restorations and increased precision relative to a more laborious radioisotope $\left(\mathrm{Ca}^{45}\right)$ technique was demonstrated.

- A promising new monomer type, polythiols, was introduced to the dental materials research community. A high molecular weight, moderately viscous polythiol, pentaerythritol tetra (3-mercaptopropionate), has promise for reducing residual unsaturation and polymerization shrinkage in dental composites. 
Developed a procedure employing coupling agents and a mineralization step that achieves a degree of stable bonding to dentin approximately three times that hitherto reported. As a result, the potential exists for improved clinical treatment of erosion, root caries and other conditions by reducing the amount of dentin that needs to be cut for mechanical retention.

Several new dental cement innovations were introduced to the dental materials research community. Arousing particular dental profession and industry interest is a cement formulation based on hexyl vanillate, ethoxybenzoic acid and zinc oxide. Low water sorption, high strength, non-inhibiting to free radical polymerization of subsequently applied composites or resins and predictably good tissue tolerance are important characteristics of this new cement.

Silver staining and small angle x-ray scattering analysis of removed clinical composite restorations have been used to define mechanisms of wear. In the laboratory a softening of the composite resin matrix by a range of food-simulating solvent media has been shown to produce similarly damaged materials. This information has permitted appropriate modification of in vitro testing and resulted in emphasizing the crucial importance of the degree of cure in the composite polymer.

- Fourier Transform Infrared has been used to demonstrate the extent of under cure of dental composite resin matrices achieved at oral temperatures relative to that reached at higher temperatures. A higher degree of cure is obtained by building more polymer backbone flexibility into the monomer formulation indicating a viscosity controlled process. These results provide important guides for laboratory testing and developmental directions of the dental industry.

- Research pin-pointed food debris and oral liquid interactive softening of dental composite restorations as initial steps in clinical. loss of restoration material. Based on these results, an erosion (high velocity liquid jet) test was designed and assembled. SEM examination of composite surfaces exposed to the test revealed surface deterioration similar to that experienced clinically. The test in conjunction with other wear test procedures is expected to provide industry and other research groups with an important assist in development of more durable composite restorations.

- A new concept for evaluation of porcelain-metal bond strengths has been developed. The test uses four-point bending of a metal strip to which porcelain is fused and measures a tensile strength without influence from shear stresses. Three dental manufacturers have sent personnel to develop a first hand experience with the test procedure which is anticipated to be useful in industrial examinations of porcelain-alloy combinations and as a specification test method.

- Amines have been shown in this laboratory to be more reactive with the resin, BIS-GMA, than current commercial materials. They have now also been shown to be useful for upgrading surgical bone cement. These amines in conjunction with a substitution of dicyclopentyloxyethyl methacrylate for the presently used methyl methacrylate give a faster cure with less liberation of heat.

Characterization of the porosity in low modulus porous polymeric materials designed for medical and dental implant purposes is important for establishing a correlation of porosity and the implant stabilizing tissue or bone ingrowth. Mercury intrusion, BET surface area and quantitative stereology measurements have been made. The nature of the porosity characteristics as determined by each technique is being defined.

In the following pages, the activities of the Division are presented formally in six tasks, each described separately:

POLYMER STANDARDS FOR PROCESS CONTROL AND EQUITY

MECHANICAL DURABILITY OF POLYMERS

PROCESSING AND RELIABILITY OF POLYMER COMPOSITES

ADDITIVE MIGRATION AND CHEMICAL DURABILITY OF POLYMERS

MICROSTRUCTURE AND PERFORMANCE OF DIELECTRIC PLASTICS.

TESTS, STANDARDS, AND CHARACTERIZATION FOR MANUFACTURING AND DURABILITY OF DENTAL AND MEDICAL MATERIALS 


\title{
I. C. Sanchez
}

\section{Task Leader}

This task provides standard reference materials (SRMs) to the $\$ 30 \mathrm{~B} /$ year synthetic polymers producing industry. Molecular weight SRMs are used extensively in industry to calibrate gel permeation chromatographs (GPCs) which are used routinely to measure polymer molecular weights and molecular weight distributions. The latter are the most important physical feature in determining a polymer's processability and its ultimate performance. Gas transmission and rubber SRMs are used in process control applications.

Improved characterization techniques are essential to providing improved SRMs. A fundamental knowledge of polymer solution properties such as osmotic pressure, viscosity, diffusional behavior, and scattering power is essential to this goal. As a consequence, a majority of the activities in this task involve experimental and theoretical studies of polymer solution properties. As a spinoff of our characterization activities, this task also provides data and concepts which are useful in polymer processing.

The execution of this task was assisted by $\mathrm{Or}$. D. Lohse, an industrial research associate from Exxon, Dr. J. G. Dillon, a guest worker from the Food and Drug Administration, and A. Z. Akcasu, a guest worker from the University of Wisconsin.

\section{Self-Calibrating Gel Permeation Chromatography}

\section{P. H. Verdier}

Gel permeating chromatography is a widely accepted technique for estimating the molecular weight distribution (MWO) of high polymers. However, the usefulness of the conventional GPC apparatus is limited by the need to provide calibrants for each polymer measured of known molecular weight over the entire molecular welght range in which the MWD is significantly different from zero. The calibration depends, among other things, upon the chemical nature, degree of branching, etc., of the polymeric material so that each new material requires a fresh calibration. The so-called "universal calibration" hypothesis, while useful, is limited to comparisons of polymers of similar shape and, in any event, is inadequate for quantitative determinations. Some instruments, one of which is commercially avallable, attempt to circumvent the need for calibrants by adding a single-angle light scattering detector to the usual concentration-sensitive detector. However, this does not allow the extrapolation to zero scattering angle which is required, in principle, to relate scattering intensity to molecular weight. In addition, qualitative information on the variation of scattering with angle, normally required to give assurance that meaningful results are being obtained, is not available.

We are designing and constructing a light scattering detector for the GPC which measures. in real time, the scattering intensity as a function of scattering angle and which is controlled by a dedicated minicomputer in a way that allows scattering to be measured as a function of scattering angle and concentration. The instrument will allow continuous monitoring of the variation of scattering with angle. This will allow immediate identification of difficulties such as association, microgel formation, etc., which could affect the validity of the molecular weight obtained, an important consideration for work on new and unstudied materials. In addition to molecular weight, the mean-square radius (radius of gyration) will be obtained as a function of molecular weight, at least in the higher ranges of molecular weight, providing useful information for the characterization of branched polymers.

We expect to complete construction of the instrument by the end of FY82. In the coming year, we will start making measurements with it and developing procedures for high-quality molecular weight determinations.

\section{Liquid Size Exclusion Chromatography}

\author{
H. L. Wagner and J. D. Barnes
}

A commercial high performance liquid size exclusion chromatograph has been installed for the determination of the molecular weight and molecular weight distribution of polymer samples. The apparatus has been automated and may be operated at temperatures up to $150{ }^{\circ} \mathrm{C}$, the temperature required for the dissolution of polyethylene and certain other crystalline polymers. The unit is self-contained so that as many as 16 samples may be prepared internally. The conditions for each sample may be programmed separately and as many as nine replicate injections are possible. In conjunction with the automated data acquisition system, it is possible to run for over a 24 hour period without operator attention. The actual time required will depend on the columns and time allowed between runs for sample preparation and data acquisition. 
The software has been designed so that the user does not need to learn the operating system of the computer and so that there is a one-to-one correspondence between the data required to program the chromatograph (which is microprocessor controlled) and that required to perform data analyses in the computer. Graphics and database capabilities are built into the software to allow the user easy interpretation of the data once it is collected.

This facility will be employed for many of the problems encountered in the Polymer Science and Standards Division where molecular weight distribution is a controlling variable. Changes in molecular weight distribution have been found to correlate with changes in many physical properties such as tensile strength, modulus, relaxation times, elongation, and stress cracking. The equipment has already been employed successfully in support of a project for the NBS Center for Building Technology involving the affect of ultraviolet exposure on polymethyl methacrylate.

\section{Limiting Viscosity Number of Ultrahigh Molecular Weight Polyethylene}

\section{H. L. Wagner and J. G. Dillon}

Limiting viscosity number (intrinsic viscosity) measurements are required for the specification and control of ultrahigh molecular weight polyethylene (UHMWPE), one of the most extensively employed surgical implant materials. Because the viscosity number is dependent on shear rate, it should be measured at zero or very low shear rates where this dependency does not occur. For this purpose a low shear rate viscometer capable of being operated at high temperatures ( 135 to $145^{\circ} \mathrm{C}$ ) was constructed. The problems associated with the viscometer and some of the measurement difficulties, described in previous reports, have been overcome.

This type of viscometer is not commercially available and requires more than routine skill for its operation. However capillary viscometers, in which the shear rate can be quite high, have been successfully employed for many years for lower molecular weight polymers which are not sensitive to shear rate. A set of experiments were performed in which the same solutions, fractions of UHMWPE, were measured in decalin at $135{ }^{\circ} \mathrm{C}$ in both the low shear viscometer and a commercial capillary viscometer which is of a standard design. As shown in figure 1 an empirical relation exists between the two, so that a routine capillary viscometer measurement may be used to estimate the low shear value provided the capillary viscometer is of the same capillary size and volume as used in these experiments.

The measurements were made on fractions of UHMWPE obtained by the method described below. In order to assure complete dissolution of these fractions it was necessary to cut the material into $1 / 2 \mathrm{~mm}$ bits and to heat in decalin at $180^{\circ} \mathrm{C}$ under nitrogen for one hour, with occasional gentle mixing to prevent agglomeration of the bits. This method was successful with all but the highest molecular weight fraction. The poor reproducibility of the results for this fraction, where viscosity numbers were of the order of $60 \mathrm{dl} / \mathrm{g}$, indicates that solution was incomplete. From 4 to 8 measurements were made for each of 10 fractions to determine the limiting viscosity number of each fraction. These ranged from 9 to $52 \mathrm{dl} / \mathrm{g}$.

These or similar fractions will be used to obtain the relationship between molecular weight and viscosity numbers by light scattering for these very high molecular weight polyethylenes. The Mark Houwink relation between viscosity and molecular weight, obtained empirically for lower molecular weight polymers, is not expected to hold for UHMWPE. When this relation is available, it should be possible to obtain molecular weights from simple capillary viscosity measurements in a standard viscometer.

\section{Fractionation of Ultrahigh Molecular Weight Polyethylene}

\section{H. L. Wagner}

The fractions described above were obtained by crystallization from a 1 percent solution of UHMWPE in decalin. This solution was cooled slowly from $135{ }^{\circ} \mathrm{C}$ with rapid stirring until polymer appeared on the stirrer. This fraction was removed and the process was repeated until the next fraction appeared. Ten fractions were obtained by this method. About 12 percent of the material did not precipitate by the stirring process and was recovered as a residue when the solution was cooled. Limiting viscosity numbers ranged from $9 \mathrm{dl} / \mathrm{g}$ to $50 \mathrm{dl} / \mathrm{g}$ in decalin at $135{ }^{\circ} \mathrm{C}$. Employing the conventional Mark Houwink relationship for lower molecular weight polymers this corresponds to a range of from about 900,000 to 10 million in molecular weight. The viscosity average molecular weight for the residue, if calculated in this way, is about 700,000 and would be expected to be very broad in distribution. This was confirmed in a preliminary GPC run which indicated the presence of material below 10,000 , as well as some above 1 million. 


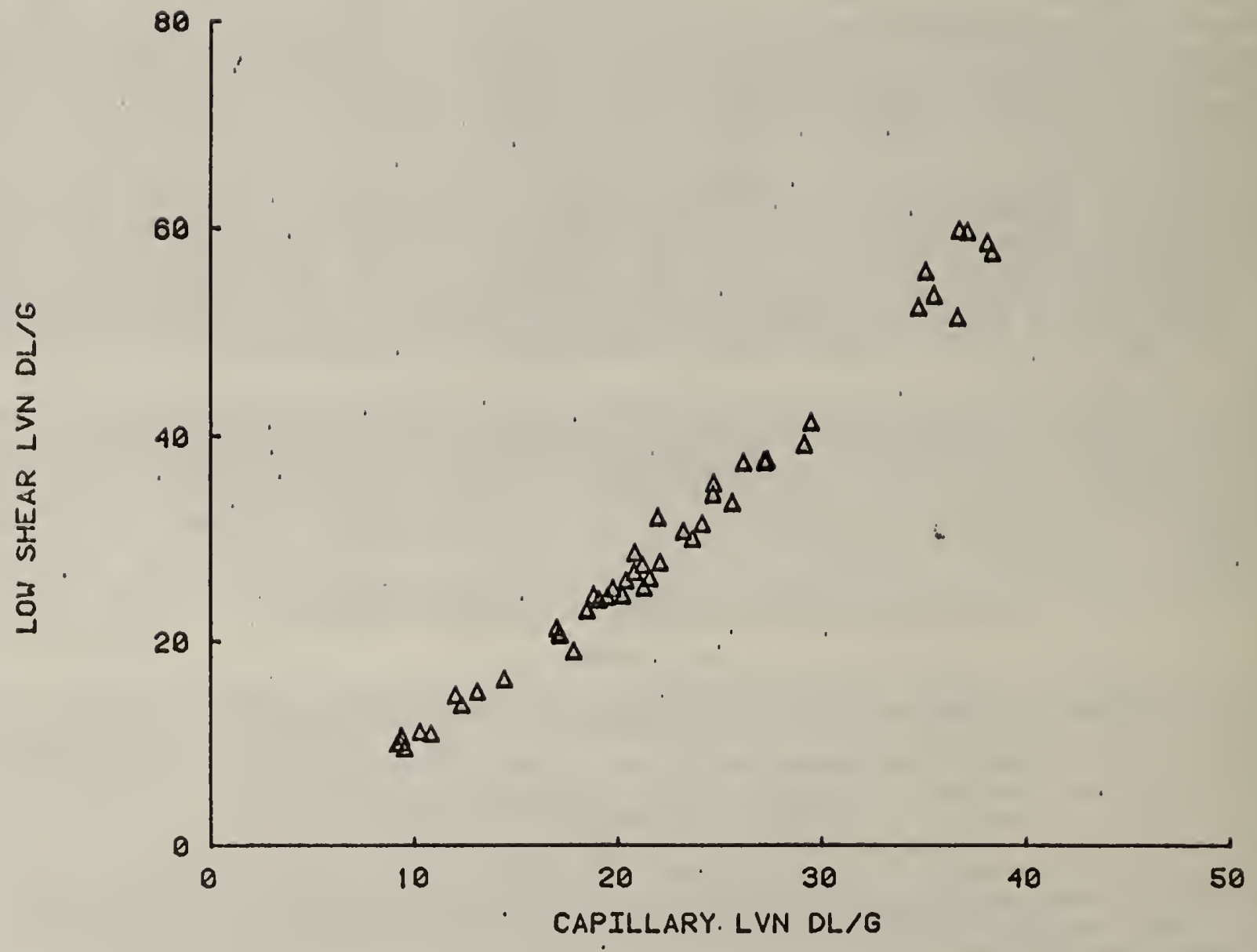

Figure 1. Relation between LVN at zero shear and LVN measured in a Capillary Viscometer. 


\section{F. W. Wang and F. L. McCrackin}

A new method for the analysis of ultracentrifugation interference patterns with the use of a commercial image digitizer is given. The application of the method to the sedimentation equilibrium data for SRM 1478 Polystyrene leads to a weight-average molecular weight of $37,400 \mathrm{~g} / \mathrm{mol} \mathrm{having}$ a sample standard deviation of 0.7 percent and expected systematic error 1 imit of 2 percent.

\section{Standards for Gas Transmission Measurements}

\section{J. D. Barnes}

Gas transport properties of polymeric materials are the major determinant of suitability for end use in a great variety of applications ranging from food and beverage packaging, industrial gas separation processes, and sterilization of medical devices, to substrates and packages for semiconductor products. Gas molecules are useful as probes of materials properties at the microstructural level. The measurement system for gas transport properties has suffered from a lack of reliable standards.

NBS is addressing these needs by developing improved measurement technology and by characterizing polymeric materials for use as Standard Reference Materials. The certification of SRM 1470 for its gas transmission properties with respect to helium, carbon dioxide, oxygen, and nitrogen has been completed. It is expected that this material will now be useful to a wider variety of users.

The recertification experiments produced approximately 720 values of the permeance and 540 values of the time-lag (the time-lag for helium was too short to measure). The results can be summarized in the form:

$$
\hat{Q}(T)=\hat{Q}(23) \times \exp \left(\beta_{Q}[T-23]\right) \text {, }
$$

where the "^" denotes that the quantity is an estimated value as derived from fitting to the experimental data. " $Q$ " represents either the permeance, $P$, or the time-lag, $\tau$, as appropriate and $T$ is the temperature of measurement in ${ }^{\circ} \mathrm{C}$. Values of $\hat{Q}(23)$ and $\boldsymbol{\beta}_{Q}$ obtained from the analysis of the permeance and the time-lag data are given in tables 1 and 2 respectively. Since the time-lag for carbon dioxide depends upon the upstream pressure it is necessary to multiply the estimates obtained from equation 1 by a term of the form:

$$
\exp \left[a_{1}\left(p_{u}-101.32\right)+a_{2}\left(p_{u}-101.32\right)^{2}\right]
$$

where the upstream pressure, $P_{u}$, is expressed in $\mathrm{kPa}$. Estimates derived from equations (1) and (2) are valid only for pressures between 67.5 and $135 \mathrm{kPa}$ and for temperatures between 18 and $31{ }^{\circ} \mathrm{C}$, the ranges covered in our experiments.

Tables 1 and 2 provide additional statistical data that can be used to qualify the estimates derived from the fitting process. $c_{\mu}$ is the coefficient of variation of the expected value, its numerical value largely determined by the sampling error arising from the selection of test specimens. $C_{s}$, the coefficient of variation of the "specimen effects," is a measure of the inhomogeneity of the lot of SRM material. $C_{r}$, the coefficient of variation of the residuals from the fit, is a measure of the extent to which individual data values depart from the model in equation (1). We have chosen not to construct the usual confidence or tolerance intervals because we do not have enough data on the distribution of the specimen effects. We are studying additional specimens to better define the structure and distribution of the specimen effects.

We are also seeking simple auxiliary measurements such as the specimen thickness or the density that can be used to define correction factors for reducing the measured values to a common basis. In the absence of such a scheme the specimen-to-specimen variability is the dominant factor limiting the precision that can be expected when comparing measurements among different stations in a measurement system.

The observed decrease of the time-lag for carbon dioxide with increasing pressure is consistent with published data that have been interpreted using a "partial immobilization" model. It is somewhat surprising that we do not observe a concommitant decrease in the permeance; perhaps the pressures used are too low or the pressure range is not broad enough.

The effects of partial immobilization are small in the range of conditions covered in our experiments. Users who wish to carry out measurements at much higher pressures and temperatures should realize that they may have to take explicit account of this phenomenon. 
We are modifying our apparatus to operate at higher pressures and at temperatures encompassing the glass transition of poly(ethylene terephthalate) (PET) in order to better characterize the physics of partial immoblization and its relationship to the glass transition.

We are working to identify needs for materials with transmission rates that are both higher and lower than those of SRM 1470.

Since it is difficult to control processes for making plastic films in order to limit variability in their physical properties to one percent or less, we find that material inhomogeneity is an obstacle to the development of better SRMs. It is also difficult to use such materials as transfer standards because their characteristics often drift with time and test specimens often do not tolerate abuse suffered during the measuring process.

We have modifled the software for our computer-controlled gas transmission measuririg facility so that plotting of the raw data and reduction of the data to permeance and time-lag values are done on-line at the conclusion of each experiment. The software generates databases that can be input directly into statistical analysis programs over an NBS Net TIE.

New data acquisition hardware has allowed us to measure time-lags of less than one second. Further work must be done to detect the presence of instrumental time constants that can interfere with the measurement of the diffusion time-lags of the materials.

Table 1

Fit Results for Permeance Data

\begin{tabular}{lccccc}
\hline & \multicolumn{5}{c}{ Gas } \\
Parameter & $\mathrm{N}_{2}$ & $\mathrm{O}_{2}$ & $\mathrm{CO}_{2}$ & $\mathrm{He}$ & Units \\
\hline$P(23)$ & .0421 & .352 & 1.722 & 13.79 & $\mathrm{pmo} / \mathrm{m}^{2} \cdot \mathrm{s} \cdot \mathrm{Pa}$ \\
$\beta_{P}$ & .0521 & .0376 & .0309 & .0287 & $1 / \mathrm{K}$ \\
$C_{\mu}$ & .023 & .014 & .018 & .012 & \\
$C_{s}$ & .057 & .045 & .056 & .037 & \\
$C_{r}$ & .050 & .017 & .010 & .009 & \\
\hline
\end{tabular}

Table 2

Fit Results for Time-Lag Data

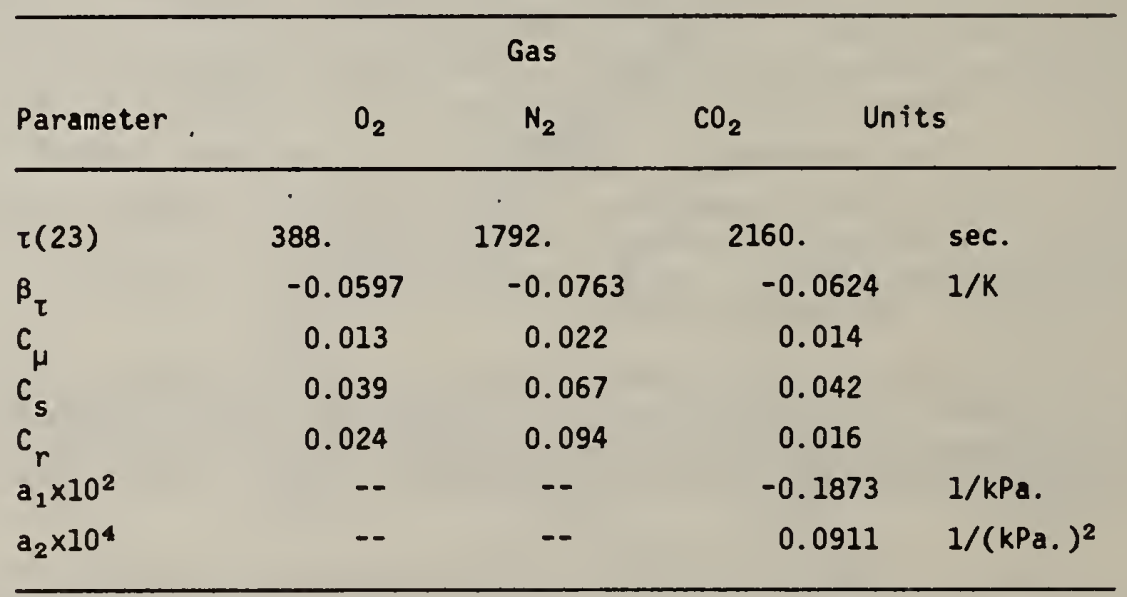




\section{E. J. Amis, C. C. Han, and Y. Matsushita}

The quasielastic light scattering from semidilute solutions of polystyrene in both good or theta solvent conditions has been measured as a function of molecular weight and concentration. We observe two distinct exponential decays separated by several orders of magnitude. The angular dependence of the decay constants is indicative of diffusive processes which we identify with the cooperative

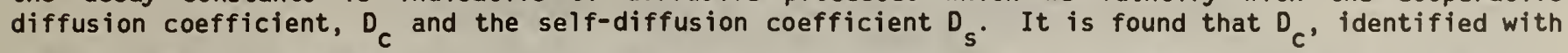
the fast decay, increases with polystyrene concentration and is almost independent of molecular weight. $D_{s}$ on the other hand decreases sharply with concentration and molecular weight. An explanation of the light scattering detection of these two diffusion coefficients can be made which is based only on assumptions inherent in the reptation model of diffusion in semidilute solutions. Over the range of molecular weight and concentration accessible to the technique at the theta condition, measurements of $D_{s}$ are in good agreament with scaling predictions of $D_{s} \alpha M^{2} c^{-3}$. For the good solvent measurements, the agreement with the prediction of $D_{s} \propto M^{2} c^{-1} 75$ is only observed for a narrow window. The blob model gives some explanation for this behavior by allowing that even in a good solvent there is some distance below which excluded volume is unimportant. The chain is Gaussian and the chain shows theta-like $\left(D_{S} \propto c^{-3}\right)$ behavior. The blob model is, however, unable to account for the fact that for $D_{C}$ the agreement with predictions is much better in good solvent than in theta solvent. These experiments point out, once again, that the degree of agreement with scaling predictions is very much dependent on the concentration and molecular-weight ranges, the solvent quality, the property being examined, and the type of measurement.

\section{Forced Rayleigh Scattering}

\section{E. J. Amis and C. C. Han}

So far this project has been limited to the construction of new instrumentation. In this technique a split laser beam is recombined at a small crossing angle on a sample which includes a trace of polymer labeled with a photochromic dye. This "writing" beam is pulsed to produce an interference pattern which bleaches a holographic grating in the sample. A second laser beam "reads" this pattern and is diffracted by the grating. As the labeled polymers diffuse in the sample, the grating is destroyed and thus, by measuring the intensity decay of the diffracted beam, the tracer diffusion coefficient of the labeled species can be obtained. Since the distance scale of this tracer method is very small (typically several orders of magnitude smaller than macroscopic tracer methods) it is possible to measure extremely small diffusion coefficients such as those expected for polymer molecules in concentrated solutions and melts. Recent theories of dynamics in concentrated solutions and melts should be tested by this experiment. Preliminary tests of the instrument have been very encouraging and with the installation of the detector electronics this instrument will be complete. Initial experiments will include measurement of free dye and small labeled polymers through dilute, semidilute, and concentrated solutions of various molecular weight polymers.

\section{Concentration and Polydispersity Dependence of the Static Structure Factor $s(q, c)$ and the Dynamic Characteristic Frequency, $\Omega(q, c)$ in Dilute Polymer

$$
\text { Solutions }
$$

\section{A. Z. Akcasu², B. Hammouda ${ }^{2}$, T. P. Lodge, and C. C. Han}

In all polymer characterization measurements, single chain properties such as the molecular weight and radius of gyration are obtained through extrapolation to zero concentration of measurements carried out at finite concentrations. It is clear that measurement precision can be greatly improved if single chain properties can be extracted directly from measurements carried out at finite concentrations and various polydispersities. It will be especially useful if scattering angle extrapolation can also be avoided in a scattering experiment. In this work, theoretical shape functions of static structure factor as a function of the scattering vector, $q$, and concentration, $c$, are developed and tested through light and neutron scattering data. Also, calculations of the dynamic characteristic frequency $\Omega(q, c)$ and decay rates are performed for polydispersed systems and compared to dynamic light-scattering data at the $\theta$ temperature.

\section{Single Chain Conformation in Phase Separated Diblock Copolymer Systems}

H. Hasegawa ${ }^{1}$, E. J. Amis, T. P. Lodge, and C. C. Han

Polymer properties can be greatly altered by controlling the size and shape of the phase separated domains of polymer blends or copolymer systems. It is obvious that the single chain statistics in

\footnotetext{
IGuest Worker, Kyoto University

2University of Michigan
} 
these phese seperated domeins pley en importent role in the understending of the equilibrium and nonequilibrium properties of these systems. In colleboretion with Dr. H. Hesegawn ond the Kyoto University group, we heve prepared well controlled model systems of polystyrene-polytsoprene which heve regular lamella domains. Dimensions of deuterfum lebeled polystyrone block of this diblock copolymer end elso dispersed homopolystyrene molecules were meesured et different directions reletive to the domein orientetions by smell-engle neutron scettering (SANS).

\title{
Smell Angle Neutron Scettering From Block Copolymers of h-styrene end d-styrene
}

\section{T. P. Lodge, Y. Matsushites, E. J. Amis, end C. C. Hen}

In our effort to investigate the temperature and molecular weight dependence of polymer dimensions, varfous experiments end theoretical models have been investigeted. It is true thet the chain end effects ere normelly neglected in both mean fleld end modern saling theorfes. Although perturbation theory attempts to differentiats the excluded volume effect as e function along the chain contour, it is not expected to work well in the good solvent 1 imit. In this work, we performed SANS measurements of labeled chain segments located at various positions elong the chein contour. As far as we know, this is the first time the importance (or unimportance) of neglecting the chain end effect has been tested experimentally through direct measurements of a set of well prepared and well characterized polymer samples.

\section{Static Light Scattering}

\author{
C. C. Han, H. L. Wagner, and D. B. Minor
}

The light scattering photometer has been redesigned and fabrication has been completed. Preliminary measurements show that the problem of thermal insteblifty has been overcome because of a new highly stable rotary table and isolation of the high temperature bath from the optical train. Other features which have been incorporated are a stepping motor drive for the rotary table, and a 4 mw He-Ne laser rather than the $2 \mathrm{MW}$ one used previously.

\section{Dynamics of Polymer Chains}

\section{P. H. Verdier and D. E. Kranbueh14}

The dynamic behavior of polymer chains is important to improving both cheracterization and processiblifty of polymers. The principal methods used to estimate molecular weight, and in particular gel permeation chromatography, the only method avallable at present for estimating distribution in molecular weight, are dynamical, nonequilibrium experiments which depend in part upon the relaxation of polymer chains in dilute solution. In its present state, the theory of these systeme does not allow the calculation of molecular weight directly from measured quantities; a series of calibrants of known molecular weight is required. Improvements in the theory could reduce or even remove the need for calfbrants, allowing the determination of molecular weight distribution of polymers for which callbrants are not avallable.

The processability and the pinal characteristics of both plastics and elastomers depend in large part upon the relaxation behavior of the high-polymer chains of which these materials are primarily composed. More realistic theoretical treatments of polymer melts and elastomers, which must start with more realistic treatment of the dynamical behavior of the individual chains, will lead to more efficient processing and better fabricated end products. A major shortcoming of the present theory is its inability to treat chain entanglements in a realistic way. Our present work is aimed primarily at the study of entanglement effects, using a combination of analytical and computer simulation techniques. The simulation results provide data on simple model systems and serve to guide the development of better theoretical treatments.

Work in this area is proceeding along two main lines. First, there is at present an unresolved disagreement in the literature regarding the interpretation of the time-correlation functions obtained for flexible chain molecules in the quasielastic light scattering experiment. It is not clear whether these functions exhibit the entire spectrum of chain relaxation times or are dominated by the longest relaxation time. By a combination of analytical and simulation techniques, we have shown that very slight changes in the local chain-movement rules can change the behavior of the resulting timecorrelation functions from one extreme to the other, suggesting that the existing disagreement is due at least in part to the use of insufficiently detalled chain models. These results will be published during the coming year. Second, as a step in the direction of investigation melt properties, we are carrying out simulation studies of systems of many interacting chains, with chain densities corresponding to concentrated solutions and melts. Interpretation of the results of these studies is underway, and will continue in the coming year. 


\section{F. L. McCrackin}

The values of the hydrodynamic radii of chains at infinite dilution on a cubic lattice were computed. The chains were created by a Monte Carlo simulation in which both volume exclusion and energetics of nearest neighbor interactions were taken into account. Other quantities, such as radii of gyration, were also computed for the chains. The ratio $\rho$, the radius of gyration to the hydrodynamic radius of the chains at the theta point, were compared to experimental values obtained from quasielastic light scattering and elastic light and neutron scattering. Reasonable agreement was obtained; in fact, the experimental values of $\rho$ showed better agreement with the Monte Carlo calculation than with the analytical Gaussian coil model.

The calculations were also performed for various values of the energy parameter, corresponding to good solvents. The computed values of the hydrodynamic radius are found to be in reasonable agreement with published data of the variation hydrodynamic radius of polystyrene with temperature. The results are also in agreement with calculations of A. Z. Akcasu based on the blob model of polymer solutions.

The calculations are being performed for two interacting chains. The results will be interpreted as the properties of polymers in dilute solutions. The variation of radii of gyration and hydrodynamic radii with concentration and the second virial coefficient will be calculated and compared with experiment.

\section{Span of an Adsorbed Random Flight Chain Calculated on the}

\section{Basis of the deGennes Model}

\section{R. J. Rubin}

P. deGennes showed that the one-dimensional step density distribution of monomers in his model (normalized to $N$, the number of monomers) is

$$
n(x)=2 k N e^{-2 k x} \text {, for } k>0 \text {, }
$$

where $x$ is the distance from the solution surface, and the boundary condition of the random flight propagator at the solution surface is

$$
1 / G \partial G /\left.\partial x\right|_{x=0}=-k \text {. }
$$

G. Weiss and I have derived the probability density of the span of an adsorbed polymer chain in deGenne's model. The average value of the span is

$$
\langle x\rangle=(2 k)^{-1} \ln N \text { for } N>>1 \text {. }
$$

This quantity is identically equal to the distance from the adsorbing solution surface at which the exponential step density given in Eq. (3) is equal to unity, i.e., if the equation

$$
2 k N e^{-2 k x},=1
$$

is solved for $x_{1}$, then $x_{1}=\langle x\rangle$. A paper outlining this calculation of the probability density of the span has been prepared and submitted for review.

\section{Validation of the "Weak Adsorption" Diffusion Equation Plus Boundary Condition Model of deGennes}

\section{R. J. Rubin}

We have solved the discrete lattice random walk model for a polymer chain near a surface when there is an absorbing layer at $K=-1$ and each segment of a chain configuration in 1 ayers $K=0$ to $\ell$ results in a Boltzmann weighting factor $e^{\varepsilon / k T}$ multiplying the probability of that configuration. [ $\varepsilon$ is the energy gained when any chain segment occupies any one of these $\ell+1$ layers. Thus, the weighting factor of a chain configuration in which $m$ segments 1 ie in layers $k=0$ to $\ell$ is $e^{m \varepsilon / k T}$.] We have solved the analogous diffusion equation model for this problem [this diffusion equation was first proposed by J. Pouchly', Coll. Czech. Chem. Comm. 28: 1804; 1963]. The deGennes model may be regarded as a limit of the Pouchly' model which in turn is the continuous analog of the discrete model described above. A paper comparing polymer chain behavior in these models is in preparation. 


\section{C. Sanchez}

A new statistical mechanical model for a chain liquid has been developed which shows solid-liguid transition as well as a liquid-vapor transition. This new model is a generalization of the lattice fluid model developed by Sanchez and Lacombe. The basic idea is that the mers of a r-mer can exist in efther of two states: ordered or disordered. In order for an r-mer to crystallize it must be in a particular orientation. For example, in an alkane the monomers must all be in a trans configuration to crystallize. Or if the mers have a permanent dipole, they would all have to be orfented correctly to crystallize.

We are exploring several different applications for this model. First it may prove to be an excellent model for describing glassy behavior in polymers. It should also be a good model for describing polar polymer mixtures that strongly interact. Finally, it may prove to be a relatively simple model that will be able to semiquantitatively describe the PVT properties of water.

\section{ASTM Activities}

\section{J. D. Barnes}

J. D. Barnes serves as Chairman of ASTM Subcommittee F-2.93 and 0-20.13. These subcommittees provide assistance in matters relating to the design of experiments for evaluating the precision of test methods for the parent ASTM Committees. This assistance takes the form of approval of round-robin protocols, critique of precision statements, and statistical education for task group leaders. Recent round-robin activities have included a test method for Bond Strength within Committee F-2 and a study of the density gradient technique (ASTM D-1505) for Comittee D-20. Dr. Barnes has served as a consultant to task groups working on Rockwell hardness of plastics, tensfle properties of film and sheeting, and thickness measurements using a magnetic sensor.

Dr. Barnes has recently been appointed Chairman of Subcommittee D-20.70's section on gas transmission measurements.

Or. Barnes organized a symposium on fourfer transform infrared (FTIR) methods in plastics characterization and processing that attracted five invited papers that were heard by about 75 participants at an ASTM meeting in Williamsburg, VA.

\section{Rubber and Rubber Compounding Standard Reference Materials}

\section{G. B. McKenna and G. W. Bullman}

NBS provides rubber and rubber compounding Standard Reference Materials that are used by the rubber industry for quality control in production of rubber and its processing into articles. During the past year recertification of SRMs 371 (Sulfur) and 372 (Stearic Acid) have been completed. These compounding materials are referenced in ASTM standards and used to ensure the processibility of rubber materials. The Mooney viscosity standard, SRM 388, butyl rubber, has also been renewed during the past year. This standard is widely used by the rubber industry for calibration of Mooney Viscometers which are increasingly used for on-line quality control during the production of synthetic rubbers. 
The growth in the use of polymeric materials derives from substitution for other materials as well as new applications due to the unique properties of these materials. In many applications long-term mechanical durability is critically important. The realization of increased utilization is often limited by the lack of data and models upon which to design for long-term use. Thus, the lack of adequate test methods and data for evaluation of long-term mechanical performance not only restricts applications, but also leads to over design which results in more expensive items and limits weight savings. Evaluation and control of material parameters that affect long-term performance should lead to more durable materials and, hence, reliable performance for the intended service life.

The objective of this task is to provide data, test methods, and standards for evaluation of long-term mechanical durability of polymeric materials. The approach is to identify and analyze principal failure mechanisms of commercially important thermoplastics under mechanical stress in the use environment, to determine the effects of chemical structure and fine structure morphology on mechanical properties and failure processes, and to develop constitutive relationships and models for mechanical properties to serve as the basis for accelerated test methodology and lifetime prediction.

The approach to development and verification of predictive models of mechanical performance are: (1) establish dependence of the principal failure modes of inhomogeneous deformation and crack initiation and growth on static and dynamic mechanical stress; (2) identify the principal molecular processes associated with deformation and failure and relate failure to molecular structure and fine texture; (3) evaluate the kinetic theory of bond rupture as a model for degradation of mechanical properties and for extrapolation of short-time measurements to predict long-time performance; (4) on the basis of (1), model failure by neck formation and propagation using time-dependent constitutive relations that predict the onset of inhomogeneous deformation from baseline mechanical properties characterization; and (5) expand fracture mechanics approach to viscoelastic materials to model craze and crack growth phenomena.

The National Bureau of Standards has established joint programs on mechanical durability with other Federal agencies which are fostering the application of polymeric materials in areas of national concern. For example, a joint program with the Defense Advanced Research Projects Agency is concerned with development of molecular characterization techniques for mechanical degradation as the basis for nondestructive evaluation methods. Joint projects are also executed with industries. An example is the project with Firestone Tire and Rubber Company on the effects of processing on PET structure and properties.

\section{Molecular Processes Associated with Mechanical Deformation and Failure}

B. M. Fanconi, D. L. VanderHart, S. J. Kryder, E. E. Toth, K. L. DeVries ${ }^{1}$, G. A. Bohme ${ }^{2}$ and V. D. Moche ${ }^{2}$

The elucidation of molecular processes and structure changes accompanying mechanical deformation and failure not only provides the basis for development of test methods for long-term performance and quality control, but also provides insight into the selection and design of materials with improved mechanical performance and durability. The semicrystalline structures found in some commonly used polymeric materials present significant challenges to characterization of molecular structure. Newly developed spectroscopic techniques involving ${ }^{13} \mathrm{C}$ nuclear magnetic resonance (NMR) and FTIR spectroscopies are used to elucidate molecular conformations in disordered material. Reorganization of polymer molecules during deformation affects mechanical performance. Identification of the molecular processes involved and the physical structures which result are the aim of the studies.

FTIR is used to determine relationships between the number of chain scissions produced by mechanical stress, the useful service life, and molecular weight and its distribution.

\section{Chain Scission and Mechanical Durability of Polymers}

Chain scission is an underlying molecular process associated with mechanical deformation and degradation. Previous work has established that infrared spectroscopy can be used to determine the number of carbon-carbon bonds that are ruptured under mechanical stress to form new chemical end groups.

We had applied this method to determine the number of chain scissions at fracture per measured free radical, the precursor to new end group formation. Our measurements, utilizing the improved signal-to-noise capability of Fourier transform infrared spectroscopy, established that the number of chain scissions per free radical was 1 to 2 orders of magnitude lower than previous estimates. 
Spectral studies of chein scission hove now been extended to ultre high molecular weight polyethylene damaged under fatigue loading, and to polystyrene demeged by frecturing at cryogenic temperetures. The latter study wes carried out es a function of moleculer welght and correlations with the lectron spin resonance determinations of secondary free radicel concentrations have been mede.

Specimens of uitre high moleculer weight polyethylene (UHMWPE) were fetigued under uniexiel deformation t verlous frequencies and peak stresses. The epplied loed veried sinusoldelly in time. The fetigued specimens were exemined using FTIR after follure occurred. In table 1 is shown the concentrations of carbonyl and groups found in specimens of UHMWPE fotigued ot $0.002 \mathrm{~Hz}$ and in which the peak stress varled from 30 to $45 \mathrm{MPa}$. The cerbonyl and methyl groups were the only ones thet

Table 1

\begin{tabular}{ll}
\hline Peak Stress (MPa) & $\begin{array}{l}\text { Concentration of } C=0 \\
\text { (number per gram) }\end{array}$ \\
\hline 30 & $1.95 \times 10^{10}$ \\
35 & $1.82 \times 10^{10}$ \\
40 & $2.03 \times 10^{10}$ \\
45 & $1.97 \times 10^{10}$ \\
\hline
\end{tabular}

showed an increased concentration as a result of mechanical damage. The vinyl bonds did not show eny measurable change, even though the inftial concentrations of these groups were extremely low which would facllitate the observation of small changes in concentration. This finding substantiates our earlier conclusions that the previously reported changes in vinyl group concentrations resulted from poor sampling procedures and the inltially high concentrations of vinyl groups. We also observed Increases in mothyl group concentrations but do not report these because of the large uncertelnties that result from the strong IR absorption due to methylene wagging modes in the same frequency range.

The significant result from table 1 is that the concentretion of new end groups at fallure is independent of the number of cycles, peak tress level, or time-to-fallure. By varying peak stress the timo-to-fallure and number of cycles to fallure varled by two orders of magnitude. Our findings suggest that fallure is assoclated with a critical concentration of bond ruptures and that IR measurements may serve as the basis for nondestructive test mothods. Current work is almed at substantiating the findings for different test frequencles and molecular weights.

Chain scission in a glassy polymer was examined using polystyrenes in the molecular weight range of 200,000 to $800,000 \mathrm{~g} / \mathrm{mol}$. Specimens were fractured by grinding at cryogentc temperatures and secondary free radical concentrations measured by electron spin resonance prior to warming the specimens to room temperature. The samples for infrared investigations were prepared by melt or solvent casting fllms of the fractured and reference materlal. Spectra were compared to those of the reference polymer and the increased absorption due to new end groups was converted to concentrations using literature values of the extinction coefficient. The viscosity-averaged molecular weight was also determined, and from assumptions of random chain scission the molecular welght changes were converted to number of chain scissions. In table 2 we compare the number of free radicals, the number of chain scissfons by viscosity measurements, and the IR-determined number of bond scisslons. For this glassy polymer the change in molecular weight and the IR measurements yield similar values of the number of chaln sclssions. The electron spin resonance (ESR) values are lower by a factor of 2 to 3 indicating that a secondary free radical may produce more than one chain scission. The insensitivity of the number of chain scissions to molecular welght can be related to grinding at low temperature where large scale molecular motion is prohibited.

Table 2

\begin{tabular}{llcc}
\hline$M W \times 10^{3}$ & $\begin{array}{l}\text { Viscometry Ruptures } \\
\text { number per } \mathrm{cm}^{3} \times 10^{-16}\end{array}$ & $\begin{array}{c}\text { ESR R / } \mathrm{cm}^{3} \\
\times 10^{-16}\end{array}$ & $\begin{array}{c}\text { IR Chain Sc1ssions } \\
\text { number/cm } \times 10^{-16}\end{array}$ \\
\hline 220 & $17.0 \pm 3$ & $4.5 \pm 2$ & $17 \pm 3$ \\
637 & $11.0 \pm 3$ & $6.3 \pm 2$ & $22 \pm 4$ \\
916 & $15.0 \pm 4$ & $5.0 \pm 2$ & $24 \pm 4$ \\
\hline
\end{tabular}

Examination of Drawn PET Fibers by Solid State NMR

This work is a continuation of work begun in FY8I and is a foint effort with the Firestone Tire and Rubber Company. The interest is to learn how to control shrinkage of PET fibers in tires during rubber curing while maintaining mechanfcal strength. 
In previous ${ }^{13} \mathrm{C}$ NMR studies on these materials, it became clear that a better understanding of the relationship between morphology and orientation in drawn PET fibers would be very helpful. Using the ${ }^{13} \mathrm{C}$ NMR method, one could get a reasonable description of overall fiber orientation. However, temperature-dependent studies showed significant heterogeneity of molecular mobility among the oriented aromatic groups in a drawn sample. This underscored the importance of understanding the nature of the distinction between crystalline and noncrystalline phases in these materials. Several NMR techniques were applied to this question making use of magic angle sample spinning spectra, relaxation time measurements, orientational measurements, and spin diffusion measurements. Although $x$-ray techniques indicate rather small crystallite sizes, particularly along the C-axis ( 3.5 to $6 \mathrm{~nm}$ ), calculations of crystallinity from the $x$-ray data contain a great deal of uncertainty.

In FY82 a different NMR approach to the question of crystallinity was undertaken. This approach was based on the phenomenon of spin diffusion (really diffusion of spin polarization). If a well-defined spin polarization gradient can be produced in a 2-phase system such as PET, and if the system can be observed for a sufficiently long time thereafter (but a time short compared to the longitudinal relaxation time, $T_{1}$ ), the rate at which spin polarization equilibrium is attained will indicate the size of the domain(s) since the spin diffusion constant is approximately known.

Protons (and not ${ }^{13} \mathrm{C}$ ) exhibit strong spin-diffusion characteristics. Therefore, much effort was put forth finding the optimal conditions for generating magnetization gradients between the two phases of the drawn PET fibers. This problem is not easy because very few chains, below $T_{g}$ ' in PET undergo significant motion on the NMR timescale of $10^{-5} \mathrm{~s}$; therefore, it is difficult to create polarization gradients below $\mathrm{T}_{\mathbf{g}}$. Above, $\mathrm{T}_{\mathbf{g}}$, this task is easier but the sample also changes irreversibly. Although some compromises have to be made, current efforts make use of differences in rotating frame relaxation times between the two phases in order to establish a polarization gradient. Preliminary results suggest that equilibration times between the two phases are not a very sensitive function of post-annealing history.

In a quantitative way, this suggests that there is no significant reduction in surface area between the two phases. Moreover, annealing is not significantly changing the sum of the crystalline plus noncrystalline domain size in the direction of minimum thickness. The distances for this sum deduced from the spin diffusion rates are in range of 10 to $15 \mathrm{~nm}$. This is in accord with $x-r a y$ results. The picture of drawn, annealed (at constant length) fibers is that annealing increases the regular ordering in the drawn fibers but it does not result in substantial thickening of the crystalline regions. Moreover, in annealing, only those chains which were originally well-oriented in the draw direction are involved in the perfecting of order by annealing. Orientation in the noncrystalline region remains pretty much independent of annealing with a slight disordering at high annealing temperatures. This is probably the reason that modulus tends to degrade at high annealing temperatures even though crystalline orientation remains high.

It should be noted that Dr. V. Mochel from Firestone Tire and Rubber Company spent a week at NBS working on the project in FY81 and that Dr. K. W. Zilm from Utah Research Institute spent a month in FY82 working on some of the PET proton experiments.

Characterization of Ultrahigh Modulus Materials

This work is aimed at developing techniques to characterize structural features of ultrahigh modulus polymeric materials. This year a collaboration effort was initiated with Professor Ian M. Ward of the University of Leeds in England. Professor Ward has extensive sample preparation facilities for producing highly drawn materials. We are exploring the possibilities that NMR may be able to obtain information about tie molecules, those molecules that bridge one crystalline lamella to another, which are regarded as essential components for achieving a high modulus. Studies are underway investigating effects of molecular weight, draw ratio, and draw temperature. One of the measures which may relate to tie molecules is longitudinal growth of ${ }^{13} \mathrm{C}$ magnetization in the intermediate time regime. This magnetization has the appropriate orientation to correspond to tie molecules. In preliminary investigations to date, the number of chain segments corresponding to this magnetization is roughly proportional to the noncrystalline fraction in all drawn materials. A significant observation which lends credence to this pursuit is the smaller fraction of material associated with this magnetization in an unoriented sample where we would expect fewer tie molecules traversing the noncrystalline region.

\section{Investigations of Cellulosic Materials used in Paper Making}

A cooperative effort with the Bureau of Engraving and Printing is aimed at identifying more durable and less expensive substitutes for currency papers which are presently made from linen and cotton cellulosic materials. Wood pulps are a much more available source of materials but the mechanical characteristics of these papers is generally inferior. A fundamental question is how the morphology of paper affects its mechanical properties and durability. In FY81 we used magic angle spinning (MAS) ${ }^{13} \mathrm{C}$ NMR to investigate any modifications to the surface area of cotton and wood pulp materials as a result of wet beating. (Wet beating is the process whereby pulps are sufficiently plasticized in order to make paper.) One of the characteristics of the ${ }^{13} \mathrm{C}$ spectra is that the presence of water in various amounts produces only minor changes in the spectra. Therefore, in an 
effort to understand the microstructure of cellulose better, an intensive effort was put forth to obtain more information about the interaction between $\mathrm{H}_{2} \mathrm{O}$ and cellulose. A significant objective in these studies is to answer the question of whether disorder in native celluloses is associated with only disorder on the surface of cellulose fibrils, or disorder also exists in three-dimensional noncrystalline regions. The spin diffusion studies combined with $13 \mathrm{C}$-MAS experiments should shed significant light on this question. To date, the principal material for study has been cotton. The transfer of magnetization between water and cellulose, via spin diffusion, has been witnessed as a function of water content. It has been established by ${ }^{13} \mathrm{C}$ NMR that proton magnetization gradients in the cellulose can indeed exist, thereby leaving. open the possibility that three-dimensional disordered regions exist.

\section{The Distribution of Stresses in Crazes at Crack Tips}

\section{E. Passaglia}

When computing any of several criterla for crack-growth (such as critical crack opening displacement or work to fracture) in crazing polymers, it is necessary to know the distribution of stresses within the craze. From this distribution the craze displacement profile may be calculated, giving crack opening displacement directly, or work to fracture by the product of stress and displacement.

Many workers have used the Dugdale model for the craze which assumes a constant stress within the craze. On the other hand, by direct determination from the electron microscopic observation of the displacement proffle in either continuous crazes or crazes at crack tips, Kramer and co-workers [1,2] have shown that the stress is not constant within the craze. Most typically, it has a peak at the craze tip, and may also rise at the crack tip.

It becomes of interest, therefore to develop a means for determining the stress distribution in a craze. This may be done if the craze is modeled as a necking and drawing process. It has been shown that this leads to reasonable results [3]. In this model, in a moving crack, material at the craze tip is assumed to fibrillate, with the fibrils drawing out from the unfibrillated substrate, as the necked polymer is drawn from the unnecked in a drawing process. The rate of craze displacement thus corresponds to the rate of drawing, and the stress will be a function of this rate.

Consider now a crack with a craze zone at its tip propagating unfformly under the action of an applied stress intensity factor $K$. If under this condition of uniform translation the craze stress cancels the stress singularity that would otherwise be present, the displacement may easily be calculated [4]. (It is noted that this implies a critical crack opening displacement or work to fracture criterion for crack advance.) Now denoting the displacement by $w$, and the crack velocity by $v$, from the condition of unf form translation we have

$$
(\partial w / \partial t)_{X}=-v(\partial w / \partial X)_{t}
$$

where $X$ is a position coordinate. With an origin at the crack tip and coordinate $\xi$,

$$
\partial w / \partial t_{X}=v \partial w / \partial \xi
$$

From the displacement, $\partial w / \partial \xi$ is easlly evaluated. This gives

$$
\partial w / \partial \xi=\operatorname{ce} / 2 \pi \int_{0}^{\alpha} \sigma(\zeta)(\xi / \zeta)^{1 / 2} d \zeta / \xi-\zeta,
$$

where $C e$ is the plane strain compliance of the uncrazed material, $\alpha$ is the craze length, $\sigma(\zeta)$ is the stress distribution in the craze, and $\zeta$ is an integration varlable. Now, since the stress is assumed to be a function of the rate of change of displacement at the point $\xi$ we have using Eqs. (2) and ( 3 ) a form of integral equation for the stress distribution. A solution algorithm is the following:

(1) Assume a relation between stress and rate of change of displacement. From the hypothesis that crazing may be modelled as necking, this relation may be obtalned for curves of yield and drawing stress as functions of the rate of drawing.

(2) Assume a stress distribution and calculate $\partial w / \partial \xi$ from Eq. (3).

(3) From Eq. (2), and the assumed relation between stress and rate of draw, calculate $\sigma(\xi)$. This should be the same as the assumed stress distribution in (2).

Several attempts have been made at such a solution with these preliminary and tentative results. 
(1) A uniform stress distribution does not work, which is not surprising.

(2) A stress distribution, which has a peak at the craze tip, is constant over most of the craze, and drops to zero at the crack tip, gives a rough approximate solution to the problem. Work on this problem will continue.

[1] Lauterwasser, B. D. ; Kramer, E. J. Phil. Mag. A39: 469; 1979.

[2] Wang, W. V.; Kramer, E. J. J. Mater. Sci.; in press.

[3] Verheulpen-Heymans, N. Polymer 21: 97; 1980.

[4] Schapery, R. A. Int. J. Fract. 11: 141; 1975.

\section{A New Test Method for Environmental Stress-Crack Resistance}

\section{J. M. Crissman and L. J. Zapas}

We reported previously (Polymer Science and Standards Division 1981 Annual Report) that over the past several years there has been renewed interest in the development of a new test method for determining the environmental stress-crack resistance (ESCR) of ethylene based plastics. As part of our studies concerned with long time behavior, durability, and fracture of polyethylenes, we have examined a number of possible test methods which yield information concerning ESCR. One of these methods we propose as a viable alternative to either of the test methods currently recommended by ASTM for determining ESCR. The proposed new test method has advantages over existing methods. (1) The specimens are maintained in a fixed and constant geometry. (2) The testing time is reduced. (3) The experimental scatter in the data is reduced.

The proposed new test combined features of the two most widely accepted test methods for ESCR, ASTM D-1693 and ASTM D-2552. The specimen is bent around a metallic form having a fixed radius of curvature. At the same time it is subjected to a constant applied load at an elevated temperature. During the past two years work has proceeded to determine an optimum set of test conditions for polyethylenes having widely different molecular weights. Test variables being considered are the specimen thickness, bend radius, applied load, and temperature. Four different polyethylenes ranging in molecular weight from $10^{5}$ to $4 \times 10^{6}$ have been used in this work. Based upon the test results for more than 400 specimens the following set of test conditions was chosen.

$\begin{array}{ll}\text { Specimen Thickness } & 0.100 \text { to } 0.125 \mathrm{~cm} \\ \text { Bend Radius } & 0.55 \mathrm{~cm} \\ \text { Applied Stress } & 5 \mathrm{MPa} \\ \text { Temperature } & 60 \text { to } 75{ }^{\circ} \mathrm{C}\end{array}$

A description of the test method, as well as a summary of the test results, including the appropriate statistical data, was presented before Committee D-20.12.03 of ASTM at their June 1982 meeting in Toronto, Canada. Subcommittee D-20.12.03 is currently considering carrying out a round-robin test using the new test method.

Creep and Recovery of UHMWPE at Very Small Deformations: A New One-Dimensional Constitutive Equation

L. J. Zapas and J. M. Crissman

Since the early work on the mechanical properties of natural polymeric materials well over one hundred years ago [1,2] it was found that reproducible data could be obtained only after the specimens had been mechanically preconditioned. Leaderman in his classical publication on the "...Properties of Filamentous Materials..."[3] reported that even for synthetic polymers the Boltzmann superposition could be obtained only when the materials were subjected to mechanical preconditioning. The necessity of preconditioning the material raises questions as to what one measures as material properties of the virgin or unconditioned polymer. Another difficulty has been the inability of the BZK Theory [4] to describe quantitatively the two-step stress-relaxation experiments where the second step is smaller or equal to one half the value of the first step.

To better understand this behavior we have obtained data on unconditioned specimens of an ultra high molecular weight polyethylene (UHMWPE) which were subjected to a variety of different strain histories in uniaxial extension. The observed behavior led us to a one dimensional constitutive relation which describes very well all of our experimental results. In the new derivation, the assumption is that the undistorted state of the material depends upon the stress history. This means that the strain which the material itself sees is not that which the observer sees. For very small deformations, of the order of one percent and below, the constitutive relation is given by the following equation: 


$$
\varepsilon(t)=\phi\left(\int_{-\infty}^{t} g(\sigma(\xi)) d \xi\right)=J(\sigma(t), 0)+\int_{-\infty}^{t} J_{*}(\sigma(\xi), t-\xi) d \xi,
$$

where $t(t)$ is the strain the observer sees, $o(t)$ is applied true stress, $\phi($.$) is a functional, and$ $J(o, t)$ is a nonlinear compliance. $J_{*}(\ldots)$ denotes the partial derivative of $J(\ldots)$ with respect to the second argument. When $\phi($.$) is zero and J(0, t)$ is 1 inear in $\sigma$, equation (1) reduces to the Boltzmann equation. The functionals and $J$ can be obtained from a series of creep and recovery experiments.

We have carried out such a serfes of experiments on the UHMWPE and it is found that the functions $\phi$ and $\mathrm{J}$ can be represented rather well by the expressions

$$
\phi\left(\int_{0}^{t} g(\sigma(\xi)) d \xi\right)=\left(\int_{0}^{t} g(\sigma(\xi)) d \xi\right)^{1 / 3},
$$

and

$$
J(\sigma, t)=f_{1}(\sigma)+f_{2}(\sigma) t^{1 / 3} .
$$

Relations (2) and (3) along with equation (1) give interesting prediction that recovery data, obtained from a series of creep experiments done at constant stress but different values of the duration of the step, can be superposed onto master curve with predetermined shift factors along both the strain and time axes. This was done and the data points on the resultant master curve formed a very narrow band of the order of 10 percent or less, which is as good as the reproducibility of the creep curves from specimen to specimen. We have also calculated the behavior for multistep creep and recovery experiments and the agreement was within 2 percent of the experimental values.

Since Eq. (1) is nonlinear, it cannot be inverted in order to determine the stress as a function of the strain history. However, the consistency of Eq. (1) can be determined from experiments where the stress is measured as a function of the strain history, by assuming that, infinitesimaily in time, the motions are obtained from constant stress experiments. This was done for single- and two-step stress-relaxation experiments and the predicted strains were within a few percent of the actual applied strains. For a two step in strain experiment, where the second step was very close to half the strain of the first step, the BZK prediction gave results for the second step which were higher by 45 percent, while the strains calculated using Eq. (1) fell within 3 percent of the experimental values. The proposed viscoelastic equation appears to have the necessary elements in order to be capable of describing quantitatively both the creep and recovery behavior of a wide range of materials.

[1] Weber, W. Ueber die elasticitat der seidenfaden. Pogg. Ann. Physik 4: $247 ; 1835$.

[2] Weber, W. Ueber die elasticityt fester Korper. Pogg. Ann. Phys1k 24: 1; 1841.

[3] Leaderman, H. Elastic and creep properties of filamentous materials and other high polymers. The Text1le Foundation, Washington, DC; 1943.

[4] Bernstein, B. ; Kearsley, E. A. ; Zapas, L. J. Trans. Soc. Rheol. 7: 391; 1963.

A Study of the Morphology and Mechanfcal Propertles, and Their Dependence on Thermal History and Processing of Uitra High Molecular Weight Polyethylene

J. M. Crissman and F. Khoury

Ultra high molecular weight polyethylene (UHMWPE) is used in the manufacture of a variety of orthopedic joint prostheses. One factor which $11 \mathrm{mits}$ the lifetime of a prosthesis is the durability of the UHMWPE component. The length of time between device Implantation and fallure depends upon t1me dependent processes which result from the clinical loading environment. In clinical use, fallure of the polymeric component has been reported to occur as a result of creep, wear, fatigue associated with wear, and even fracture. Both the mechanical properties and durability are determined by the polymer chemistry and morphology related features. Factors such as crystallinity, orientation, spherulite size and texture, as well as molecular weight and molecular weight distribtion all influence mechanical performance. Under contract with the Bureau of Medical Devices, Food and Drug Administration, we are in the third year of a study concerned with the relationship between the morphology of UHMWPE and the mechanical behavior. An important aspect of this work is the determination of the mechanical behavior variability which can be brought about by changes in processing conditions.

A summary of the earlier work done in this project is contained in the Polymer Science and Staridards Division 1981 Annual Report. The initial stages of the work dealt mainly with (1) characterization of the raw polymer powder, (2) establishment of procedures for compression molding, 
under vacuum, sheets and cylinders of the UHMWPE, (3) characterization of the processed polymer, and (4) initiation of preliminary experiments on the creep, stress-relaxation, and fatigue behavior of UHMWPE. We have previously reported on the morphology, density, and uniaxial stretching and recovery of compression molded sheets made from a UHMWPE polymer (UH-1) having an intrinsic viscosity of $\sim 25$ $\mathrm{d} 1 / \mathrm{g}\left(\mathrm{MW} \sim 4 \times 10^{6}\right)$. The sheets were molded at $200{ }^{\circ} \mathrm{C}$ and then cooled to room temperature at $\sim 1{ }^{\circ} \mathrm{C} / \mathrm{min}$. The density of these sheets was in the range 0.934 to $0.936 \mathrm{~g} / \mathrm{cm}^{3}$, the higher density corresponding to a weight percent crystallinity, $x$, of 59.7 percent. Sheets which were quenched from the melt into cold water have a density of $0.923 \mathrm{~g} / \mathrm{cm}^{3}(x=51.5$ percent). In order to determine whether appreciable higher crystallinities can be achieved with this polymer, sheets of the slowly cooled material were annealed in nitrogen at successively higher temperatures in the range 125 to $135{ }^{\circ} \mathrm{C}$. The highest density attained was $0.942 \mathrm{~g} / \mathrm{cm}^{3}(x=63.4$ percent $)$.

Since molecular weight can also be an important factor in determining mechanical behavior, in FY82 the work was expanded to include a lower molecular weight UHMWPE (UH-2) having an intrinsic viscosity of $\sim 18 \mathrm{dl} / \mathrm{g}$ (MW $\left.\sim 2 \times 10^{6}\right)$. Sheets of the UH-2, prepared under the same three sets of conditions described for the UH-1, had densities which ranged from $0.925 \mathrm{~g} / \mathrm{cm}^{3}(x=52.2$ percent) for the quenched polymer to $0.953(x=70.7$ percent) for the annealed polymer. Clearly, the crystallinities of the molded sheets of the lower molecular weight polymer are not greatly different than those of the UH-1 polymer.

The densities (crystallinities) of the compression molded sheets of both polymers are comparable to those of commercial prostheses. The densities of specimens cut from various regions of five acetabular cups and five tibial plateaux were determined, and were found to fall in the range 0.923 to $0.953 \mathrm{~g} / \mathrm{cm}^{3}$, which spans the entire range found for the compression molded UH-1 and UH-2. It should be borne in mind, however, that the prostheses were subjected to sterilization by irradiation which may have effected their densities.

In addition to measuring the density of the sheets of UH-2 molded at $200{ }^{\circ} \mathrm{C}$ and slowly cooled to room temperature, we observed the following similarities with the sheets of UH-1. First, a memory of the granular texture of the raw polymer is retained in the molded specimens. Second, specimens stretched uniaxially at 23 and $37{ }^{\circ} \mathrm{C}$ did not exhibit necking, and showed considerable recovery when released at strains below the breaking strain. For example, specimens stretched at a strain of $\sim 0.2 / \mathrm{min}$ to strains of 2 and 3 , and which were then released, had, after 24 hours storage at room temperature, residual strains of 1.09 and 1.86 , respectively. Wide-angle $x$-ray diffraction patterns of such specimens revealed the presence of reflections associated with the monoclinic polymorphic form of polyethylene in addition to the reflections associated with the predominant orthorhombic form. The particular aspect of the deformation of sheets of UH-1 and UH-2 which is currently being investigated is the apparent preferential deformation of the specimens (during the early stages of drawing) at boundaries which delineate the "interfaces" between the "sintered grains" of raw polymer.

In the area of mechanical properties studies, work centered on the characterization of the creep and stress-relaxation behavior of both the UH-1 and UH-2 polymers as a function of thermal treatment. Particular emphasis was placed on single and multi-step creep recovery experiments at very small deformations in uniaxial extension. It was found that, even at levels of strain of 0.001 and below, the stress-strain behavior is nonlinear and the recovery behavior cannot be described by the Boltzmann Superposition Principle. To better understand the behavior of the UHMWPE a series of creep recovery experiments was done on unconditioned) specimens in which either the applied stress was maintained constant and the duration of the creep step was varied, or the duration of the creep step was. held constant and the applied stress was varied. The observed behavior led us to a new one dimensional constitutive relation which describes very well all our experimental results and the details of which are described elsewhere in this Annual Report.

Work was also continued to characterize the environmental stress-crack resistance of the two UHMWPE polymers. It was found that in general the slowly cooled UH-1 and UH-2 had a longer lifetime in the surface active agent that did either the quenched or annealed UH-1 and UH-2. Moreover, the ESCR of the UH- 2 was better than that of the UH-1 under the same set of test conditions.

The Effects of Mechanical Deformation on Glassy Polymers

\section{G. B. McKenna and A. J. Kovacs ${ }^{3}$}

According to Struik [1], large deformations of aged polymer glasses can result in erasure of the prior aging. From his experimental results, Struik concluded that large stresses (or deformations) enhance the molecular mobility of the aged glass to the point that the mechanical properties are similar to those of a freshly quenched glass. He hypothesized that the deaging of a glass should be accompanied by changes in (free) volume of a magnitude appropriate to the enhanced mobility. We refer to these as structural effects, and to date there has been no experimental confirmation of them. 
Deformation of compressible solids by tension and compression results in relatively large changes in volume (first order effects) [2]. On the other hand, torsional deformations are predicted irom finite lasticity theories to result in much smaller volume changes (second order effects) [3]. We refer to these as mechanical effects. Because the volume changes expected in torsion are much smaller than those accompanying tension or compression, this geometry is more sultable to studying the effects of deformation on the structure of polymer glasses.

There are two major objectives for this project: the measurement of the effect of torsion on (a) the volume of a glassy sample and (b) on its molecular mobility. As for the second theme, it is of particular importance to determine if any variation of the molecular mobility is accompanied by a dilation when deaging (destabilization) is provoked by a deformation greater than a critical value. In the case of a positive response to this question, one must then determine the magnitude of the dilation in subtracting out any mechanically induced volume changes due to the torsion. If the response is negative, we will attempt to determine the possible origin of the destabilization and the parameters which control it.

In order to accomplish the objectives, we have spent the past year in bullding and perfecting a torsional dilatometer (TD). With the help of this instrument we will be able to determine the effects of torsion on the volume of polymer glasses (a) in the absence of any destabilization (mechanical effect) and (b) when the deformation is accompanied by a simultaneous increase in molecular mobility (structural effect).

The apparatus has been conceived to be able to measure simultaneously, the torque, the normal force and the variation of the volume of a sample subjected to torsional deformations controlled with preciston, while the length of the sample is held constant. The dilatometer is filled under vacuum with mercury through a capillary having a calibrated cross section. This is illustrated schematically in figure 1.

An electric servo-motor is used to turn the sample (fig. 2). The motor is commanded by a digital servo-system represented schematically in figure 3. The system permits the performance of single- and multiple-step stress relaxation experiments at strains varying from $\gamma=0.001$ to $0.25 \pm 8 \times 10^{-5}$. The maximum time required to introduce the step is less than 1 second. Constant rate of deformation experiments can also be carried out.

The temperature of the dilatometer is maintained constant by circulation from a water bath maintained to $\pm 1 / 500{ }^{\circ} \mathrm{C}$. This precision is necessary to give a sensitivity of the dilatometer to relative volume changes of the order of $\pm 10^{-6}$. The accuracy on the torque is \pm 3.5 percent.

[1] Struik, L. C. E. Physical aging in amorphous polymers and other materials. Elsevier Publishers, Amsterdam; 1978.

[2] Timoshenko, S. P.; Goodier, J. N. Theory of elasticity. 3rd ed. McGraw-H111: New York; 1970.

[3] Murnaghan, F. D. Finite deformation of an elastic solid. J. Wiley \& Sons: New York; 1951.

\section{Mechanical Relaxation of Liner Materials in Acetic Acid}

\section{J. C. Phillips}

Polymers of varied chemical constitution and design are increasingly used as barrier materials in hazardous materials storage ponds and landfills. The essential purpose of these materials is to prevent or impede the leakage of liquid waste products into the underlying soil and ultimately polluting the groundwater. In order to evaluate the efficacy of liner materials one has to better characterize the transport and mechanical properties behavior of these polymeric materials. The initial investigation addressed the former problem using low and high density polyethylene and the latter problem has been studied using stress relaxation measurements to test the strain performance of three liner materials (high density polyethylene, HDPE; chlorinated polyethylene, CPE; polyvinyl chloride, PVC) both in air and acetic acid.

The experimental data for stress relaxation were obtained at temperatures of 30,50, and $72{ }^{\circ} \mathrm{C}$. At the latter temperature $\left(72^{\circ} \mathrm{C}\right.$ ) stress relaxation experiments were performed on HDPE as a function of elongation and concentration of acetic acid/ $\mathrm{H}_{2} \mathrm{O}$ mixtures. Environmental stress cracking (ESC) experiments at 60 and $90{ }^{\circ} \mathrm{C}$ were done for HDPE using acetic acid and Igelpal as ESC agents. Additional experiments of recovery were also performed for the three linear materials. In air at 30 , 50 , and $72^{\circ} \mathrm{C}$, HDPE, PVC, and CPE exhibited a normal relaxation behavior over the experimental time and strain range, i.e.,

$$
\log E(t)=\log K+\alpha \log t
$$




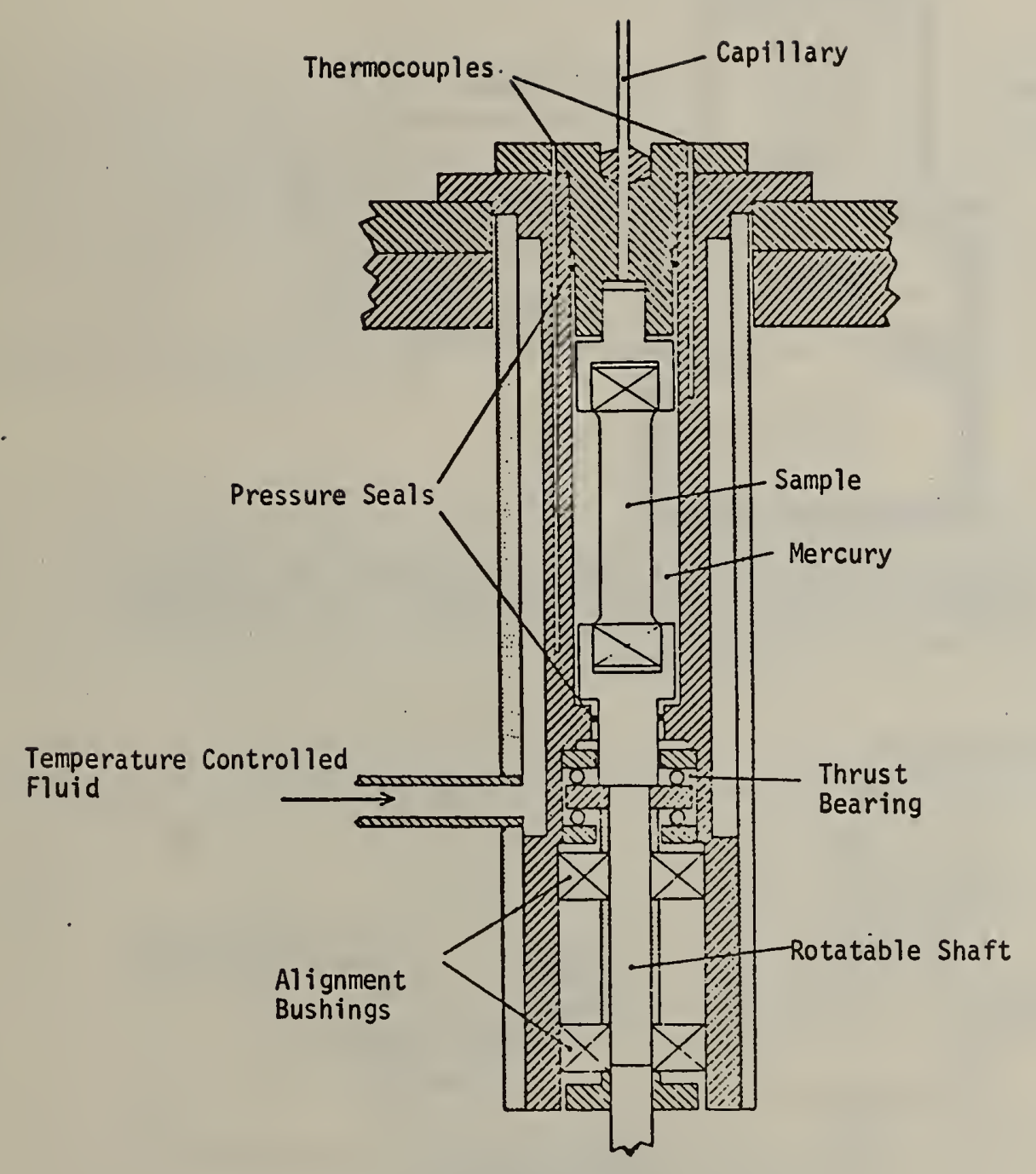

Figure 1. Schematic of the Torsional Dilatometer. 


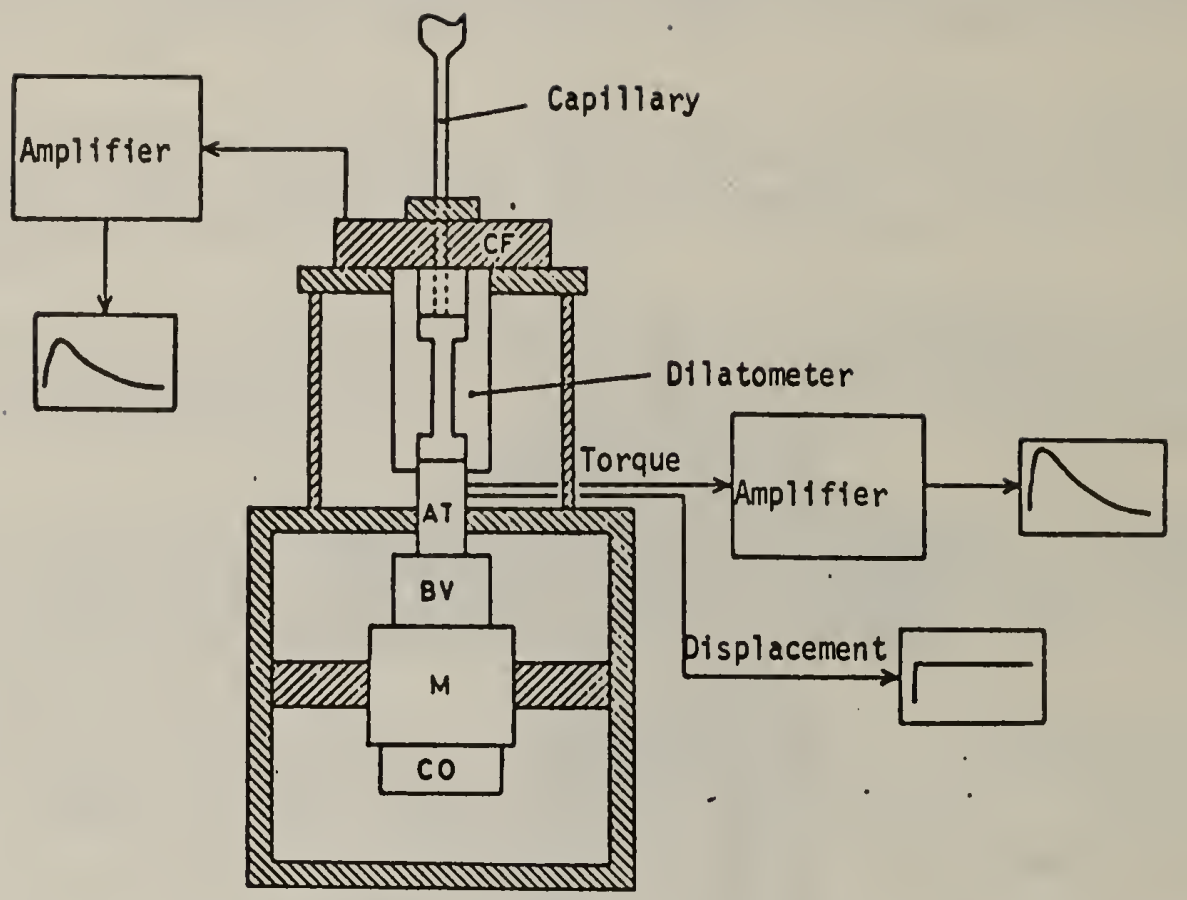

Figure 2 General schematic of the torsional dilatometer. $C O=$ optical encoder,$M=$ motor; $B V=$ high precision gears; $A T=$ drive shaft; $\mathrm{CF}=$ normal force transducer.

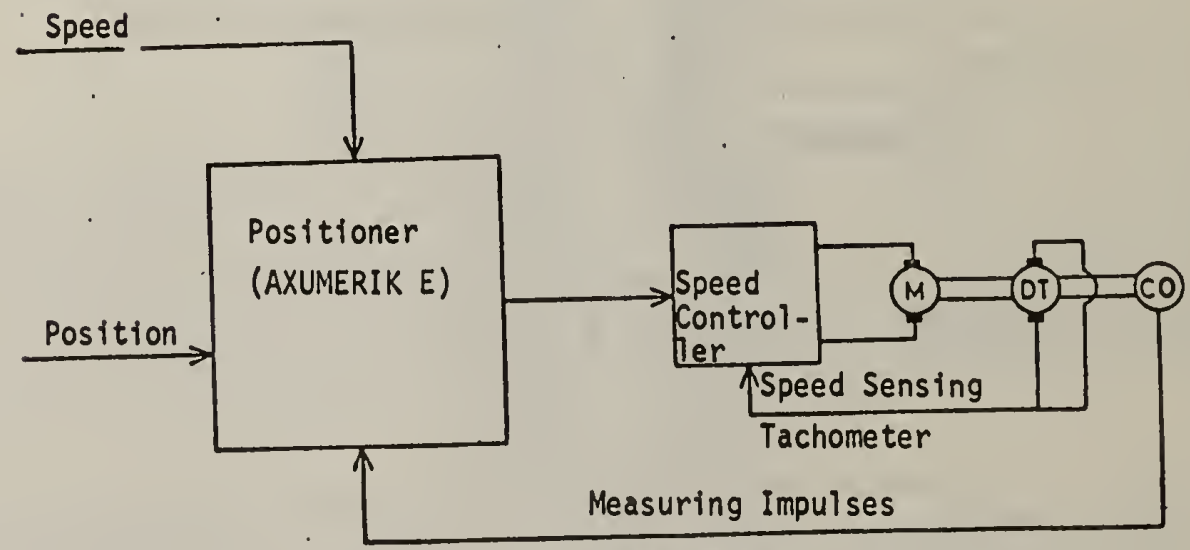

(Speed \& Position)

Figure 3. Schematic of servo-control system for torsional dilatomer. $M=$ motor; DT = tachometer; $\mathrm{CO}=$ optical encoder. The Axumerik E represents the digital system which performs the calculations used to control the angular position. 
where $K$ is a function of strain $\varepsilon$ and $\alpha$ is the slope of the log-log plot. For HDPE the slope shows little if any variation with temperature while the slope for PVC is slightly more negative and CPE has an even greater negative slope. In acetic acid the modulus was represented as a function of the characteristic time, $t-t_{0}$, of the sorption process ( $t_{0}=$ the time for initialiy bringing the liquid in contact with the sample), i.e.,

$$
\log E(t)=\log K^{\prime}+\beta \log \left(t-t_{0}\right)
$$

At 30,50 , and $72{ }^{\circ} \mathrm{C}, \beta$ approximately equals $\alpha$ for HPDE. At $72{ }^{\circ} \mathrm{C}$, the modulus for CPE was not measurable due to an extremely rapid drop in modulus to zero immediately after stretching the sample. The only significant changes of modulus in acetic acid for PVC versus that in air also occurred at $72{ }^{\circ} \mathrm{C}$ for a strain of $\varepsilon=0.89$.

This investigation attempted to show how the relaxation modulus may be used as a distinguishing parameter for different liner materials under the influence of a given stress relaxation modulus, $i . e .$, the ratio of the modulus in liquid to that in air versus time. Further refined studies may show this parameter to be quite useful in characterizing the mechanical relaxation of potential 1 iner materials.

\section{Crystal Growth Kinetics and the Lateral Growth Habits of Polyethylene Crystals}

\section{E. Passaglia and F. Khoury}

It is well known that when polyethylene crystals are grown from solution they become more elongated in the direction of the b crystallographic axis the higher the temperature of growth. At high temperatures the crystals are bounded laterally by (110) and (200) crystallographic faces. The latter faces become longer relative to the (110) faces the higher the crystalization temperature. At low temperatures and in some solvents, notably xylene, the (200) faces are not present. The crystals are bounded only by (110) faces, and they exhibit the familiar lozenge habit. The ratio of the length of the crystal in the b crystallographic direction to its length in the a crystallographic direction, which we denote by $r$, the aspect ratio, is thus a measure of the habit of the crystal. The value of $r$ ranges from about $0 . \overline{6} \overline{6}$ for the lozenge habit to five or higher at high temperatures in poor solvents.

At steady state growth, $r$ is related to the rates of growth normal to the (110) and (200) faces of the crystal by the simple equation

$$
\underline{r}=G^{110} /\left(\operatorname{Cos} \theta G^{200}\right)
$$

where $\mathrm{G}^{110}$ and $\mathrm{G}^{200}$ are the rates of growth normal to the two faces, and $\theta$ is the angle between the b direction of the crystal and the normal to the (110) face. Since $r$ varies with temperature, $G^{110}$ and G200 must have different temperature dependencies. The growth rate $G$ depends upon several material parameters, the most important of which are the surface energies, and several theoretical parameters, most important among which are the length of the substrate for nucleation, $L$, in regime I crystallization and $\phi$, the fractional assignment of the free energy of crystallization to forward and backward reactions in the stem deposition process in both regimes I and II.

An analysis of the temperature dependence of crystal habits on the basis of Eq. (1) allows the possibility of investigating the values of the various parameters--particularly the determination of the relative values of surface energies associated with stem deposition and folding for the two crystal surfaces, and the effect of $\phi$ upon the analysis.

Writing Eq. (1) explicitly we obtain for regime I crystallization $\left(r_{I}\right)$ and regime II crystallization $\left(r_{I I}\right)$ :

$$
\begin{gathered}
\ln r_{I}=\ln C_{I}+\ln \frac{L_{110}}{L_{200}}+\frac{2(a / 2) b \phi \sigma_{e}^{110}}{k T}(1-\rho) \\
+\frac{4 d 110 \sigma_{e}^{110_{\sigma}^{110}}}{\Delta h \Delta T k T / T_{d}^{0}} \frac{a / 2}{d_{110}} \gamma \rho-1 \\
\ln r_{I I}=\ln C_{I I}+\frac{(2 \phi-1)(a / 2) b \sigma_{e}^{110}}{k T}+\frac{2 d_{100} \sigma_{e}^{110} \sigma^{110}}{\Delta h \Delta T k T / T_{d}^{0}} \frac{a / 2}{d} \gamma \rho-1 ; 110
\end{gathered}
$$


where the symbols have the following meaning:

$C_{I}, C_{I I}: \quad$ ratios of lattice parameters (slightly temperature dependent).

$L_{100}, L_{200}$ : The length in which one nucleation event occurs for the (110) and (200) faces in regime I crystallization.

$a, b, d_{110}: \quad$ The lengths of the and $b$ axes, and the (110) interplanar spacing in the crystal.

$\sigma^{110}, \sigma_{e}^{100}$ : The lateral and end surface free energies associated with the deposition of a stem and folding of the chain on the (110) surface of the crystal.

$\rho: \quad \sigma_{e}^{200 / \sigma} e^{110}$

Y: $\quad \sigma^{200 / \sigma^{100}}$

$\Delta h:$

Heat of fusion of extended chain crystal, per unft volume.

$T_{d}^{0}:$

The dissolution temperature of the extended chaln crystals for the given molecular weight and solvent system.

$\phi:$

Parameter that assigns a fraction of the free energy of crystallization to the forward reaction and the remainder $(1-\phi)$ to the backward reaction in each deposition step.

Before analyzing experimental data according to Eqs. (2a) and (2b) some decision must be made about $L_{110}$ and $L_{200}$. First, if these correspond to the lengths of the respective crystal edge, then it is easy to show that growth rates are exponential, and not linear as observed experimentally. In order to obtain linear growth, $L$ has to be a constant. Second, whatever the physical significance of $L$, we have no basis for assigning a different value to $L_{110}$ and $L_{200}$. Hence we take them to be equal.

The analysis was carried out on data by Blundell and Keller [1] and Valenti and Pedemonte [2] for the crystallization of Marlex 6009 in xylene at various temperatures and in 0.01 percent and 0.1 percent solutions. This is the most extensive data set available for which $T_{d}^{0}$ is known as a function of concentration and molecular weight.

The results are as follows:

(1) If $\gamma=1,1.0 .$, If the lateral surface energy for deposition on (200) is the same as that for deposition on (110), and if $p$ is not temperature dependent, no fit can be obtained for any values of $p$. This holds for both regimes and for all values of $\phi$. This means that at least two parametars for any given $\phi$ are necessary to fit the data. Two parameters can be obtained in the following two ways: (a) $\gamma=1, \rho$ is temperature dependent, $\rho=\rho_{0}(1+$ CT); (b) $\gamma \neq 1 ; \rho \neq 1 ;$ but both are temperature independent.

(2) Data analysis for case (a).

Analysis of the data in this case gives the following results: (a) The value of $\rho_{0}$ depends upon the value of $\phi$ chosen for the analysis, and the crystallization regime assumed to control growth. However, in all cases, at a reference temperature of $338 \mathrm{~K}, \rho_{0}>1$ its value ranging from 1.01 to 1.1 depending on $\phi$ and the solution concentration of the data set. The temperature coefficient is positive in all cases except $\phi=1$, where it is small and negative.

It is argued that the temperature coefficient should be negative, since as temperature increases, the polyethylene lattice tends toward the hexagonal where the distinction between (110) and (200) planes disappears.

(3) Analysis in case (b).

In this case, $\phi$ has striking effects. For regime II analysis, at $\phi=I / 2, \rho$ and $\gamma$ show discontinuities. For the regime I analysis, at $\phi=0$, $\rho$ becomes infinite and $\gamma$ becomes zero. The only consistent data set are obtained at $\rho=1$. Here $\rho$ has values that range from 1.1 to 1.2 . However, $y$ has values slightly greater than unity for regime I analysis and slightly less than unity for regime II analysis.

(4) The effect of solution concentration.

The data chosen for analysis were obtained at concentrations of 0.01 percent and 0.1 percent. This change in concentration changes $T_{d}^{0}$ from $377.9 \mathrm{~K}$ to $378.5 \mathrm{~K}$ for the 0.01 percent and the 0.1 percent concentrations, respectively. The change in $r$ with concentration predicted by kinetic theory is exactly the opposite of that observed. At a constant temperature, $r$ is predicted to 
decrease with increase of concentration; the data of Blundell and Keller and Valenti and Pedemonte show it to increase. If these data are accepted as correct, the results would indicate changes in $\gamma$ and/or $\phi$ with concentration.

(5) Conclusions:

The following tentative conclusions may be drawn from the analysis

(a) $\sigma_{e}^{200}>\sigma_{e}^{110--T h e ~ f o l d ~ e n e r g y ~ f o r ~ d e p o s i t i o n ~ o f ~ s t e m s ~ o n ~ t h e ~(200) ~ f a c e ~ i s ~ o f ~ t h e ~ o r d e r ~ o f ~} 10$ percent greater than that for deposition on (110).

(b) $\sigma^{200} \approx \sigma^{110}$ - The lateral surface free energies are approximately equal. The actual relative values cannot be established until the crystallization regime question for these crystals is settled, and the theoretical question of the value of $\phi$ is answered.

(c) It is very probable that $\sigma_{e}{ }^{200}$ has a slightly different temperature dependence than $\sigma_{e}{ }^{110}$.

(d) The values of $\phi$ and $L$ are outstanding problems in the kinetic theory of polymer crystallization.

(e) The kinetic theory predicts a different concentration dependence of $r$ than that observed by Blundell and Keller and Valente and Pedemonte unless the surface energies change with concentration and the change is different for different crystal faces.

(f) While no specific analysis was carried out on the effects of molecular weight, some remarks on its effect may be made. For a given solvent and concentration, theory predicts an increase of $T_{d}^{0}$ with molecular weight. Hence, at constant temperature at a given concentration, $r$ should decrease with increase of molecular weight, absent any change of $\rho$ or $\gamma$ with molecular weight. The data in references [1] and [2] show an increase of $r$ with molecular weight. The opposite effect is found by Takamizawa et al. [3] in xylene, and by Khoury [4] in other solvents.

[1] Blunde11, D. J.; Keller, A. J. Macromol. Sci.-Phys. B 2: 337; 1968.

[2] Valenti, B. ; Pedemonte, E. La Chimica e Industria 54: 112; 1972.

[3] Takamizawa, K.; Urabe, Y.; Hara, T. Reports on Prog. in Poly. Phys. XII: 179; 1969.

[4] Khoury, F. A.; Bolz, L. H. Bul1. Am. Phys. Soc. 26, A55 (1981).

\section{The Morphology of Polyethylene Crystals Grown From Solution}

\section{F. A. Khoury and L.H. Bolz}

The lateral growth habits and nonplanar conformations of polyethylene crystals grown from dilute solutions are known to vary depending on the crystallization temperature, other factors remaining equal (solvent, mol. wt., polymer concentration).

We have previously reported that crystals exhibiting "truncated" lateral habits and having aspect ratio $r^{\star}$ in the range 1.6 to 6 were grown from heptyl acetate and dodecanol. The following effects of temperature, molecular weight, and solvent on the aspect ratio of crystals were observed. (1) As expected, for a given molecular weight, concentration, and solvent, the higher the crystallization temperature the higher $r$. In addition, whereas the crystals with low $r$ exhibited well defined (110) and (200) faces, the (200) "edges" of the lamellae tended to become curved, and all the more pronouncedly so, the larger $r$. (2) For a given crystallization temperature, concentration, and solvent, the higher the molecular weight, the smaller $r$. (3) For a given crystallization temperature, concentration, and molecular weight, $r$ was larger for crystals grown form heptyl acetate than dodecanol, which is a poorer solvent. These results clearly illustrate the strong dependence of $r$ on the undercooling $\Delta T$.

Our current investigations are aimed primarily at determining the nature of the conformations of crystals of various fractions using scanning transmission electron microscopy and selected area electron diffraction. Our earlier studies had been limited to an examination of crystals of Fraction I (Mol. wt. 11,400) grown from dodecanol at $115^{\circ} \mathrm{C}$.

These crystals had an aspect ratio $r \sim 6$. The main feature of interest was that the majority of the crystals had asymmetric conformations, i.e., the two halves of the lamellae situated on opposite sides of the long axis were not mirror images of one another. More recently, we have found this to be the case for three other species of crystals with smaller aspect ratios. These were: (1) Crystals of Fraction I, $r=3.5$ (grown from heptyl acetate at $105^{\circ} \mathrm{C}$ ); (2) Crystals of Fraction I, $r=3.2$ (grown 
from dodecanol at $112^{\circ} \mathrm{C}$ ); and (3) Crystals of Fraction III, Mol. wt. $=100,500, r=2.5$ (grown from heptyl acetate at $112^{\circ} \mathrm{C}$ ). It remains to be determined how widespread is the manifestation of this phenomenon among the crystals (of the various fractions) whose aspect ratio is in the range $r=1.6$ to 6.

Interest in asymmetric crystals such as the ones we have observed so far, stems from the not unreasonable speculation that they have features in common with, and hence will shed some light on, the nature of the experimentally less accessible constituent lamellae in axialitic and spherulitic structures formed in bulk polymer.

\section{The Energies of (110) and (200) Folds in Polyethylene Crystals}

\section{F. A. Khoury, J. Mazur, and B. M. Fanconi}

The energies of (100) and (200) folds in polyethylene crystals are of interest in connection with the elucidation of the origins of two features of the morphology of solution-grown as well as melt-grown crystals. These two features are: (1) The occurence of fold staggering resulting in the formation of hollow pyramidal, tent-like, or otherwise nonplanar lamellar crystals; and (2) the dependence of the lateral growth habits of the crystals on the crystallization conditions. The particular feature of interest in the latter context in the dependence of the aspect ratio $r$ of the lamellae on the crystallization temperature (see previous Subtask, "The Morphology of Polyethylene Crystals", F. A. Khoury and L. H. Bolz).

As an initial step in examining the possible relationships between the above mentioned morphological features and the (110) and (200) fold energies, minimum energy conformation for models of such folds were computed. Adjacent reentry was assumed for the following three cases: (1) Isolated (110) and (200) folds at the surface of a lamella; (2) (110) and (200) folds surrounded by and interacting with kindred folds situated in the same and adjoining (110) and (200) planes, respectively. In (1) and (2), the stems bridged by the folds were assumed to be at right angles to the crystal surface); and (3) (200) folds surrounded by neighboring (200) folds packed in a staggered manner, i.e., the crystal surface was assumed to be (201).

The computations of fold energies involved the summation of pair-wise interactions calculated as a function of interatomic separations $r$ for both inter and intra molecular interactions. The nonbonded carbon-carbon, carbon-hydrogen, and hydrogen-hydrogen interactions were assumed to be represented by Buckingham type potential functions $\left(E=A e^{-B r}-C / r^{6}\right)$.

Interactions for $r>0.6 \mathrm{~nm}$ were neglected. The empirical parameters (A, B, and C) of the Williams IV set were used. Additional energy contributions due to bond angle and bond stretching deformations, as well as due to rotations around backbone bonds were also computed. A generalized Urey-Bradley force field was assumed for these intramolecular interactions. The minimum energy fold confromation was determined in each case using the Newton-Raphson iterative procedure. Only the atoms in the folds were allowed to change in the energy minimization process, the coordinates of the carbon atoms in the stems underlying the folds were held fixed. The polyethylene unit cell parameters used were $a=7.4 \AA, b=4.95 \AA, c=2.536 \AA, \alpha=\beta=\gamma=90$ deg, setting angle $\theta=48$ with respect to the b-axis.

In the case of isolated folds (Case I above) the (200) fold energy was lower than that of the most favorable (110) folds by $\sim 1 \mathrm{kcal} / \mathrm{mole}$ (in close agreement with [1]). In contrast, when interfold interactions were taken into account (Case II) the (110) fold energy was $\sim 3 \mathrm{kcal} / \mathrm{mole} 10 \mathrm{wer}$ than the (200) fold energy. This difference was determined from a comparison of the most favorable lateral packing arrangement of (110) folds with that of (200) folds. Staggering of (200) folds (case III, lamellar surface (201)) results in a lowering of the fold energy. The energy corresponding to the most favorable lateral packing of staggered $(200)$ folds was $\sim 0.9 \mathrm{kcal} / \mathrm{mol}$ lower than that for unstaggered (200) folds.

It is interesting that the computations of fold energies summarized above, in which the interactions between neighboring folds are taken into account (Cases II, III), indicate that the energy of (200) folds is higher than that of (110) folds. This result of the comparison of the most energetically favorable models for each type of fold, and the results of the analysis (based on the kinetic theory of polymer crystal growth) of the changes in the lateral growth habits of polyethylene crystals grown from xylene solutions as a function of undercooling, are in qualitative agreement (see Crystal Growth Kinetics and the Lateral Growth Habits of Polyethylene Crystals, E. Passaglia and F. A. Khoury).

Calculations are at present being carried out to determine the effect of staggering on the energy of (110) folds.

[1] Corradini, P. et al. Macromolecules 4: 770; 1971. 
R. W. Penn

The objective of this project was to develop a rational technique for measuring the rates of breaking and formation of chemical bonds affecting the mechanical properties of elastomeric sealant materials aging under geothermal down-well conditions. The project was to include a theoretical and experimental approach following the treatment developed by Tobolsky, et al. as modified by Kearsley and reported in detail previously. This treatment relies upon a simple modification of the kinetic theory of rubber elasticity

$$
\lambda f_{\lambda}-\mu f_{\mu}=\operatorname{NkT}[g(\lambda)-g(\mu)]
$$

where $\lambda, \mu$ are stretches, $f_{\lambda}$ and $f_{\mu}$ are the forces acting on the faces of the prism perpendicular to stretch $\lambda$ and $\mu$ respectively, $k$ is Boltzmann's constant, $T$ is absolute temperature. $g(\lambda)$ is an arbitrary function which is to be measured by experiment. This equation is consistent with a recent phenomenological theory of Valanis and Landel if we equate their $\lambda w^{\prime}(\lambda)$ with our $N k T g(\lambda)$. In the case of simple extension, $f_{\mu}$ and $f_{\nu}$ acting on the force free sides of the sample are zero, $\mu$ and $v$ are equal to $\lambda^{-1 / 2}$ (because of the assumed constant volume) and Eq. (1) becomes

$$
\lambda f=N k T h(\lambda)
$$

where $f$ is the tensile force producing the $\operatorname{stretch} \lambda$ and $h(\lambda)$ is given by

$$
h(\lambda)=g(\lambda)-g\left(\lambda^{-1 / 2}\right)
$$

Corresponding equations can be found for other homogeneous isochoric deformations (such as biaxial extension or pure shear) giving the forces in terms of the experimentally determined function $g(\lambda)$.

When $N$, the number of segments between intermolecular bonds per unit volume, does not change with time, Eq. (1) corresponds to a perfectly elastic material which will exhibit no stress relaxation. For elastomeric materials, this might be appropriate to describe equilibrium isothermal behavior at room temperature. That is, if we look at the behavior of an ordinary rubber at room temperature stretched from the undeformed state and held for a few minutes until the modulus settles down to the "rubbery plateau", the forces required to hold it stretched will then be almost constant in time and Eq. (1) will apply. The stress relaxation occuring at shorter times (sometimes called "physical" stress relaxation to distinguish it from "chemical" stress relaxation) is presumably caused by the slipping of intermolecular entanglements and other temporary bonds. The equilibrium stress of the "rubbery plateau", however, probably reflects chemical cross-links or other, more permanent bonds between segments. With time, in an agressive atmosphere such as in geothermal down-well conditions, even these chemical crosslinks can be expected to break down and, as a result of the chemically active atmosphere new crosslinkages might be formed simultaneously. Suppose a sample of material is aged in such an atmosphere, then the quantity $N$ of $\mathrm{Eq}$. (1) will change in time reflecting the net change in crosslink density and giving rise to softening or embrittlement of the sample. Consider the following experiment. A virgin sample of elastomer with $N_{0}$ effective segments between crosslinks per unit volume is extended to a stretch, $\lambda$, and the tensile force (when it settles down to a steady value) is measured. The sample is then allowed to recover and is then aged for a fixed time, t, in some atmosphere of interest. During this time the atmosphere will cause crosslinks to break and to form or chain scission to occur so that $N$ will change to a new value, $N_{0}+n(t)-m(t)$, where $n(t)$ is the number of segments formed in time $t$ per unit volume and $m(t)$ is the number of segments destroyed. At this point, the sample is removed from the aggressive atmosphere and the measurement of simple extension is repeated in the inactive laboratory atmosphere. If the $\lambda$ and $T$ apply for each simple extension measurement, Eq. (2) applies in each case with only $f$ and $N$ varying and we can write

$$
f(t) / f_{0}=\left(N_{0}+n(t)-m(t)\right) / N_{0}
$$

where $f_{0}$ and $N_{0}$ refer to the virgin sample and $f(t), n(t), n(t)$ refer to the sample aged for time $t$. Thus, this experiemnt can be used to monitor the total change in segments per unit volume induced by the aging.

The present report covers experimental work consisting of the assembly of apparatus to simulate geothermal down-well conditions and some preliminary tests conducted on two samples of Y267 EPOM high temperature packer seal formulation (Y267-XXX11-6, and -7) which were supplied by L'Garde, Inc.

The environmental apparatus consists of a pressure vessel constructed from Hasteloy C-276. It was manufactured by LECO Corporation, Tem-Pres Division. It is rated for use up to 9000 psi at temperatures up to $400{ }^{\circ} \mathrm{C}$. It provides a working chamber two inches in diameter and ten inches long. It can be placed in an electrical resistance furnace supplied by the same company together with a temperature controller. The pressure vessel is connected to a cylinder gas source with provisions for 
purging, bleeding, etc. The tubing and valves, etc. are all high pressure stainless steel construction rated up to 10,000 psi for atmosphere not hostlle to type 303 stainless steel.

Test specimens were die cut from the $6 \mathrm{in}$. square sample. The specimens were straight strips $0.25 \mathrm{in}$. wide and $2.75 \mathrm{in.} \mathrm{long.} \mathrm{They} \mathrm{were} \mathrm{approximately} 0.080 \mathrm{in}$. thick. They were held in small simple clamps each consisting of two small bars of aluminum between which the specimen could be held by means of two steel screws. The grips were designed so that specimens could be held at constant extension in a simple slotted cylinder device which would permit up to twelve specimens to be held in the pressure chamber simultaneously. The grips could also be attached simply to test fixtures which fit our hydraulic powered test machine.

This machine is controlled by a dedicated mini-computer. A BASIC language program was modiffed to perform a stress relaxation test in uniaxial extension. The samples were loaded into the machine and stretched 10 percent. The tension in the specimens was then measured at times between 0.05 second and 100 seconds after the loading. We were thus able to cover approximately two and one half decades on a logarithmic time scale and the stresses were calculated.

Four specimens of each of the EPDM elastomers mentioned above were prepared. Their stress relaxation behavior was measured. They were then stretched by various amounts from zero to thirty percent. They were placed in the pressure chamber in dionized water. The system was then purged with nitrogen at 2000 psi four times to remove oxygen. The heater was turned on and during the time (approximately one hour) that the system was heating to $265^{\circ} \mathrm{C}$ the system was purged twice more. During these purges the water was permitted to boll. The system was held between 260 and $265^{\circ} \mathrm{C}$ for approximately one hour and then allowed to cool to room temperature. All of the specimens had failed at the clamps. Those which were unstretched showed severe crack damage in the area of the clamps. This disappointing result is probably due to the large localized biaxial strains imposed by the clamping.

The specimens were then reclamped and again tested in stress relaxation tests at 10 percent strain. The form of the stress relaxation curves had not changed but the level of stress required to maintain the strain had reduced by approximately 15 percent. There was no significant correlation of this decrease with the level of stretch. This may only indicate that they all falled early in the test and that they all spent most of the time at zero stretch. It appears that it will be necessary to redesign the specimens to avold the high strain concentrations at the clamps. This might be accomplished by the use of dog-bone shape specimens or by the use of ring shape specimens which could be die cut from sheets. This would avoid the nonuniformity problems which we experienced in working with o-ring samples. 
One of the most rapidly growing areas of high payoff polymer applications is composite materials. The addition of a reinforcement, as either particles or fibers, to polymers can dramatically improve their performance, and as a result they can be utilized in a wide range of generic applications where the requirements are beyond the capabilities of simple polymers. This expanded potential has led to increased usage in many diverse areas ranging from aerospace to automotive. Two major factors behind the expanding application of these materials are that their use is highly energy efficient and this can significantly reduce the need for critical materials such as cobalt and chromium whose limited supply is of critical concern to the national interest.

Although composite materials have great potential, the same multicomponent composition that is responsible for their high performance also gives rise to numerous problems with regard to the processing, fabrication, and prediction of fallure behavior or service life. Since the composite field is still at an early stage of development, many of these problems can be directly attributed to a lack of basic knowledge, and adequate test methods in the composites area. In response to this need, a new Task has been initiated during 1982. The objectives are to carry out long range research to provide industry and other Government agencies with test methods, material property data and failure prediction models that are essential if the full potential of these materials is to be achieved. In line with the two primary problem areas mentioned above, there are two major thrusts in this Task. The first seeks to gain a better understanding of the basic science involved in processing and fabrication of these materials while the second addresses the development of the basic science concepts, methods, and data needed to assist in improving durability and enhancing the capability for failure predictions.

During the first year Task programs have been developed to address both areas of concern. Several projects are studying cure, i.e. the chemical reactions that occur during processing and represent the most important factor in successful fabrication. Of special interest are experiments investigating rapid cure and the curing of films since these areas are of great practical importance. Another program is developing quality control techniques for raw materials, an area of great concern to industry. The failure behavior of both particle and fiber reinforced polymers is also being studied with special emphasis on the development of techniques, such as $x$-ray scattering, which can provide information about the state of the materials prior to fracture. To assist in conducting this broadly based effort three outside experts spent time working in the Division during the last year: Dr. Raganathan, an industrial research associate from Martin Marietta; Dr. James Koutsky, an Intergovernmental Personnel Act employee from the University of Illinois; and Dr. Anthony Kinloch, a foreign guest worker from the Ministry of Defense in England.

\section{Failure Behavior of Particle Reinforced Polymer Composites}

\section{L. Hunston, A. J. Kinloch ${ }^{1}$, and G. W. Bullman}

Most of the polymers used in structural application are inherently brittle and must be reinforced or toughened. One empirically developed method that has proven to be quite successful is the incorporation of small particles of a softer material in a matrix of the brittle polymer. Although composite materials of this type often have very high fracture energies their failure behavior is usually quite complex and involves large viscoelastic effects.

In an effort to gain a fundamental understanding of the failure behavior of such materials, NBS is currently studying two different model systems: a polysulfone filled with polytetrafluoroethylene particles and an epoxy with epoxy/polybutadiene particles. The first system is of interest to the Navy for certain seal applications while the second serves as a model for most commercial structural adhesives. The program focusses on developing test methods for such materials and studying the mechanisms of failure. As part of this effort a joint program was initiated in 1979 with scientists from the University of Illinois and the Ministry of Defense (Waltham Abbey Laboratory) in England ${ }^{1}$. During the last year, Dr. A. J. Kinloch from Waltham Abbey spent 3 weeks at NBS as a guest worker to help coordinate results from various parts of the program. As a result of this visit the empirical failure model developed two years ago in this program has now been extended to 4 different elastomermodified epoxy formulations. The most significant aspect of this development is that the temperature dependence for all 4 systems could be described with a single Arrhenius type activation energy whose value was identical to that obtained from yield experiments. It has previously been suggested that yielding was a crucial step in the toughening mechanism for these materials but this provides the first direct evidence for a relationship between the two properties.

[1] Hunston, D. L.; Rushford, J. L.; Wang, S. S.; Kinloch, A. J. Proceedings of the SPI Conference on Reinforced Plastics/Composites. Washington, DC; 1982, January. 
[2] B1tner, J. L. ; Rushford, J. L. ; Rose, W. S. ; Hunston, D. L. ; Rlew, C. K. J. Adheston 13: 3; 1981.

[3] Kinloch, A. J.: Shaw, S. J.: Hunston, D. L. Proceedings of the International Conference on Deformation and Fracture. Cambridge, England, 1982, March.

[4] Hunston, D. L.; Wang, S. S.; KInloch, A. J. Organic Coatings and Plastic Proprints 47; $407 ; 1982$.

\section{Interlaminar Fracture of Composites}

D. L. Hunston, G. W. Bullman, and J. A. Koutsky²

A major area of concern in the use of continuous-fiber rofnforced composftes is delamination, that is the growth of cracks botween the layers of Plbers. Delamination can substantially reduce the performance of composites in numerous ways but compressfon strength is particularly sonsitive to this type of damage. The conventional polymeric matrix resins, 1.e., the materfal that binds the flber together, are very brittle and therefore subject to damage partfcularly when loaded by 1 mpact. Th1. demage involves matrix cracks which can easfly grow into large areas of delamination. The problems this can generate in practical applications have lod to a widespread interest in developing polymeric matrix materials with Improved resistance to both inftial damage and subsequent growth of this damage into a delamination. In addition, there 1s a need to develop desion data that can be used to predict the growth rate of delamination. Unfortunately, although a number of test mothods are now boing used, fow $1 f$ any have been doveloped to the extent that they can provide the quantitative information that is needed.

In response to this problem, a jolnt program has been establlshed among resuarchers at NBS, NASA Langley, the University of IIlinols, and several composites manufacturers. As part of this program, NBS is examining various test mothods for dotermining interlaminar fracture. The tests omploy specimens with of ther rectangular or triangular geomotries. A precrack is generated between flber layers in the specimen and an Increasing load is applfed perpendicular to the precrack so that subsequent crack growth occurs between the layers of plbers. The critfcal value at which this crack growth begins 1 s noted and converted to a fracture onergy using approprlate equations.

During the last year, the program has studied the effacts of specimen size, geometry, and lay-up (the sequence of flber layers and their orfentations). Data from these tests have been correlated with the stress analysis work at the University of Illinols and the composites testing program at NASA Langley. These results have led to a serles of guldelines for the design of test specimons when the polymeric matrix is a brittle material. Future work will focus on testing composites made with toughened matrix materials and thermoplastic resins. The effects of loading rate, temperature, and environment on the fracture test will also be examined. This will help establish both the validity of the test methodology and a data base of information for model systems representing the various types of polymers.

\section{A Non-Destructive Technique to Monftor Cure of Adhesive and Composite Resins}

\section{L. Hunston and J. A. Koutsky}

In applications such as piber-reinforced composites and adhesives the polymer must be flufd in the early stages of fabrication so that the proper flow, leveling, wetting and spreading at interfaces, etc. can be achleved. The polymer must then harden by cooling, solvent loss, or chemical reactions to form a rigid material. In most cases the successful fabrication of a final product requires that assoclated changes in mechanical properties take place at the appropriate times and rates. It is desirable therefore to have nondestruetfve techniques that can monitor these changes as they occur. Unfortunately. this is gnerally very difflcult, particularly since many of the applications involve thin flims, whose mechanical properties are difficult to measure and cannot usually be predicted from experiments on bulk samples.

To address this problem, an effort is being made to develop a now method for nondestructively characterizing thin plims. During the last year an ultrasonic shear wave technique has been studied as a candidate test method. The experiment involves the propagation of a shear wave in a quartz plate coated with the thin polymer sample. By monitoring the attenuation of the wave in the strip. it is possible to follow changes that occur in the coating. The flrst samples tested were an epoxy-type polymer that cures by free radical inftiation. Figure 1 shows a plot of attenuation vs curing time for two formulations containing the same epoxy but with different concentrations of accelerator. The experiments clearly show that there is an initiation period prior to the onset of cure. The rate of cure influences the width of the transition while the ultimate attenuation level depends on the structure of the cured film among other factors. When the accelerator concentration is changed both the reaction rate and the induction period are altered but the ultimate attenuation level remains the same. This type of information can be crucial in designing epoxy-based adhesives and composites.

${ }^{2}$ University of Wisconsin 


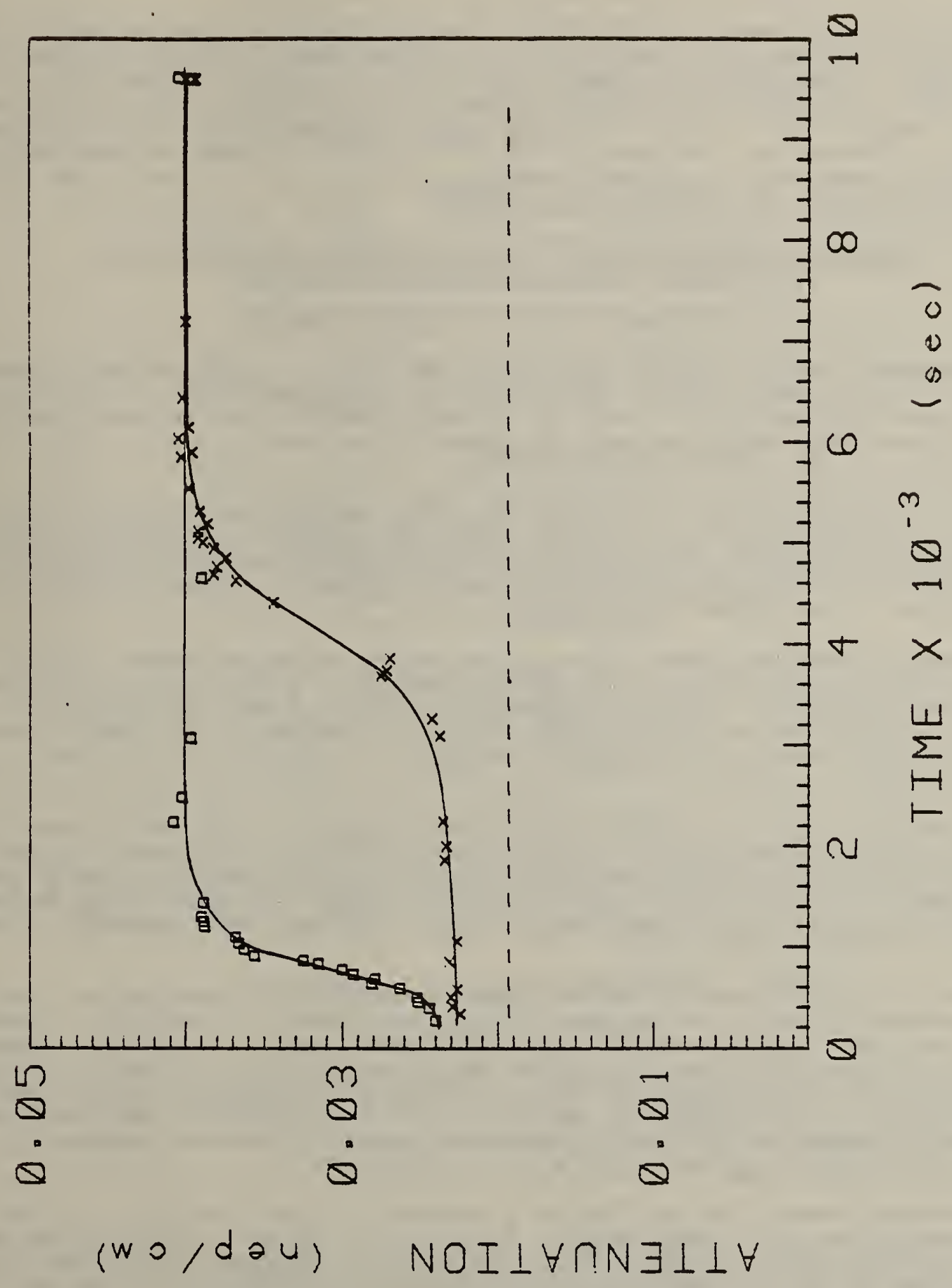

Figure 1. Cure data for the epoxy/accelerator system, dashed line for uncoated strip $x$ for 0.25 percent accelerator, and for 0.35 percent accelerator. 
The results to data indicate that this technique can be used to qualitatively monitor changes that occur during the liquid-to-solid transition in important polymer systems. During the next year an effort will be made to quantify this test. As part of this effort the measurement capability will be expanded to permit the determination of velocity as well as attenuation. In addition, the program will investigate the possibilities for coupling this technique with other methods such as dielectric tests that may be more amendable to process control measurements. The information provided by this coupling would make both measurement techniques more useful.

Measurement of Microvold and Microcracks in Composite Materials Using Small Angle X-ray Scattering Techniques

$$
\text { W. L. Wu }
$$

Small angle x-ray scattering (SAXS) has been used extensively in structural characterizations of heterogeneous systems. Most work, both theoretical and experimental, deals with systems composed of only two phases. A theoretical development treating systems of three phases (matrix, reinforcing fibers or fillers, and microcracks) has been advanced. It is shown that the volume content and the characteristic lengths of the microcracks or volds can be determined from the angular dependence of the $x$-ray scattering intensities. The analysis are valid for both deformed and undeformed specimens. Incorporating the experimental data, useful information regarding the size and content of microcracks within damaged particulate composites has been obtained.

\section{Industrial Research Associate Program on Thermal and Spectroscopic Properties of Polymer-Based Composite Materials}

B. N. Ranganathan ${ }^{3}$, J. H. Flynn, and B. M. Fanconi

Dr. Ranganathan (Martin Marietta Corporation) completed a six-month Research Program in the Polymer Science and Standards Division. During this time, extensive use was made of NBS equipment to evaluate potential test methods as a basis for the establishment of quality control procedures which promise to significantly reduce the cost of inspecting the Thermal Protection System (TPS) which insulates the External Fuel Tank for the Space Shuttle.

The TPS insulation consists of a superilight ablator composite material, approximately $1 / 2$ in. thick, that is applied directly onto the outer surface of the aluminum external tank, and, on top of the composite ablater material, approximately $3 / 4 \mathrm{in}$. thick polyurethane foam is applied for additional insulation. TPS insulation serves an important function in that it prevents ice formation, and also minimizes boil-off of the cyrogenic liquid fuels.

The composite superlight ablator layer of the thermal protection system is functionally more critical than the polyurethane layer. The composite consists of silicone resin, glass fibers, carbon black, phenolic microspheres, silica microspheres, cork, etc., of which silicone resin is the most important component for 1 t serves as the binder that holds together all the other components of the composite, and further functions as an adhesive that bonds the composite to the aluminum surface of the external fuel tank.

The present investigation is concerned with the evaluation of silicone resins. The main purpose of this research was to develop effective quality control procedures using state-of-the-art techniques that are rapid, accurate, and more rellable than the currently used methods, so that a "good" lot can be distinguished from a "bad" lot of material. This distinction of a "good" from a "bad" material is of immense importance, as it alleviates expensive repairs that are labor intensive, and results in a very cost effective external tank program.

Two types of silicone materials were evaluated as a part of this investigation. Both types are purchased commercially, and as such the exact chemical formulations are proprietory in nature. Type I silicone is fast curing (gel time--10 mins) and low strength (tensile strength approximately 50 psi), while Type II is slow curing (gel time--several hours) and high strength (tensile strength approximately 1000 ps1); the higher strength of the latter 1s due to the presence of a flller material. Both types of silicones are two component systems, and the curing mechanism is via an addition reaction between silane (component-B) and vinyl (component- $A$ ) groups; in addition, component $A$ contains a platinum catalyst. In manufacturing, an $A$ to $B$ ratio of $10: 1$ is used.

Two techniques; namely, DSC and FTIR, were evaluated for their viabllity as effective quality control procedures for the intended purpose. Evaluation of individual components was not possible using DSC; that is, no useful thermal property information was obtainable in the temperature range of interest. Therefore, DSC was used solely for studying the curing reaction. On the other hand, FTIR permitted the evaluation of individual components as well as the curing reaction.

3 Martin Marietta 
Results obtained to date indicate that FTIR is a viable quality control technique. An attempt will be made to use this technique in the future at the Martin Marietta quality control laboratory for the evaluation of polymeric raw materials prior to their use in manufacturing.

The establishment of FTIR as a viable quality control technique with its intended future applfcation and attendant benefits at Martin Marietta Aerospace is a direct result of the research associate's work at NBS.

\section{Analysis of Rapid Thermoset Resin Curing}

\section{G. A. Senich}

Thermosetting resins are distinguished from the thermoplastics by the three-dimensional nature of the chemical linkages formed in these materials during the cure process. A network of chemical bonds is formed irreversibly by heat or chemical means during curing or cross-linking processes and renders the material substantially infusible and insoluble. This is in contrast to thermoplastic materials which are capable of being repeatedly softened when heated and hardened when cooled. Articles fabricated from thermoset materials are generally insoluble, resistant to dimensfonal changes and flow, and able to withstand high temperature heating without melting.

Thermosets are considerable articles of commerce. Their usage in the U.S. has been estimated to amount to over 1.1 billion pounds in 1979. The most commercially important thermosetting resins are phenolic-, urea-, and melamine-formaldehydes, alkyds, polyurethanes, epoxies, and polyesters. These materials are commonly used as the polymeric matrix of highly filled structural composites, a market which amounted to over 2 billion pounds of fabricated material in the U.S. for 1979. Composite parts are produced by molding or laminating operations which generally require either a long time in a closed mold or press at a high temperature and pressure or a lengthy post-cure outsfde the mold, again at high temperatures. These long times for thermal curing, on the order of one to several hours, restrict the rate of production of composite articles. Rapidly curing thermoset resins could greatly reduce production time and permit a large increase in the volume of fabricated composite output.

Another area in which rapid curing is of preponderant importance is the printing process. At typical paper speeds, a thermoset polymeric ink has only a one to ten second curing period for achieving sufficient mechanical strength to allow the substrate to be wound onto a takeup roll without damage to the printed image. Natural-product based inks are now used in intaglio currency printing operations and usually provide acceptable performance. However, it is desirable to investigate rapidly curing synthetic thermosetting resins that could alleviate some of the difficulties encountered with material variability and undesirable solvents in printing with the current inks as well as others anticipated to occur with the introduction of new printing machinery.

A program has been initiated to assess the current state of rapid-cure thermoset matrix resin science and technology. Suitable example systems of interest, principally epoxies, acrylated oligomers, and unsaturated polyesters/acrylics, are being examined. Test methods for chemical or functional group characterization by FTIR, and mechanical characterization by rheometer and viscometer are being developed and coordinated to provide an understanding of how chemical reactivity and mechanical properties are related during the curing process. Novel methods for studying the kinetics of rapid curing, such as monitoring the mechanical property changes of a thin, curing film on a quartz substrate by ultrasonic shear wave propagation, are also being employed. The program will provide a better understanding of the chemistry of processing thermoset polymers and aid in the identification of possible candidate materials for special applications, such as rapidly curing synthetic thermosets suitable for use as ink vehicles, matrix resins which could increase the rate of production in the manufacture of composite articles, and repair formulations for quick mending of composite parts.

\section{Degree of Polymerization of Thermoset Resins Used in Composites}

\section{W. L. Wu and B. M. Fanconi}

Physical properties as well as the resistance to adverse environments of thermoset resins depend strongly on the degree of polymerization (state of cure). The curing process of thermoset resins is believed to be incomplete if the curing temperature is well below the glass transition temperature ( ${ }_{g}^{\infty}$ ) of the polymers in their completely cured states. This situation is commonly encountered in practical cases.

A FTIR technique was developed for monitoring the degree of cure and was applied to assess the degree of polymerization of commonly used thermoset resin systems. Cure characteristics were examined as a function of type and amount of initiators and accelerators, as well as the flexibility of the prepolymer. It has been found that only a limited amount of polymerization occured after the vitrification points. The degree of polymerization is mainly controlled by the viscosity of the monomer instead of the type or amount of the initiators. 
Polymeric materials find many high payoff applications in construction, transportation, and electrical systems where long-term durability is essential to competitive performance. The growing use of polymeric materials in these generic applications, generates need for long-term research aimed at providing a scientific basis for accelerated tests of service iffe. In fact, a recent report by the National Research Council states, "The lack of adequate measurement techniques for predicting the service life of new materials and components is a major barrier to the acceptance of polymeric materials."

To develop reliable methods for predicting the service life of polymeric materials, we need to identify the scientific basis of (1) the rates and mechanisms by which polymeric materials degrade under the appropriate environmental conditions and (2) the rates and extents of the migration of protective additives from polymeric materials. The collection and interpretation of these two types of information have been the main effort in this task.

Research this year on degradation mechanisms and rates includes investigations of the hydrolysis of polyesters and the use of thermo-gravimetry to measure the rates and activation energies of the thermal degradation of several polymers. In addition, we have initiated a collaborative program with the Building Materials Division to investigate the kinetics and mechanism of the photodegradation of PMMA, a polymer widely used for solar cover plates.

Almost all useful polymeric materials have low-molecular-welght substances incorporated in them to develop or maintain desirable properties. In use, these substances inevitably diffuse through the polymer to some extent and may migrate out of the polymer entirely. The polymer is then left with poorer properties or made more vulnerable to degradation and failure. This is a major failure mode of polymers, and lifetime prediction methods must explicitly include the rates of additive diffusion along with the rates of the inhibited and uninhibited degradation reactions. The number of commercially usable polymer-additive combinations is too large to be considered efficiently on a case-by-case basis. The emphasis in this task is, therefore, on the development of general principles or models of diffusion that are applicable to the widest range of generically important systems. In addition, knowledge of the general principles which control diffusion through solid polymers is important to the design and evaluation of improved additive systems. Work this year on the migration of additives includes measurements of diffusion rates by solvent extraction and by inverse chromatography and investigations of slow diffusion in solid polymers and concentrated polymer solutions by microfiuorimetry and by a photobleaching technique.

\section{Hydrolytic Degradation of Polyester Polyurethane Elastomers}

\section{W. Brown, R. E. Lowry, and L. E. Smith}

Polyester polyurethane elastomers are useful specialty polymers. Unfortunately, their ester linkages are subject to acid-catalyzed hydrolysis. Earlier, we investigated this process and developed kinetic schemes that can be used to predict lifetimes of these polymers, with and without carbodimide stabilizers.

\section{In this year we investigated several additional aspects of this hydrolytic degradation.}

Published data indicate that cross-linked polyester polyurethanes in magnetic tape binder may appear stable to hydrolysis at about 25 percent relative humidity, RH, because of the equilibrium between ester hydrolysis and esterification [1]. This equilibrium ought to be general for ester hydrolysis and so may affect estimates of hydrolytic stability in other polyester systems. We determined the equilibrium acid contents, $\left[A_{e}\right]$, in three polyester diols and in soluble poly(butylene adipate) and poly(caprolactone)-based polyurethanes. Values of $\left[A_{0}\right]^{-1}$ are about $7 \times 10^{-4}$ mol/g at 25 percent $\mathrm{RH}$. Molecular weight of the polyurethanes equals $\left[\mathrm{A}_{\mathrm{e}}\right]^{-1}, \mathrm{e}^{\mathrm{about}} 1400$, if there is one acid group per molecule. The equilibrated polymers are weak, brittle, solids. Presumably the initial crosslinks or perhaps reaction of acid with iron oxide made the polyurethane appear more stable on the equilibrated tape.

Another study concerned the behavior of carbodiimide stabilizers. We had observed that polyester polyurethanes consumed carbodiimide stabilizers from three to ten times faster during hydrolytic aging than did the polyester diols used to make the polymers. We made a poly(caprolactone) diurethane diol from a 2:1 mixture of diol and diisocyante. This material consumed carbodiimide during hydrolytic aging about as slowly as the polycaprolactone diol. Thus, the difference in rates of carbodiimide consumption was not explained but is not associated with the presence of urethane in the polymer. 
A third study was made of the effect of the initial acid content on the rate of consumption of carbodilmide. We used the same number of moles excess carbodiimide over acid in two polybutylene adipate diols that differed in initial acid contents by a factor of 20 . The diol having the higher initial acid content consumed the excess carbodiimide faster during hydrolytic aging. Presumably this was due to hydrolysis of the acyl area produced by reaction of acid and carbodifmide. At lower initial acid contents this reaction apparently is not important because uncatalyzed hydrolysis of ester also consumes carbodifimide. The result indicates the practical importance of using low acid diols in making polyurethanes, even if a stabilizer is to be used.

[1] Cuddihy, E. F. IEEE Trans. Mag. 16: 558; 1980.

\section{Prediction of the Long-Term Stability of Polyester-Based Recording Media}

D. W. Brown, R. E. Lowry, and L. E. Smith

PET is the base material in many information storage and retrieval systems. We are investigating methods of estimating the useful lifetime of PET in archival storage, under sponsorship of the National Archives. Hydrolysis of the ester linkage is the most important cause of degradation. Various samples are being aged under a variety of conditions. Samples have been examined after aging at $55,85,100$, and $115{ }^{\circ} \mathrm{C}$ at 25 to 100 percent RH. Gel permeation chromatograms were used to calculate molecular weights. They indicate that about one scission/ original molecule $\left(5 \times 10^{-5} \mathrm{~mol} \mathrm{scission} / \mathrm{g} \mathrm{polymer}\right)$ makes the polymer quite brittle. The imprecision in molecular weight is such that scissions probably are uncertain by 1 to $2 \times 10^{-5} \mathrm{~mol} / \mathrm{g}$. Thus, the gel chromatograph, which consumes only about $5 \mathrm{mg}$ of PET per determination, does not appear to measure chain scissions with enough precision to anticipate embrittlement. Efforts will be made to improve the precision of this measurement.

Acid contents were measured by potentiometric titration. The change in acid content is about equal to the number of scissions, as is expected for hydrolysis of a polyester. Acid contents are somewhat more precisely known than molecular weights but measurement of the former requires about $2 \mathrm{~g}$ of polymer. This is too much for use as a monitoring technique.

Attempts will be made to develop infrared and sonic methods of analysis.

Examination of Processes in Thermogravimetry

\section{B. Dickens}

Calculated vapor pressures have been used [1] in a first-approximation model of the vapor above thermally degrading polyethylene [2]. The results suggest that the "normal boiling" of large molecules in the range $C_{30}$ to $>C_{90}$ forces the rapid growth of bubbles in polyethylene degrading at low pressures, and the bubbles grow to significant size because the liquid is relatively elastic. The trend of decreasing apparent overall activation energy with increasing pressure is ascribed to contributions of low latent heats of vaporization from molecules up to $\sim C_{30}$, depending on the imposed pressure. The trends of vapor composition for various temperatures and pressures have been studied. In general, both the temperature and the pressure should be as low as possible to minimize the contribution of the latent heats of vaporization to the overall activation energy and interference from bubbles in the molten degrading polymer.

Table 1 gives the activation energies found [2] for thermally degrading polyethylene, together with the associated pressures, temperatures, and apparent orders of reaction (calculated over small changes in extent of reaction, $\sim 5$ percent, at extents of reaction greater than 40 percent). The activation energies were determined using the factor-jump method of thermogravimetry, which avoids the need to specify a form for the dependence of the rate of weight loss on the extent of reaction and also determines the activation of energy without any correlation with the preexponential factor of the Arrhenius equation. Such activation energies are expected to be relatively unbiased.

The apparent overall activation energy and the apparent order of reaction decrease as the pressure increases (table 1), which suggests that simple evaporation [3] is a significant factor. Evaporation of liquid alkanes such as $\mathrm{C}_{36} \mathrm{H}_{74}$ is easily shown experimentally to be of zero order, depending only on the available surface area, when the vapor pressure of the pure liquid is below the external pressure. When the vapor pressure is equal to the external pressure, normal boiling occurs in that the temperature of the liquid depends on the applied pressure and ideally cannot be increased by increasing the heating rate. Such increases provide more vapor, and when the quiescent surface area is not sufficient to allow the required amount of vapor to form, more surface area is provided through the creation of bubbles. 


\begin{tabular}{|c|c|c|c|c|}
\hline \multicolumn{5}{|c|}{$\begin{array}{ccc}\text { pressure, } & \mathrm{mm} \mathrm{Hg} \text { of } & \mathrm{N}_{2}+\text { volatiles } \\
\sim 0.005 & 8 & 800\end{array}$} \\
\hline E (kcal/mole) & $65.4 \pm$ & \pm 0.5 & $64.8 \pm 0.3$ & $62.6 \pm 0.5$ \\
\hline temperature ${ }^{a},{ }^{\circ} \mathrm{C}$ & 425 & & 445 & 460 \\
\hline bubbles? & yes & & no & no \\
\hline order of reaction & $1.5 \pm$ & 0.5 & $0.8 \pm 0.3$ & $0.7 \pm 0.3$ \\
\hline
\end{tabular}

\footnotetext{
a For roughly equal rates of weight loss from approximately the same size of sample at roughly the same extent of degradation at the specified pressure.
}

In vacuum conditions, unsteadiness in the sample weight is observed and has been attributed to bursting bubbles. No shocks to the balance are observed at overall pressures of 8 and $800 \mathrm{~mm} \mathrm{Hg}$ of $\mathrm{N}_{2}$. The calculations show that normal boiling ceases at $\sim \mathrm{C}_{29}$ for $8 \mathrm{~mm} \mathrm{Hg}$ and $445{ }^{\circ} \mathrm{C}$, at $\sim \mathrm{C}_{8}$ for $800 \mathrm{~mm} \mathrm{Hg}$ and $460{ }^{\circ} \mathrm{C}$, and at $\sim \mathrm{C}_{85}$ for $0.005 \mathrm{~mm} \mathrm{Hg}$ and $425{ }^{\circ} \mathrm{C}$ (see table 1) for the hypothetical mixture of hydrocarbons. Thus the vacuum case requires that molecules up to $C_{B s}$ be forced rapidly out of the liquid. The slowness of diffusion for the largest of these molecules $\left(C_{30}\right.$ to $C_{85}$ were not under boiling conditions at $8 \mathrm{~mm} \mathrm{Hg}$ and $445{ }^{\circ} \mathrm{C}$ ) to the quiescent surface must require the formation of "local surface" in the form of bubbles, so that they may be volatilized. Because most nucleation is heterogeneous, these bubbles would form on the wall of the sample cup. The smaller molecules will have very large vapor pressures at the temperatures of interest and are so unstable with respect to boiling that they are able to enter even very small bubbles. Thus the small molecules will act as a dissolved permanent gas. In this sense, the smaller molecules "nucleate" the bubbles which then accept the larger molecules as the bubble size increases.

The temperatures in table 1 correspond to degradation rates of $\sim 1: 4: 10$ when the observed [1] activation energy of $65.4 \mathrm{kcal} / \mathrm{mole}$ is applied. These temperatures are for approximately equivalent rates of weight-loss under the pressures specifled in table 1 . Since the weight loss is independent of whether methylene units are joined or not, this means that, for equal overall rates of weight loss, samples which are degrading faster because of their higher temperature are volatilizing shorter molecules. In particular, the average molecular weights in the vapor phase are $1: 1 / 4: 1 / 10=1: 0.25: 0.1$ at these temperatures.

The calculated average number of carbon atoms per volatilizing molecule is 49:20:9 for the three cases considered. These values lie in the ratio 1:0.41:0.18. The last two are in approximately the correct ratio to each other. The first, for the near vacuum case, is somewhat too low, as expected because the tail obviously should include molecules longer than $C_{100}$, but these molecules were not included in the calculations because their Antoine coefficients are not avallable. Also, the total pressure was probably not exactly the $0.005 \mathrm{~mm} \mathrm{Hg}$ assumed for the vacuum case and the upper 1 imit of bolling molecules is very sensitive to the total pressure in this region. A lower pressure would give a higher average molecular weight and a greater range of boiling molecules.

The important aspect is to minimize the tail representing "normal evaporation" as opposed to "normal bolling", and the calculations show that the appropriate conditions are that the pressure should be as low as possible. The temperature has less effect in reducing the proportion of weight loss by evaporation but has a large effect on the size of molecules volatilized and hence on the propensity for explosive bubble formation. The results also indicate the extent to which the assumption of a sharp cut-off in the size of molecules volatilized is realistic. The largest molecule evaporating during degradation in vacuum was assumed to have 70 carbon atoms in the Wall-simha treatment [4,5]. Suehiro and 0 'Shima [6] estimated an upper limit of $\sim 26$ carbon atoms for evaporation in an inert atmosphere at $420^{\circ} \mathrm{C}$. This upper 1 imit is indeed dependent on the imposed pressure and is really a distribution. The cut-off is sharpest at very low pressures, and changes markedly with temperature. Also, as Wall et al. remarked [4], it may be expected to vary with extent of degradation. The dependence on temperature and degradation probably explains much of the discrepancy in degradation products reported for polyethylene.

[1] Dickens, B. Thermochim. Acta 55: 217; 1982.

[2] Dickens, B. J. Poly. Sci. 20: 1065; 1982. 
[3] Carman, P. C. Trans. Faraday Soc. 44: 529; 1948.

[4] Wa11, L. A.; Madorsky, S. L.; Brown, D. W.; Simha, R. J. Am. Chem. Soc. 76: 3430; 1954.

[5] Simha, R.; Wall, L. A. J. Poly. Sci. 6: 39; 1951.

[6] Suehiro, T.; 0'Shima, E. Kobunshi Ronbunshu 34: 241; 1977.

\section{Thermogravimetric Studies of Polymer Degradation}

\section{B. Dickens}

Thermogravimetry is often and widely used to study polymers because it can be applied to specimens in any condensed form. In a continuing series of investigations, we have used the highly automated factor-jump method of thermogravimetry, developed at NBS, to study the thermal degradation of polyisobutylene (PIBu).

The overall activation energy, $E_{a}$, of the thermal degradation of PIBu has been measured to be 49.2 $\pm 0.3 \mathrm{kcal} / \mathrm{mole}(207 \pm 1 \mathrm{~kJ} / \mathrm{mole})$ over the range 365 to $405{ }^{\circ} \mathrm{C}$ in $\mathrm{N}_{2}$ at $800 \mathrm{~mm} \mathrm{Hg}$ pressure and flowing at $4 \mathrm{~mm} / \mathrm{s}$ over the sample. This is consistent with some values reported for thermal degradation in vacuum and in solution. In $5 \mathrm{~mm} \mathrm{Hg}$, an apparent activation energy of $52.1 \pm 0.5 \mathrm{kcal} / \mathrm{mole}(218 \pm 2$ $\mathrm{kJ} / \mathrm{mole}$ ) was found and, in vacuum, a value of $57 \pm 3 \mathrm{kcal} / \mathrm{mole}$ (238 $\pm 13 \mathrm{~kJ} / \mathrm{mole})$. Troublesome bubbling made the vacuum values difficult to measure.

Substitution of reasonable values for the activation energies of initiation, $E_{i}$, and termination, $E_{t}$, and the activation energy, $E_{a}$, for vacuum degradation in the equation $E_{a}=E_{i} / 2^{i}+E_{d} E_{t} / 2, y i e l d s$ an activation energy $E_{d}=20 \mathrm{kcal} / m o l e$ for the unzipping reaction. This equation presupposes a degradation mechanism of random initiation, unzipping, and biomolecular termination. Substitution of reasonable values of $\Delta H$, the heat of polymerization, in the definition $\Delta E=E_{p}-E_{d} s u g g e s t s$ that the activation energy of polymerization reaction at $375{ }^{\circ} \mathrm{C}$ is approximately $7 \mathrm{kcal} / \mathrm{mole}$.

\section{Variable Heating Rate Thermogravimetry}

\section{J. H. Flynn}

The prediction of the lifetime of a polymer by extrapolation from weight-loss data requires a thorough analysis of the degradation kinetics both in the initial phases and over a wide temperature range. Techniques have been developed in which entire kinetic spectra are compared among experiments performed at heating rates from $6 \%$ min to $9 \%$ day. Simple methods of plotting data from these techniques have been used for diagnosing shifts in reaction mechanism, uncoupling of competing processes, and testing the validity of kinetic models.

Considerable weight-loss in vacuum, nitrogen, or air is found to occur below $200{ }^{\circ} \mathrm{C}$ when toluene-2,4-diisocyanate (TDI) and diphenyl methane-4,4'-diisocyanate (MDI), polyether and polyester soft segment, 1,4-butanediol polyurethanes are heated at very slow rates. Weight-loss in vacuum is punctuated with bursts of volatiles as the internal pressure of the dissociated diisocyanate builds up more rapidly than it can dissipate by diffusion. TDI polyurethanes degrade at lower temperatures and with lower activiation energies than do MDI polymers due to their faster diffusivity and greater volatility. These factors suggest that techniques may be developed for the direct recovery and reuse of di isocyanates from polyurethane wastes.

\section{Photolytic and Thermal Degradation of Poly(Methyl Methacrylate)}

\section{B. Dickens}

A study on degradation in solar cover plate materials is being carried out in collaboration with D. Waksman and J. W. Martin of the NBS Center for Building Technology. PMMA was selected as an appropriate material because it is inexpensive, relatively resistant to weathering, and noncrystalline. Approximately $1 \mathrm{in.}$ square specimens of $1 / 16$ in. thick plates of UV-absorber free PMMA were exposed to five different temperatures and four levels of xenon arc radiation filtered through borosilicate glass. Nearly all published studies on photodegradation of PMMA used $254 \mathrm{~nm}$ radiation, which is readily absorbed by the ester groups of PMMA. This study used only wavelengths greater than $300 \mathrm{~nm}$ to simulate degradation by solar radiation.

Mechanical properties are well known to be influenced by changes in molecular weight. In particular, reduction in the molecular weight of PMMA causes embrittlement (once a critical level is reached) and lowering of the glass transition temperature. Molecular weight distributions were therefore determined for more than 250 specimens (including triplicates and controls) using gel permeation chromatography. Computer programs which allow data collection and data processing with 
considerable precision were written by J. D. Bernes end F. L. McCreckin of the Polymer Science end Stendards Division.

In addition to analysing the effect of the degradation on the whole specimen, we meesured the molecular wight distribution as function of depth along the Irradiation direction. Microtoming was unsatisfactory because the material was too brittle. Mililing wes satisfactory way of providing more than 20 slices, provided the specimen was large enough. Because of the small size of most specimens, we flrst sawed selected specimens into up to 5 slices with diamond wafer saw. GPC analysis of these slices has shown approximately equal degradation on the front and back sides of the PMMA sheets and considerably less in the center, probably due to oxygen starvation there. Quantitative analysis of chain scissions in terms of degradation temperature and incident flux is now beginning.

UV spectra are being used to Investigate chromophores which are formed and consumed during the degradation. Shifts in the edges of the UV absorption bands in PMMA are being studied using difference spectra on whole and on sliced specimens. The bands appear to correlate with the extents of thermal and photolytic degradation.

Water analysis using FTIR to study the $5263 \mathrm{~cm}^{-1}$ IR absorption of ater combination band has shown that PMMA is extremely sensitive to the humidity of the atmosphere and typically contains 1 to 2 percent water. The effects of such levels of water on the degradation mechanism through plasticization of the PMMA will be studled next.

Fluorescence Measurement of Ant1oxidant Migration from Low Density Polyethylene into 1-propanol

F. W. Wang and B. F. Howell

Although the fluorescence technique has been used to determine additive concentrations in polymers $[1,2]$, to our knowledge, there has been no report on the use of this technique to measure the diffusion of an additive from a polymer matrix into an extracting solvent. To carry out successfully a diffusion measurement by the fluorescence technique, one must take precautions to exclude oxygen from the extracting solvent since oxygen quenches the fluorescence of the additive and, in some cases, contributes to 1 ts photodegradation. We describe here how oxygen exclusion was incorporated into a procedure to measure the diffusion of an additfve from a polymer matrix into an extracting solvent. We also describe the application of this procedure to the diffusion of an amine antioxidant, $N$, $N$ 'diphenyl-p-phenylene-diamine (DPPD), from low-density polyethylene (LDPE) into 1-propanol and we give a preliminary value for the diffusion coefficlent of DPPD in LDPE under the experimental conditions described below.

\section{Experimental}

\section{DPPD-LDPE F11ms}

DPPD was purffied by recrystallization from toluene. NBS-SRM 1476, a low-dens1ty polyethylene whole polymer, was washed under a nitrogen atmosphere, first with toluene at $110{ }^{\circ} \mathrm{C}$, and then with heptane at $22^{\circ} \mathrm{C}$. The DPPD-LDPE powder, In which 0.05 wt. \% of DPPD was uniformly distributed, was pressed in air into pellets with an IR pellet press. These pellets were placed in teflon molds, fused under a nitrogen atmosphere, and then quenched with dry 1ce. The films thus obtained were approximately $0.355 \mathrm{~mm}$ thick; they were trimmed with an arch punch to form discs $0.95 \mathrm{~cm} 1 \mathrm{n}$ diameter. Two $f 11 \mathrm{~ms}$ were a combined mass of $0.49 \pm 0.006 \mathrm{~g}$ were extracted during each diffusion coefficient determination.

\section{Extraction Procedure}

Ten $m l$ of 1-propanol were added to a welghed, specially constructed extraction vessel (fig. 1). A side arm of the vessel (A in $f 1 \mathrm{~g}$. 1) was attached with wax to a high vacuum 11 ne. Solvent in chamber $D$ was degassed inside the vessel by repeated freezing, evacuation and thawing. Partial loosening of the stopcock (E In $\mathrm{flg}$. 1) opened the vessel to the vacuum 11 ne while sealing it from the atmosphere. By means of the vacuum 11ne, oxygenfree, purfiled nitrogen, at a pressure slightly greater than one atmosphere, was introduced into the vessel. Then, the vessel stopcock was removed. Nitrogen gas flowed through the vessel whlle plastic tubes were first inserted; then DPPD-LDPE P1lms were 1ntroduced into the vessel. The plastic tubes were designed to prevent transfer of stopcock grease into the vessel, and to guide the flims into the empty vessel chamber ( $\mathrm{fn} f 1 \mathrm{~g} .1$ ). After f1lm 1ntroduction, the stopcock was quickly replaced, and the 1-propanol allowed to thaw. The nitrogen-f1lled vessel was removed from the vacuum line with the stopcock closed, and the system equilibrated to $22{ }^{\circ} \mathrm{C}$.

At the beginning of an extraction experiment, 1-propanol was introduced into the chamber containing the fllms by tipping the vessel. The fluorescence intensity of the solution in cell $B$ was observed to increase with time. The intensity was measured at sultable time intervals until no further increase was noted, usually after a week. Between measurements, the vessel was placed in a constant temperature bath at $22^{\circ} \mathrm{C}$, and the alcohol solution was stirred with a magnetic stirrer. To guard 
against stopcock oxygen leakage, nitrogen was passed through needles piercing a serum cap covering the open arm ( $A$ in fig. 1) above the stopcock.

After completion of the $22{ }^{\circ} \mathrm{C}$ experiments, control experiments were performed, which established that no additional DPPD was extracted when the system temperature was increased to $60^{\circ} \mathrm{C}$.

\section{Results and Conclusion}

To evaluate the diffusion coefficient $D$ from measurements of the increasing fluorescence of the 1-propanol solution, fluorescence intensity was plotted as a function of square root of extraction time. For these plots, there was an initial linear increase in fluorescence intensity followed by approach to a horizontal asymptote. From these plots, we obtained the initial slope, and the asymptotic fluorescence intensity, $F_{\infty}$. Use of the relation [3]

$$
M_{t} / M_{\infty}=4 / \pi^{1 / 2}\left(D t / e^{2}\right)^{1 / 2}
$$

(for which $\ell$ is the thickness of polymer film undergoing extraction, $t$ is the time in seconds, and $M_{t}$ and $M_{\infty}$ are the amounts of diffusing substance present in the extracting solvent at times $t$ and at equilibrium respectively) allowed calculation of $D$ if the ratio $M_{t} / M_{\infty}$ was replaced by the ratio of sample fluorescence intensity at time $t, F_{t}$, to sample fluorescence intensity after extraction was complete, $F_{\infty}$. We verified that in the concentration range of these experiments, fluorescence intensity is linearly related to concentration.

Five independent measurements give a value for $D$ of $8.5 \times 10^{-10} \mathrm{~cm}^{2} / \mathrm{s}$ with a standard deviation of $0.6 \times 10^{-10} \mathrm{~cm}^{2} / \mathrm{s}$ for migration of DPPD through LDPE when extracted with a 1-propanol at $22{ }^{\circ} \mathrm{C}$.

[1] Kirkbright, G.; Narayanaswamy, R.; West, T. Anal. Chim. Acta 52: 237; 1970.

[2] Crompton, T. Additive migration from plastics into food. Pergamon Press: New York; 1979, p. 47.

[3] Crank, J. Mathematics of diffusion. Oxford Press: London; 1956, p. 248.

\section{Diffusion of Small Molecules in Polymers}

\section{S. S. Chang, J. R. Maurey, and W. J. Pummer}

In FY82, besides finishing up the experiments with the migration of $n-\mathrm{C}_{18} \mathrm{H}_{38}, \mathrm{n}_{-} \mathrm{C}_{32} \mathrm{H}_{66}$ and 3,5-di-t-butyl-4-hydroxytolune (BHT) from linear and branched polyethylenes, isotactic polypropylene, and (ethylene-vinyl acetate) copolymer into various solvents (NBSIR 82-2472), the following areas of research were undertaken.

Migration of BHT from Impact Modified Polystyrene (IMPS). The rubbery IMPS swells and loses dimensional rigidity by the action of many commonly used fat or oil simulants. Therefore, in choosing a simulant, one should consider the degree of swelling or the retention of the dimensional rigidity. The study also indicated the somewhat limited utility of IMPS in contact with certain types of food.

Migration of Styrene Monomer from Polystyrene. The migration of carbon-14 1 abeled styrene monomer from so-called "crystal styrene" occurs in the glassy state below the T. The kinetics of diffusion in the glassy state sometimes follows the Case II behavior, such that the initial amount migrated is linearly proportional to time, $t$, rather than to $t^{12}$ as in the normal. Fickian behavior. The diffusion coefficient in the glassy state is generally several orders of magnitude less than that in the rubbery state.

Absorption of small molecules from Migrant Solutions by Polymers. The migration of small molecules across the polymer/solvent interface may also be studied by the absorption of the migrant by a polymer from the migrant solution. If the absorption is to be monitored by the decrease of the migrant concentration in the solution, then the range of the partition coefficient and the solubility are required to be within certain narrow limits, in order to generate changes that are large enough for a meaningful kinetic study. We have already performed such a study in the case of absorption of $n-\mathrm{C}_{18} \mathrm{H}_{38}$ by polyethylenes from a 50 percent ethanol solution. Currently the absorption of $n-\mathrm{C}_{32} \mathrm{H}_{66}$ by polyethylenes from an ethanol solution is under study.

\section{Solubilities of n-Alkanes in Polyolefins and in Various Solvents}

\section{S. S. Chang and J. R. Maurey}

Solubilities of oligomers of linear polyethylene, such as $n-C_{18} H_{38}$ and $n-C_{32} H_{66}$, in various solvents, as well as in polyolefins, have been determined. The ratios of solubilities in the solvent versus that in the polymer agree well with the partition coefficients as observed by both extraction and absorption experiments reaching equilibrium distribution. 
The solubilities of $n$-paraffins in $n$-heptane behave rather ideally. In the ideal case, the solubility as a function of temperature may be predicted from the heat of fusion and the melting points of the solute.

Long chain n-paraffins exist in a rotator phase just a few degrees below the melting point. Solubility measurements on the low temperature phase lead to a hypothetical melting point slightly above the rotator phase transition temperature.

Measurements of Relative Diffusion Constants in Polymers

G. A. Senich

Relative diffusion constants for oligomers, stabilizers, and other low molecular weight compounds in polymers have been obtained by inverse phase gas chromatography (IGC) experiments. This method of polymer analysis provides a convenient way of studying the physical chemistry of interactions occuring between polymers and volatile organic materials or probes. The methodology is very similar to conventional gas chromatography, the principle difference being that a polymer of interest replaces the typical stationary phases used to sorb the vapor-phase probes. The experiment involves injecting the probe and an inert marker into a flowing carrier gas stream which propels the substances through a column containing the polymer, coated on an inert support packed within the column. The time lag between injection and elution of the vapor peak and its width or standard deviation characterize the polymer-probe interactions, which are strongly influenced by the carrier gas flow velocity. The steady-state probe distribution reached between vapor and polymer phases at low flow rates allows polymer-probe thermodynamics to be studied. A steady-state distribution is not reached at high flow rates where the kinetics of polymer-probe interactions are accessible and the probe diffusivity in the polymer can be obtained as described below.

Chromatographic peak broadening is commonly quantified by the height equivalent to a theoretical plate, which can be calculated from the standard deviation and, ideally, is proportional to the carrier gas flow velocity at high velocities. This constant of proportionality, $c$, is related to the diffusivity of the probe in the polymer, $D$, by the following expression:

$$
D=2 d^{2} k /\left[3 C(1+k)^{2}\right]=Y k /\left[C(1+k)^{2}\right]
$$

where $d$ is the thickness of the polymer layer and $k$ is the column capacity factor derived from the time lag. If $C$ and $k$ are determined by IGC experiments and $d$ is known from the geometry of the polymer in the column, the polymer-probe diffusion coefficient can be obtained from the equation. Several problems have been identified in determining the diffusivity in this manner. The equation applies only to peak spreading in localized areas of the column, although the average velocity is often used in its application. Gas phase diffusion can also contribute to peak spreading and, in turn, to the $c$ value found at high carrier flow rates. In addition, any calculation of $D$ is very sensitive to the distance over which diffusion occurs, $d$. The value of $d$ used in the equation can vary widely, depending on whether the polymer is distributed as a thin, uniform film or in much thicker regions, although previous studies [1,2] have indicated that the latter geometry is favored. These complications make absolute diffusivities difficult to determine by IGC, however, if several probes are studied in the same column, the diffusivity of one probe in the polymer can be compared to that of another irrespective of the method of calculating the absolute diffusivity. Such IGC experiments have been conducted; the $C$ and $k$ values for selected alkane probes are given in the table below. This information can be used to obtain relative diffusivities by splitting the equation into two terms: the first describing the polymer geometry only and the second characterizing the polymer-probe interactions. The first term is constant in all determinations with a particular column and is abbreviated as $Y$. This form of the equation was used to determine the probe diffusivity values given in column four of the table. By selecting one of the probes as a reference, in this case octadecane, the diffusivity of a probe in the polymer relative to the diffusivity of the reference can be obtained from a ratio of the respective results, also given in the table. An absolute diffusion constant for each probe in the polymer can be obtained from the relative diffusivity values if an absolute diffusion coefficient for any of the probes in that polymer exists. Using the previous value of $2.4 \times 107 \mathrm{~cm}^{2} / \mathrm{s}$ for octadecane in HPDE [2], the absolute diffusivities indicated can be obtained by multiplying the relative diffusion coefficients by this factor.

Relative and absolute diffusion coefficients can be utilized in a large number of industrial applications, such as in modeling the controlled release of bioactive substances, estimating the amounts of oligomers, plasticizers, and antioxidants migrating or leached from polymers over time, and designing membrane separation processes.

[1] Senich, G. A. CHEMTECH 11: 360; 1981.

[2] Senich, G. A. Polymer Preprints, 22: 343; 1981. 
Relative Diffusivity of Alkanes in HDPE at $150{ }^{\circ} \mathrm{C}$

\begin{tabular}{lcrccc}
\hline Probe $^{\mathrm{a}}$ & $\mathrm{C}(\mathrm{ms})$ & $\mathrm{k}$ & $\mathrm{D}_{\text {Probe }}$ & $\mathrm{D}_{\text {Probe }}{ }^{1 \mathrm{D} \mathrm{C}_{18}}$ & $\mathrm{D}\left(10^{7} \mathrm{~cm}^{2} / \mathrm{s}\right)$ \\
\hline $\mathrm{C}_{18}$ & 21.9 & 119. & $0.38 \mathrm{Y}$ & 1. & 2.4 \\
$\mathrm{C}_{16}$ & 7.8 & 60.9 & $2.05 \mathrm{Y}$ & 5.4 & 13. \\
$\mathrm{MeC}_{15}$ & 8.7 & 49.0 & $2.25 \mathrm{Y}$ & 6.0 & 14. \\
$\mathrm{Me}_{7} \mathrm{C}_{9}$ & 18. & 12.8 & $3.74 \mathrm{Y}$ & 9.9 & 24. \\
$\mathrm{C}_{13}$ & 12. & 12.1 & $5.74 \mathrm{Y}$ & 15.2 & 36. \\
\hline
\end{tabular}

${ }^{a} C_{18}=$ octadecane; $C_{16}=$ hexadecane; $M C_{15}=2$-methylpentadecane; $M_{7} C_{9}=$ $2,2,4,4,6,8,8$-heptamethylnonane; $C_{13}=$ tridecane.

\section{Measurement of Antioxidant Diffusion by Microfluorimetry}

\section{F. W. Wang and B. F. Howe 11}

The diffusion of the antioxidant $N, N^{\prime}-\beta$-dinaphthyl-p-phenylene-diamine (DNPD) in branched polyethylene (PE) SRM 1476 is being measured by microfluorimetry according to the procedure briefly described as follows.

Two thin rectangloids of $P E$, one of which contains 0.002 percent $w / w$ of DNPD, are placed side by side in a close fitting Teflon frame, with the thin sides adjacent and in contact. The combination is sandwiched between two pieces of microscope cover glass, compressed with a spring, and heated in an evacuated chamber. After melting, followed by quenching, the DNPD concentration approximates a step function which broadens in time with diffusion at a specified temperature. Concentration profiles are measured with a microfluorimeter to yield the diffusion coefficient D of DNPD in PE.

Experiments at $70^{\circ} \mathrm{C}$ led to a reasonable value of $D$ for DNPD in PE. However, because of the limited solubility of DNPD in PE, aggregation of DNPD often took place in places corresponding to the plateau region of the concentration profile. To overcome this difficulty, we have (1) reduced the concentration of DNPD, (2) made certain that the DNPD-containing PE film has uniform antioxidant distribution, and (3) modified the film fusion procedure so as to produce a film of uniform thickness. We anticipate that more accurate values of $D$ can be obtained because of these procedural improvements. If this is not the case, we plan to use a polymer in which DNPD is more soluble than it is in PE.

\section{Measurement of Translational Diffusion of Polymers by Fluorescence Photobleaching Recovery Technique}

\section{F. W. Wang and W. H. Grant}

The diffusion coeffcient of chain molecules is a dynamical parameter which should provide a test of both scaling [1] and reptation [2] concepts proposed by de Gennes. Recently Hervet and co-workers [3] used the forced Rayleigh scattering technique to measure the diffusion coefficient (D) of polystyrene chains in benzene. They found that in the semidilute regime where the chains overlap, $D$ decreases with polymer concentration, $c$, as $c 1.7 \pm 0.1$, in agreement with scaling law predictions. However, they pointed out that more experiments are necessary to check the molecular weight dependence of the diffusion coefficient.

In collaboration with Professor En-Shinn Wu of the University of Maryland (Baltimore County), we have initiated a project to measure by the fluorescence photobleaching recovery (FPR) technique the diffusion of chain molecules in the semidilute regime. In the FPR technique, one uses high intensity illumination to deplete by photobleaching the observed volume element of chromophore-labeled chain molecules. After the bleaching pulse, the diffusion of unbleached chromophore-labeled chain molecules into the bleached volume element is observed with low intensity illumination. From the time constant for the recovery of the original fluorescence intensity, the diffusion coefficient can be obtained. The FPR technique can be used to measure diffusion coefficients in the range $10^{-11}$ to $10^{-6} \mathrm{~cm}^{2} / \mathrm{s}[4]$.

We have synthesized a chromophorelabeled polystyrene of narrow molecular weight distribution and measured the diffusion coefficients of this polymer in solutions of unlabeled polystyrene polymers. These experiments showed that the labeled polymer has the appropriate type and extent of labeling for the FPR technique. However, we were unable to obtain accurate diffusion coefficients because the geometry of the bleached volume element was not adequately controlled. To overcome this difficulty, we have designed and tested a three-compartment cell shown in Figure 1 . It has an identical 1 ight path for all the compartments. With this cell, the diffusion coefficients of the samples in two compartments can be precisely measured relative to the diffusion coeffcient of a standard sample in the third compartment. The absolute diffusion coefficients can then be determined once the absolute diffusion coefficient of the standard sample is determined by an independent method. This novel cell 


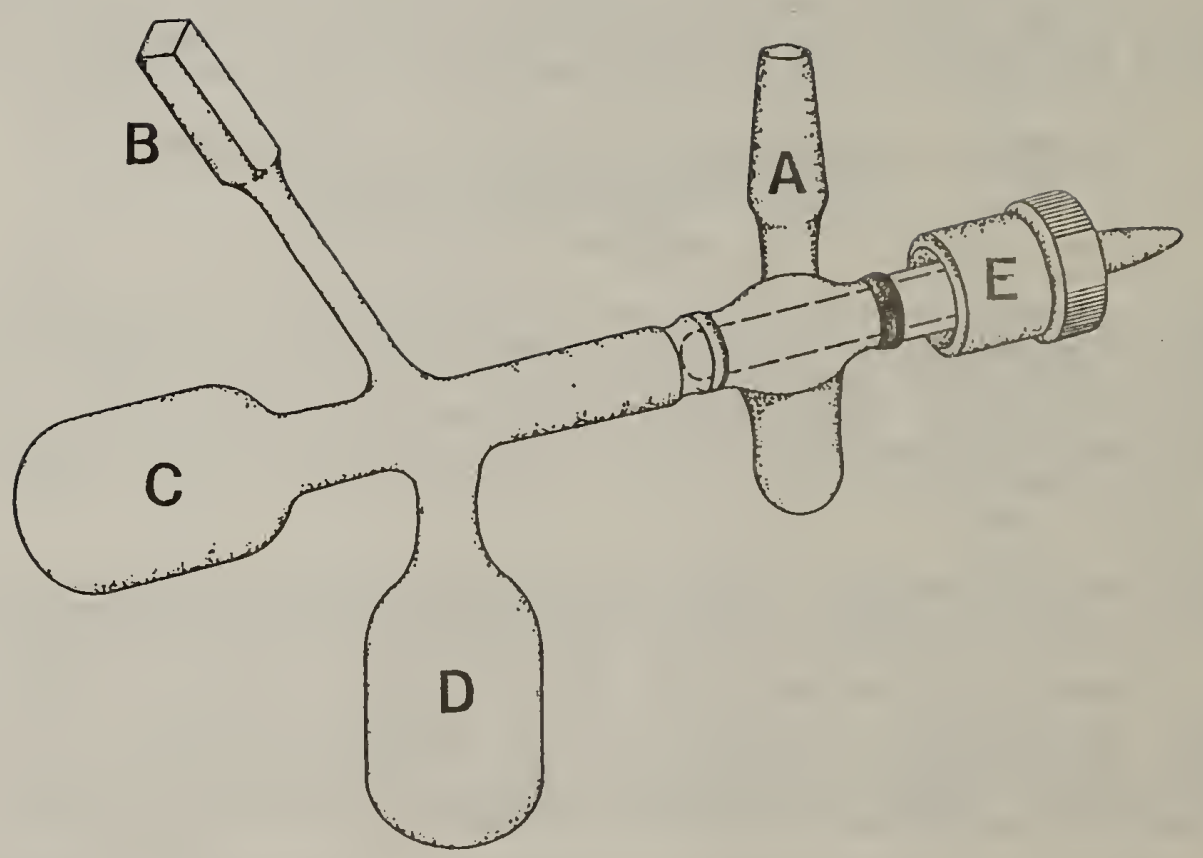

Figure 1. Extraction vessel: A, ground glass joint for connection to vacuum line; $B$, fluorescence cuvet; $C$, chamber into which films are introduced prior to extraction; $C$, chamber into which films are introduced prior to extraction; $D$, chamber in which solvent is degassed; $E$, outer part of removable stopcock. 
will be used to measure the diffusion coefficients of this and other similarly prepared polymers to check the molecular weight dependence of the diffusion coefficient in the semidilute regime.

[1] de Gennes, P. G. Macromolecules 9: 594; 1976.

[2] de Gennes, P. G. J. Chem. Phys. 55: 572; 1971.

[3] Hervet, H.; Leyer, L. ; Rondelez, F. Phys. Rev. Lett. 42: 1681; 1979.

[4] Koppe1, D. E.; Axelrod, D.; Schlessinger, J.; Elson, E. L.; Webb, W. W. Biophys. J. 16: 1315; 1976.

\section{Partition Coefficient of Vinyl Chloride in Poiy(vinyl Chloride)}

\section{S. S. Chang and W. J. Pummer}

Poly(vinyl chloride) (PVC) is one of the most widely used polymers. Its monomer, vinyl chloride also known as VCM, is a recognized carcinogen. The allowable level of VCM in the working atmosphere is regulated by OSHA and EPA. A small amount of VCM is retained by the PVC resin during the polymerization process. By steam stripping, the amount of the residual VCM in the resin may be reduced to less than $1 \mathrm{ppm}$.

Partitioning and the solubility of VCM in PVC has been studied by several authors at VCM concentrations generally exceeding $10 \mathrm{ppm}$. As the residual VCM contents in PVC, especially those being used in contact with food, may be made much less than 1 ppm. The study of the Henry's law constant should be extended to the lowest concentrations detectable.

Head-space gas chromatography provides sensitive detection of VCM without serious interferences. The detection limit is about $50 \mathrm{pg}$ with flame-ionization detector. With electron-capture detector and nitrous oxide enhancement, the detection limit may be further lowered.

We have used both manual and automatic injections for the study of the partition coefficient of VCM in PVC down to a concentration about $20 \mathrm{ppb}$. As the lowest concentration, the partition coefficient observed is still within the order of magnitude as that observed previously for high concentrations.

Further refinements are required to determine more precisely the partition coefficients at low concentrations by reducing the effects of absorption of VCM by Teflon syringe inserts and teflon septum liner, and by estimating more quantitatively the amount sampled in automatic injection operation.

\section{Self-Diffusion in n-alkane Crystals}

\section{B. L. Farmer ${ }^{1}$ and R. K. Eby}

Energy calculations for the self-diffusion of n-alkane molecules within the crystal (via vacancy migration) have been carried out for crystals with structures representative of orthorhombic and triclinic n-alkanes and monoclinic polyethylene. It is found that the energy barriers to such diffusion are generally small, and can be especially low for the latter two structural environments. There is a marked dependence of the barrier on the crystallographic direction of the diffusion, both for different lateral directions as well as for the longitudinal direction. While the barriers to motion are fairly small, a significant portion of the activation energy for self-diffusion is the energy for the creation of the vacancy. The latter energy is dependent on the model envisioned for that process. Overall, the total calculated energies (for various models) are in good qualitative agreement with the experimental data available. The results suggest that self-diffusion may play an important role in explaining some experimental observations for crystals of n-alkanes. 


\section{G. T. Davis}

Task Leader

NBS research in the electrical properties of polymers encompases a broad range of generic applications from insulating materials with low loss to electronically conducting polymers. Trends in dielectric applications of polymers include higher working voltages in transmission lines to conserve energy and materials, save space, and reduce weight. Among the potential applications for the recently discovered conducting polymers are light weight solid state batteries, electrochemical sensors, and energy conversion. Also included in electrical properties are the piezoelectric and pyroelectric response of polymers which have led to new types of transducers and new measurements. The goal of the task on electrical properties is to carry out long range research to provide the basis for new or improved measurements and provide concepts and data regarding the performance of polymers in high payoff electrical applications. Data, recommendations, and basic scientific concepts resulting from this work go directly to funding organizations through written reports, to manufacturers and users through research associates who work with us, personal communication, laboratory visits, and publication in archival journals.

The study of electrical conduction of organic polymers is a major new frontier in solid state physics. Most current research involves an empirical search for improved dopant-polymer combinations from a bewildering array of compounds. Chemical structure-electrical property correlations are not yet evident. The key need is for a better knowledge of the molecular packing, dopant locations, chemical changes with doping, molecular orientation, and charge carrier pathways to form a basis for modeling and predicting electrical properties. This work is ideally suited to NBS because it involves long range research on improved measuremenis, standards, test methods, and scientific concepts. Good progress has been made in an appreciable initial effort on electronic conduction.

Applications of polarized polymers as pyro- and piezoelectric transducers are rapidly growing. Transducer manufacturers as well as private and Government transducer users (DOD, EPA, HHS) need reliable basic information connecting polymer structure and polymer performance to the design of improved transducers. This work provides the scientific basis for transducer material and device manufacturers to design and develop commercial polymer transducers for use in a wide range of generic applications.

Important scientific opportunities of this work include breakthroughs in understanding the basic factors in electrical failure of polymers and discovery of novel measurement techniques which are becoming possible with newly developed polymers. For example, on-going work will show how polymers interact with electronic and ionic charges and how structure modification alters piezoelectric and semiconducting properties.

Fast ion transport in polymers provides a basis for a large and growing class of important applications including production of chlorine and caustic from brine, hydrogen production from low-head hydroelectric or ocean thermal energy sources, solid electrolytes for fuel cells and batteries, gas sensors, materials separation membranes, and electrochemical devices. NBS work is aimed at characterizing the polymer structures, measuring their properties, and relating the structures and properties to provide a scientific basis for predicting specifically and transport mechanisms at the molecular level.

The execution of the task was assisted by $\mathrm{Dr}$. C. Hansen, an industrial research associate from G.E., Dr. G. Harris, a guest worker from the FDA, and by a joint project with Bell Laboratories.

\section{Automated Dielectric Measurement}

\section{F. I. Mopsik}

This activity was begun to develop an efficient, rapid measurement system to characterize dielectric materials. It will allow material studies that were not previously feasible due either to the excessive time and equipment required or the lack of accuracy available.

The instrument being developed will record the transient electric charge response of a dielectric sample to a step change in the applied voltage in real time. This response will be transformed into the dielectric constant and loss over the frequency range of $1 \mathrm{mHz}$ to $10 \mathrm{kHz}$ and plotted by computer.

During FY82 the first version of the system has become operational and its limits examined. As the electronic circuits were completed last year, the only work done was the improvement of the generator to remove its lack of reproducibility at very short times. Improved amplifiers have improved the setting time behavior by a factor of four.

The major work this year has been to develop the transform techniques and the operating programs needed to have a fully operational system. A numerical procedure has been founded and developed that 
has a basic accuracy of better than 0.1 percent of full scale and can be carried out over 8 decades in frequency in only 1.5 minutes. In addition, a complete set of operating programs has been completed so that measurements to $10^{4} \mathrm{~Hz}$ can now be carried out in less than two hours. A series of measurements as a function of temperature can be completed in less than a week.

Initial measurements have shown that the instrument is capable of detecting a loss peak with a tan $\delta$ as low as $10^{-4}$ from a standard capacitor as well as the loss peak in polypropylene at $10 \mathrm{~Hz}$.

An invited talk at the Gordon Conference on Dielectrics on August 3, 1982 is being presented on the measurement scheme as the latest in the state-of-the-art.

\title{
Dielectric Loss Spectra of Polymers in the Microwave Region
}

\author{
A. J. Bur
}

This work was undertaken as a result of a direct request from the U.S. Army Civil Engineering Research Laboratory. It consists of a review of dielectric loss data for polymers at microwave frequencies and an illucidation of the molecular mechanisms which are responsible for microwave absorption.

A review of the literature reveals that most of the dielectric data for polymers in the frequency range $100 \mathrm{MHz}$ to $100 \mathrm{GHz}$ has been published in the past 15 years. The impetus for this work comes from the communications industry which seeks low loss materials. Well-known low loss dielectrics such as polyethylene, polypropylene, polytetrafluoroethylene, and polystyrene have been measured by several laboratories. Data for other polymers are more difficult to obtain and in some cases do not exist. One of the largest sources of data has been reports from the Massachusetts Institute of Technology, Laboratory for Insulation Research.

The molecular mechanisms responsible for microwave losses are dipoles which are part of a short segment of the main polymer chain or a side chain. A dipole in the amorphous phase is more likely to produce microwave absorption than is one in the crystalline phase.

This work is being assembled as a review and a final report to the Army.

Resistivity Measurements on Ultra-Drawn

Poly(vinylidene fluoride) (PVDF) Films

A. J. Bur

This subtask directly supports the optimization of piezoelectric polymers for applications in industry and Government. It concerns the definition of PVDF properties prior to poling which, after poling, will yield highly active transducers with optimum ageing characteristics.

Thick specimens were prepared by molding PVDF pellets in a compression mold under vacuum. Subsequent preparation steps included annealing or no annealing and various amounts of mechanical drawing ranging from $4: 1$ to $7: 1$ draw ratio at $140{ }^{\circ} \mathrm{C}$.

For resistivity measurements, the samples were fitted with guarded electrodes in order to eliminate the effect of surface currents. Several step voltages in the range $75 \mathrm{~V}$ to $275 \mathrm{~V}$ were applied to each sample and the current was detected using an electrometer or operational amplifier. The current at 10 minutes was used to calculate the resistivity.

$X$-ray measurements were also made on samples having a range of draw ratios. With these observations we were able to calculate the proportionate amount of $\alpha$ and $\beta$ phase crystals in the samples.

The results of the resistivity measurements show two effects: first, the resistivity decreases by a factor of two or foir as the unoriented samples are oriented to a 4:1 natural draw ratio; second, the resistivity increases monotonically by over a factor of 10 as draw ratio increases from 4:1 to $7: 1$. The $x$-ray measurements show that the resistivity increases as the $\beta$ phase content increases which indicates that the $\beta$ phase content increases with draw ratio.

The data reflect the polarization response of the sample to a step voltage. The current is interpreted as that due to a dispersion with a long relaxation time whose origin is dipolar orientation and/or ionic conduction in a Maxwell-Wagner process.

Samples with the highest resistivity are those with the highest draw ratio and high $\beta$ phase content. We suggest conductivity in these samples is minimized because of the presence of the polar $\beta$ phase crystals which impede the motion of conducting ions in their neighborhood. These samples are being considered as candidates for optimum transducer activity because high poling fields can be used to polarize the samples and because extremely long relaxation times will inhibit anv decrease in activity. 


\section{H. P. R. Frederikse, C. K. Chiang, and W. R. Hosier}

Polymers in which electrical conduction approaches that of metals is a new area of materials science in which there are many unsolved problems but the potential applications are enormous. NBS is making measurements on some of these systems to define the measurement problems for the purpose of improving measurements and developing test methods as well as developing concepts for the conduction mechanism in these materials.

Most of the polymers of interest are not conductive in the as-polymerized state but must be modified. The addition of oxidizing or reducing agents to develop conductivity has been referred to historically as "doping" but this term is probably inappropriate because of the large quantities of added material and the specific oxidation-reduction reactions that occur. At NBS, emphasis has been placed on poly-p-phenylene sulfide, PPS, and polyacetylene, $(\mathrm{CH})_{x}$.

Iodine changes the conductivity of PPS from $10^{-14}$ to $\sim 10^{-4} \mathrm{~s} / \mathrm{m}$ at room temperature, but this conductivity decays with time. Apparently, iodine only affects the surface of the PPS film in the early stages of the doping process. For longer periods of time this dopant strongly attacks the polymer and destroys its conductivity as well as its molecular structure. AsF 5 higher and more permanent conductivity in PPS as long as the polymer is kept below $330 \mathrm{~K}$. Measurements between 110 and $330 \mathrm{~K}$ yield reproducible values; plots of $\mathrm{In} \alpha$ vs $1 / \mathrm{T}$ indicate activation energies of 0.09 to 0.14 eV. Optical absorption of pure PPS shows a sharp cutoff at $362 \mathrm{~nm}$ or $3.43 \mathrm{eV}$ which probably represents the intrinsic gap between energy bands associated with small $\pi$-overlap of the sulfur $3 p$ and carbon $2 p$ electronic states.

Polyacetylene can be made conductive electrochemically which is easily followed by monitoring electrical current and potential. The first phase of this program is to develop the electrochemical doping and modification techniques. This is done via collaboration with E. Blubaugh and W. Yep in Dr. R. Durst's electrochemical group in the Division of Organic Analytical Chemistry. In this year, we have produced polyacetylene electrodes on platinum substrates and in freestanding form. Currentvoltage characteristics of these electrodes were measured. For a freestanding $(\mathrm{CH})_{x}$ electrode in $0.5 \mathrm{M}$ percent LiAsF $_{6}$ propylene carbonate solution, the positive potential at ( $\mathrm{CH}_{x}$ electrode attracts AsF 6 toward the $(\mathrm{CH})_{x}$ film and dopes the film. The doping reaction can be written as

$$
(\mathrm{CH})_{x}+y x\left(\mathrm{AsF}_{6}\right)^{-} \rightarrow\left[\mathrm{CH}\left(\mathrm{AsF}_{6}\right)_{y}\right]_{x}+\mathrm{yx} \mathrm{e}^{-}
$$

The extra charges were removed at the electrode through the external circuit. The doping current increased monotonically as the applied potential increased up to 2.0 volts. On the reducing of applied potential, the electrochemical current showed a peak at 0.4 volt. This suggests that the dopants are electrochemically bound to the polyacetylene. A similar response was seen in the graphite intercalation. By continuing to apply a positive potential, we can dope $(\mathrm{CH})_{x}$ to a highly conducting state.

The electrochemical response of highly doped polyacetylene is very much like a metal. This electrochemical modification of polyacetylene requires much longer time $(\sim 10 \mathrm{~h})$ than that by gas doping (ca. $10 \mathrm{~min}$ ). Conducting polymers produced in this manner may have very unique physical properties.

Since conductivity is rapidly destroyed by oxygen, the oxidation mechanism of polyacetylene and its kinetic parameters are of interest. Free standing polyacetylene films have been prepared and oxidized in air and in pure oxygen gas at room temperature and at higher temperatures. Infrared spectra of these oxidized samples were measured. An absorption between 3650 and $3200 \mathrm{~cm}^{-1}$ was not present in pure polyacetylene and is due to the steady formation of -OH groups. In most cases of hydrocarbon and polymer oxidation, there is a significant proportion of - $00 \mathrm{H}$ groups (hydro peroxide), which also absorb in this range. The absorption persisted after the sample was heated to $200{ }^{\circ} \mathrm{C}$ and thus is assigned to hydroxyl groups. Other indicators of the course of the oxidation are the absorptions at 1725 and $1670 \mathrm{~cm}^{-1}$. A general change in the background was observed. Detailed analysis is in progress.

$\mathrm{C}^{13}$ NMR spectra were taken of the polyacetylene films oxidized at $50{ }^{\circ} \mathrm{C}$ for $40 \mathrm{~h}$ in air. Heating at high temperature first converted $(\mathrm{CH})_{x}$ films from cis-to trans-form. New resonances appear at shifts of $\sim 58 \mathrm{ppm}, \sim 78 \mathrm{ppm}$, and $197 \mathrm{ppm}$. Preliminary assignment of these resonances are: the 58 ppm and $78 \mathrm{ppm}$ resonances are due to $\mathrm{sp}^{3}$ type carbons and the $197 \mathrm{ppm}$ resonance is due to $\mathrm{sp}^{2}$ type carbons in carbonyl groups. Detailed consideration of these assignments is also in progress.

The weight increase of polyacetylene due to oxidation was also studied. A sample exposed to air at room temperature for 153 days may increase in weight by over 60 percent. The electrical conductivity decreased to the order of $1 \times 10^{-12} \mathrm{~S} / \mathrm{m}$ during oxidation. 
G. T. Davis, M. G. Broadhurst, J. Lovinger ${ }^{1}$, and T. Furukawa ${ }^{2}$

The objectives of this task are to study the molecular motions, structural changes, and electrical properties associated with the process whereby polymers are made piezoelectric. Information gained from these studies will be used to optimize piezoelectric and pyroelectric response with respect to both initial activity and long-term stability.

While piezoelectric and pyroelectric activity from polymers is comparable to that from good ceramic materials, other properties of polymers often make them the preferred transducer material. Some of the polymer properties which make them so useful include toughness, flexibility, low density, broad band response, close acoustic impedance match to water, and ease of fabrication. Requests for information from other Government agencies and from industries have remained numerous. Interest in this area of research continues at a high level, as evidenced by the 16 papers on this topic presented at the American Physical Society meeting in March 1982, numerous review articles in the literature, and commercial advertisements for polymer transducers in trade journals.

The results of an investigation into the structural changes which occur in a copolymer of vinylidene fluoride and trifluoroethylene (52.8 percent VDF) upon poling and subsequent depoling at elevated temperatures were reported in a series of two publications. The work was a collaborative effort between personnel of the Polymer Science and Standards Division and the Bell Laboratories. An initial mixture of two crystal phases is converted to a single polar phase in which the chain conformation is all-trans. Upon heating to $85^{\circ} \mathrm{C}$, the polymer chains undergo a gradual transition to a somewhat disordered (trans gauche) 3 conformation which has no net polarization. Additional compositions of such copolymers $(65 / 35,73 / 27$, and $78 / 22)$ have been obtained and their properties are now being studied, again in collaboration with Bell Laboratories and Dr. Furukawa at the Institute of Physical and Chemical Research, Saitame, Japan who had been a guest worker at Bell during the previous effort. These copolymers crystallize almost completely in the all-trans conformation but do undergo a phase change to the nonpolar helical conformation at temperatures that get closer to the melting point of the copolymer as the VDF content increases. A study of the properties associated with a ferroelectric Curie point of these polymers may help in understanding why the PVDF homopolymer begins to lose piezoelectric properties at temperatures above $90{ }^{\circ} \mathrm{C}$ even though no definite phase changes have been observed prior to the melting of the polymer.

\section{Polarization and Space Charge Studies}

\section{A. S. DeReggi, F. I. Mopsik, and A. J. Bur}

A measurement method for determining the distribution of space charge or polarization within a dielectric film is now being employed. The method of imparting a thermal pulse to one surface of the sample and following the transient charge or voltage response as the temperature equilibrates was introduced to NBS by a quest worker (Dr. R. E. Collins from Australia). Recent advances in data acquisition and numerical analysis permits the determination of up to ten Fourier coefficients of the polarization or charge distribution. Details of the analysis have been submitted to the Journal of Applied Physics.

The measurement of polarization and space charge distributions in solid polymers is basic to understanding polymer-charge interactions. In the ferroelectric polymers, these interactions give rise to nonuniform polarization distributions across the thickness of film samples. In the insulating polymers, these interactions are believed to play a key role in the insulating properties, electrical strength and other performance and aging properties. Furthermore, charging phenomena are of basic interest to spacecraft designers concerned with the possibly deleterious effects of space radiation on the spacecraft-borne instruments.

The method has been applied to the study of PVDF when poled at room temperature. The regions of the film near the surfaces were shown to require higher poling fields than the bulk of the film and to remain less poled by approximately 15 percent at fields to $2 \mathrm{MV} / \mathrm{cm}$. When a film was first poled to 2 $\mathrm{MV} / \mathrm{cm}$ and reverse poled to $-1 \mathrm{MV} / \mathrm{cm}$, the center was reverse poled but the surfaces still retained polarization in the original direction with nearly zero mean polarization for the entire sample. A paper on these results has been prepared and has been submitted to the Journal of Applied Physics for publication.

A study has also been done with Professor Sidney Lang of Ben Gurion University. PVDF was poled at low fields at room temperature with $A 1$ and $A u$ electrodes to see if work function changes in the electrode materials would influence the poling behavior. Previous work indicated that this could be so. A detailed series of measurements showed that this was not the case but that apparent changes were

${ }^{1}$ Bell Laboratories

${ }^{2}$ Institute of Physical and Chemical Research, Japan 
due to the presence of a small amount of permanent polarization in the original, nominally unpolarized film. The strength of this preexisting polarization varied randomly among samples. Also, the two surfaces of the film were found not to be equivalent. These results reinforce and amplify our previous results that the film is intrinsically nonuniform in its poling behavior. A paper on these results is now in preparation.

Measurements of the polarization distribution in electron-beam charged PVC have proven interesting in that they have suggested a new and potentially useful method of measuring the electric field inside the foil during irradiation. During irradiation, the beam-injected charge is known to give rise to strong electric fields in the polymer. The polymer is believed to become poled under the action of these strong fields, even though the polymer is below its glass transition temperature of approximately $70{ }^{\circ} \mathrm{C}$. Becasue PVC is a polar material with a linear polarization vs field relation, the distribution of polarizat ion is expected to be proportional to the electric field distribution during irradiation. It turns out that this polarization is stable in time while the injected charge is neutralized or leaves the polymer within a period of a week. We believe that the measurement of the polarization distribution of an electron-beam irradiated PVC sample after it has discharged for a week under short-circuit conditions yields the electric field distribution which existed during irradiation. Thermal pulse measurements performed so far have shown stable polarization distributions with the qualitative appearance of the calculated electric field distribution during irradiation. Related measurements on PVC samples subjected to known applied voltages have shown that the polymer can be poled below its glass transition, and that the polarizability is of the expected magnitude based on the measured polarization in the irradiated samples and the theoretically predicted field. A manuscript describing this is under preparation.

\section{Spontaneous Polarization in Ferroelectric Polymers}

\section{A. S. DeReggi, S. C. Roth, G. T. Davis, and M. G. Broadhurst}

A method for measuring the pyroelectric response of polymers as a function of temperature while the temperature is increased linearly with time has been developed at NBS. The technique provides a rapid means of determining the temperature at which polarization begins to decay and is being used to establish the effects of poling variables and polymer morphology on the relative stability of piezoelectric and pyroelectric properties.

Measurements of the sample polarization temperature dependence have been performed on PVDF and on its copolymer with tetrafluoroethylene (TFE). In both cases the polarization has been found to go to zero when melting of the sample is complete, rather than at some transition temperature distinct from the melting temperature. Measurements such as those described here must be done with well characterized samples since the ferroelectric polymers, like other semicrystalline polymers, may exhibit relaxation or melting behavior which depend on the prior mechanical and thermal history. The extended range of temperature over which melting may occur, and the multiphase nature of PVDF samples also must be considered in making the connection between the sample polarization and the spontaneous polarization.

The measurements on biaxially oriented PVDF poled at $100^{\circ} \mathrm{C}$ show a gradual loss of sample polarization starting at $70^{\circ} \mathrm{C}$ and extending throughout the remainder of the semicrystalline range which finally melts at approximately $170{ }^{\circ} \mathrm{C}$. There is no evidence of ferroelectric to nonferroelectric transition as reported by others, and no sign of impending precipitous polarization decrease of the kind shown by analogous spontaneous magnetization of a ferromagnet near a Curie point. A gradual phase conversion from a polar to an antipolar phase is being considered as an explanation for the gradual loss of sample polarization. The role of defect motion in the conversion and of relaxation of orientation is also being considered as related mechanisms.

Future measurements are planned on the VDF-trifluoroethylene copolymer at high VDF content. A structural-ferroelectric transition distinct from melting is observed in this system at a temperature which depends on composition and possibly on history. Extrapolation of the transition temperature vs composition data to 100 percent VDF would place the transition in PVDF in the area of its melting point. Future measurements also are planned for poly(vinyl fluoride) which is a semicrystalline system with a single crystalline phase, in which transformation to alternate phases should not exist.

\section{Fabrication of Thick Piezoelectric Polymer Film}

\section{A. J. Bur, M. G. Broadhurst, and A. K. Tsao}

This subtask results from a direct request from the Navy to explore fabrication of thick piezoelectric polymer film for hydrophone use. Thick film is needed because the voltage response of thin PVDF films is less than typical ceramic hydrophones. The voltage response is inversely proportional to capacitance and hence directly proportional to the thickness of the film. To make the voltage sensitivity of polymers better than that of ceramics the thickness should be at least $750 \mu m$. To process and polarize a film this thick requires special equipment and so far only a couple of European companies have been successful at producing it. In this work, NBS is developing techniques whereby industrial laboratories in the U.S. can process suitable films. 
U1tra-drawn PVDF is material which has been stretched beyond its natural draw ratio of $4: 1$. Using a PVDF sample which has been previously stretched to its natural draw ratio of $4: 1$, it is possible to obtain draw ratios of 6.5 or 7.0 to 1.0 by reclamping the sample in the Instron machine and stretching at $140{ }^{\circ} \mathrm{C}$ at a rate of $0.05 \mathrm{in./min}$. During the ultra-stretch, the load increases while the sample dimensions decrease, indicating that the modulus increases with increasing draw ratio. The ultra-drawn material is desired because it contains $\beta$ phase material which poles at lower fields and has a larger polarization than other phases, it is highly oriented, and it is able to withstand high poling fields.

Poling these thick samples requires high voltages. For example, to pole a $0.3 \mathrm{~mm}$ thick sample at $2.0 \mathrm{MV} / \mathrm{cm}, 60 \mathrm{kV}$ are needed. Working at these voltages, it is necessary to suppress corona and flashover conduction by carrying out the poling in oil and to construct the sample electrodes so that high electric fields are not present at the edges.

Ten out of fourteen samples with thicknesses ranging from $0.21 \mathrm{~mm}$ to $0.53 \mathrm{~mm}$ were successfully poled. These samples had pyroelectric coefficients ranging from 2.1 to $4.1 \mathrm{nC} / \mathrm{cm}^{2} \mathrm{~K}$ and hydrostatic piezoelectric coefficients ranging from 8.6 to $15.6 \mathrm{pC} / \mathrm{N}$.

\section{Acoustic Sensors}

\section{A. S. DeReggi and S. C. Roth}

Measurements employing piezoelectric PVDF transducers in hydrophones and biomedical sensors have shown the polymeric transducers have superior performance characteristics over previously available piezoelectric ceramic devices in terms of a wide operating bandwidth and negligible insertion effects. These favorable characteristics stem from the similarity in acoustic impedance between the PVDF and the common transmission and test media used in ultrasonics, such as water, oils and, in the case of biomedical ultrasonics, body tissue. There is currently a growing interest in polymer transducers in the ultrasonic community in general and in the biomedical community in particular. The collaboration between NBS and a guest worker from the Bureau of Radiological Health (BRH) has continued during the past year. The principal aims of the collaborative work are the development of polymeric acoustical probes for measuring the radiation pattern of biomedical transducers and the development of improved methods of characterizing the performance of existing biomedical transducers using the new polymeric probes. The knowledge obtainable with the new polymeric sensors can be used to ensure both the safety and adequacy of ultrasonic dosage as well as to advance research in ultrasonic treatment.

A variety of ultrasonic probing hydrophones and hydrophone arrays have been constructed, all featuring the spotpoled, tensioned membrane design configuration which was introduced at NBS several years ago. A patent application which was filed by BRH (November 1980), is still pending, and NTIS has publicized this patent application among ultrasonic manufacturers who might be interested in its commercialization.

In collaboration with NBS, members of the CNR Institute of Clinical Physiology at the University of Pisa are evaluating piezoelectric sensors for the monitoring of cardiac patients. Preliminary clinical data on peripheral pulse signals obtained at Pisa with NBS sensors have been presented at the Computers in Cardiology Conference in Florence in September 1981. These data appear to give useful information about cardiac function, and although this information is complementary to, it is not necessarily contained in EKGs.

\section{Fast Ion Transport in Solid State Polymers}

\section{K. Chiang, G. T. Davis, A. J. Bur, and A. S. DeReggi}

Fast ion transport in solids involves a highly specific interaction between the ionic species and the matrix resulting in transport which is orders of magnitude greater than simple diffusion. Alkali halide complexes with polyethylene oxide show this unique property for cation movement. With financial support from the Office of Naval Research, personnel experienced in electrical properties have recently begun a long range investigation into the relation between the structure and morphology of such polymers and their ability to transport particular ions. These types of polymers have potential high payoff applications as solid state electrolytes for batteries, highly specific electrochemical sensors, and fuel cell separators.

\section{Evaluation of Cellulose Acetate as a Neutron Dosimeter}

\section{G. Broadhurst, A. J. Bur, and R. B. Schwartz}

This problem resulted from a request from the Department of Energy to evaluate a published report that the electrical resistivity of cellulose acetate was sensitive to the amount of neutron radiation which it has received. If true, this effect could be very useful as a basis for a personal neutron disometer. 
Several films of cellulose diacetate and triacetate were irradiated with a fluence of about $3 \times$ $10^{9}$ of ${ }^{252} \mathrm{Cf}$ fission neutrons per square centimeter. We observed decreases in resistivity of at most 10 percent. This, together with the extreme sensitivity of the method to moisture led to the conclusion, in contradiction to a published report, that the resistivity of cellulose acetate would not make a useful neutron dosimeter. This work has been accepted for publication. 


\title{
J. M. Cassel
}

\author{
Task Leader
}

The objective of this research is to provide test methods and standards to sectors of the health care industry that make critical use of materials. Materials research and techniques are pursued with the goal of developing dental adhesive composite restorative materials of greater durability and wear resistance, improved base metal alloy alternatives to the costly gold alloy dental prostheses, and test methodology applicable to porcelain/alloy combinations. Knowledge of the structure and physical chemistry of biologically important calcium phosphates is used to develop improved preventive dental techniques. Improved test methodology is sought for characterization of the surfaces of surgical implant materials for defining the interactions that occur thereon. Means for characterizing the porosity of low modulus, polymeric, medical implant materials are being investigated.

Dental research directions in support of the objective are established collaboratively with the American Dental Association, the National Institute of Dental Research, and the Department of Defense Medical R\&D Command. Medical materials research is guided by close interaction with the Bureau of Medical Devices, Food and Drug Administration, and the ASTM F-4 Committee (Medical and Surgical Materials and Devices.)

\section{Dental Composite Chemistry and Development}

G. M. Brauer, J. M. Antonucci, J. W. Stansbury, J. M. Cassel, S. Venz ${ }^{1}$, and R. L. Bowen ${ }^{2}$

With the view that reducing polymerization shrinkage and residual unsaturation in dental resin systems would make them more useful in efforts to achieve adhesive bonding, better color stability, and improved durability in composite restorative systems, we have continued to investigate new comonomer combinations whose structures would suggest their applicability for this purpose. A particularly promising monomer is the high molecular weight, moderately viscous polythiol pentaerythritol tetra (3-mercaptophopropionate) (PETMP). It has potential not only as a useful comonomer but also as a polymerization accelerator. With multifunctional methacrylates that readily undergo free radical self-addition reactions of vinyl groups to create a propagating polymeric chain, the use of polythiols leads to a hybrid polymer composed of polymethacrylate and polythioether elements in the network structure. Preliminary results using infrared spectroscopy indicate there is a significant reduction in vinyl unsaturation when modest concentrations ( $<5$ percent by weight) of PETMP are employed in a typical dental resin formulation. Levels of PETMP between 1 and 5 percent have produced composite materials with excellent esthetics and color stability. The improvement in color stability and esthetics in many of the resin systems examined may be due to the fact that use of PETMP allows a reduction in the concentration of amine accelerator, a recognized source of color problems with composite restorations. A further important potential advantage to be explored with PETMP is that it appears to enhance the storage stability in the dissolved state of such highly biocompatible polymerization promotors as ascorbic acid.

We have found the bulky monomethacrylate, isobornyl methacrylate (IBM) can be used to dilute 2,2-biis[P-(2-hydroxy-3-methacryloxy-propoxy)phenylene]-propane (BIS-GMA) and composites with good setting characteristics, color and mechanical properties are obtained. Unlike many long chain alkyl methacrylates that have sluggish ambient temperature rates of polymerization, IBM is more reactive even than methyl methacrylate. Homopolymers of IBM in contrast to other alkyl methacrylates have a slightly higher glass transition temperature $\left(T_{g}\right)$ than that for poly(methyl methacrylate) (PMMA) and this may prove advantageous in developing greater durability in composite restorations. However, when used as a comonomer with a hydrophobic, highly fluorinated polymethacrylate for purposes of reducing water sorption, the IBM appears to yield only marginal strength composites.

Our previous investigation of the silanization treatment of composite filler particles had shown that incorporating certain n-alkyl amines and selecting a hydrocarbon carrier for 3-methacryloxypropyltrimethoxysilane increased the reinforcing capability of the filler particles. This beneficial effect has now been shown to be optimized using a 2 percent solution of $n$-propyl amine and 0.5 percent of silanizing agent in cyclohexane. Initial tensile strengths of composites is improved significantly and the rate of water-induced loss of strength as determined for two and six months at $37{ }^{\circ} \mathrm{C}$ is reduced.

\footnotetext{
${ }^{1}$ Guest Worker, Free University of Berlin

${ }^{2}$ Research Associate, American Dental Association
} 
G. M. Brauer, R. L. Bowen², J. M. Antonucci, J. W. Stansbury, D. W. Misra ${ }^{1}$, E. Cobb $^{3}$, and J. M. Cassel

While effective bonding to tooth enamel is presently achieved by means of acid etch treatments, similar treatments of dentin have not shown satisfactory results. We are currently investigating several potential means of resolving this problem.

Alkylcyanoacrylates polymerize rapidly at room temperature and certain ones have been previously shown to yield a very strong initial bond with dentin that unfortunately is weakened significantly on exposure to water. To address this shortcoming we have begun an effort to prepare a cyanoacrylate with cross-linking capability, i.e., allyl 2-cyanoacrylate. The synthesis involved esterification of cyanoacetic acid to allyl cyanoacetate which was then reacted with paraformaldehyde to give poly (allyl 2-cyanoacrylate). While we have made repeated efforts to depolymerize this polymer to yield the desired monomer, we have not been successful to date and currently we are examining alternative means for its preparation.

A new bonding procedure that uses an acid mordanting solution, a surface active monomer, and a coupling agent to prepare dentin and enamel for bonding to composite resins appears to have much promise. An aqueous solution of ferric oxalate is applied, washed with water, and blown dry. An acetone solution of the surface active comonomer NTG-GMA, the adduct of N(p-tolyl)glycine and glycidyl methacrylate is applied, followed by pure acetone and drying with air. An acetone solution of the addition reaction product of pyromellitic dianhydride and 2-hydroxyethylmethacrylate is then applied and the surface blown dry before pressing a composite resin formulation against the surface and allowing it to harden (polymerize). Scanning electron microscopy results show that the iron oxalate solution alters the tooth surface layers. The coupling agents then provide molecules which are bound to the modified surface and can polymerize with the resin of the composite material applied subsequently. Bond strength measurements confirm that the strong bonding initially achieved is retained on exposure to water for up to five days. A real possibility exists for improving treatment of erosion, root caries, and other conditions by reducing the amount of dentin that must be cut for mechanical retention.

Dental cements with good biocompatibility, improved mechanical properties and resistance to disintegration on exposure to the oral environment are needed. Zinc oxide-eugenol dental cements, while possessing many desirable properties, are relatively soluble in oral fluids and adhere poorly to acrylic restorations, bone, or dental tissues. Furthermore, because of the electron-rich phenolic hydroxyl group in eugenol, these cements inhibit free radical polymerization and hence hardening in any dental resin or composite system subsequently placed in contact. Previous research to develop substitutes for the eugenol component has not produced cements with adequate physical properties. Recently, however, we have developed a non-eugenol based cement that combines zinc oxide, ethoxybenzoic acid (EBA) and esters of vanillic acid. It has been demonstrated to have not only desired physical properties but certain important chemical properties. These cements do not inhibit free radical polymerization, and adhere strongly, even in water, to nonprecious metals, amalgams and composites. They do not disintegrate in water and considerably exceed the requirements of the ANSI/ADA Specification No. 30. Thus, a portion of the cement, when used as a temporary filling, can be retained as a base for acrylic resin restorations. The material, with suitable modifications, looks most promising as a base, temporary restorative, and endodontic cement. Cementitious restoratives with drastically improved mechanical properties which could find applications as long-lasting temporary fillings were prepared by incorporating a dimethacrylate monomer, peroxide initiator, amine accelerator, and glass reinforcing fillers into the cement formulations. We have prepared (by synthesis) sufficient quantities of a promising vanillate, a hexyl vanillate, to allow biocompatibility testing. Samples of the powder and liquid have been sent to six institutions for biological evaluation of the basic formulation.

To increase the strength of these cements beyond the already respectable levels achieved so that increased dental application can be made, we have investigated cements prepared with $\mathrm{ZnO}$, $\mathrm{Al}_{2} \mathrm{O}_{3}$, hydrogenated rosin and silanized glass (coated with benzoyl peroxide) as the powder and hexyl vanillate, decamethylene dimethacrylate, a fast acting amine accelerator and a stabilizer as the liquid. Cured cements with good working properties and strengths, four to seven times that obtainable with zinc oxide-eugenol formulations, were obtained. These vaniliate-based cement modifications offer promise as intermediate restorative materials. 


\title{
Wear Mechanisms, Wear Testing and Improving Durability in Dental Composite Materials
}

W. Wu, J. E. McKinney, E. E. Toth, and J. M. Cassel

Development of improved dental restoratives requires proper evaluation criteria to predict the long term in vivo performance of these materials. Microdefect analyses involving a photographic silver staining procedure and a small angle x-ray scattering (SAXS) technique have been applied to quantify the extent of subsurface damage in both in vivo and in vitro worn composite materials. Since we find that there is subsurface damage beneath the nonstress bearing as well as the stress bearing surfaces in clinically worn restorations, we have theorized that the oral environment plays an important role in the deterioration process. Consequently, the diffusion of food simulating chemical solvents into dental composites and the influence of this diffusion in softening the polymeric matrix as a prime step toward loss of material has been investigated.

Initial measurements of diffusion coefficients for water in typical composites, using a quartz spring mass sorption technique, indicated that the diffusion rates were Fickian and that both diffusion rate and water sorption were significantiy reduced in composites cured at 60 vs $37^{\circ} \mathrm{C}$. By infrared measurement of residual double bond concentration in the dental resin as a function of the curing time and correlation of these results with differential scanning calorimetry measurements of the curing that occurs as the temperature is raised from the initial $37{ }^{\circ} \mathrm{C}$, we have shown that the more delayed the temperature rise is, the less is the effect on curing. This would dictate that if increased curing by heating were to be made clinically viable, it would need to be accomplished almost immediately following placement of the restoration.

Measurements of Knoop hardness on a conventional, a strontium glass filled, and a visible light activated composite after each was cured at $37{ }^{\circ} \mathrm{C}$ and subjected to immersion in food simulating solvents for one week demonstrated that each was softened to the greatest extent by 75 percent ethanol/water concentrations. The largest effects at this ethanol concentration were observed with the conventional composite and the least with the light activated material. Immediately following completion of the immersion in the food simulating solvents, wear measurements were made using pin on disc wear equipment. While the wear analysis is not completed, the wear for both the conventional and the light activated composites when plotted against the solubility parameter of the food simulating medium follow the same trend, e.g., maximum wear rates at 75 percent ethanol concentration, as observed with the Knoop hardness measurements. When the in vitro wear rates of composites cured at 25 and $60{ }^{\circ} \mathrm{C}$ and then immersed in 75 percent ethanol in water media were compared, the wear rates of the composites cured at the higher temperature were one-fourth that of the lower temperature-cured material. These results are in perfect accord with the preliminary diffusion data referred to earlier and support our contention that ways (heat or otherwise) must be found to significantly increase the degree of polymerization in the composite resin during the restoration process.

\section{Composite Restorative Materials}

\author{
J. M. Antonucci, G. M. Brauer, S. Venz, N. W. Rupp, and J. M. Cassel
}

Restorative dentistry is that form of dental practice that utilizes the most time of the Army dentist as well as the soldier dental patient. Composite materials are prime components in restoration procedures. Greater durability and satisfaction would be achieved with such materials if the marginal adaptation (resistance to microleakage) were improved. For the Army it is also important to improve the storage stability of such materials and earlier efforts in this project have defined the prime sources of storage instability and practical means for optimizing storage stability with presently available materials.

This year we have demonstrated the improved sensitivity in microleakage measurement that can be achieved by using a silver staining technique as an alternative to a more cumbersome radiotracer x-ray film procedures. Microleakage determinations have been made on a series of experimental composites formulated to be hydrophobic rather than mildly hydrophilic as are current commercial dental composite materials. While a dual silanization procedure for fluorinating as well as silanizing glass filler particles did not reduce water sorption in the composite, the increased compatibility of such glass with fluorinated monomers may offer a means of achieving higher filler loadings and hence improved physical properties if needed. Combining a commercially available fluorinated diluent comonomer, pentadecafluoroctyl methacrylate, with two commercially available nonhydroxylated analogs of BIS-GMA, the basic resin in conventional dental composites, resulted in a composite with adequate physical properties but did not enable us to reduce water sorption significantly.

Since we hypothesize that to significantly lower both microleakage and water sorption we need a resin system that is hydrophobic and that on curing shrinks minimally, we have begun examination of composite formulations derived with the very large experimental prepolymer, PFMA, a highly fluorinated polymeric (approximately 10 repeating units) comonomer with multiple methacrylate groups for chain extension and cross-linking. To achieve workable viscosities we are diluting with highly hydrophobic comonomers such as 1,10-decamethylene dimethacrylate or neopentyl glycol dimethacrylate. Results to date indicate promise in terms of reduced water sorption and microleakage and respectable physical properties. 
J. A. Tesk, R. W. Hinman ${ }^{4}$, W. deRijk², D. L. Menis ${ }^{2}$, G. Wideras, J. Sanecki ${ }^{6}, 0$. Franklin ${ }^{7}$, and J. Arnold ${ }^{8}$

A major cause of failure in porcelain-fused-to-metal restorations involves cracking or delaminating of the porcelain veneer. Apropos to this situation we developed a new concept for evaluation of porcelain-to-metal bond strength. The test uses four-point bending of a metal strip to which porcelain squares have been fused and measures tensile strengths of porcelain-to-metal bonds (without influence from shear stresses). Recent finite element model (FEM) calculations confirm that simple beam theory provides an adequate description of the stress within the system. The effective stress is virtually the same as the normal tensile stress (i.e., other stresses, such as shear stresses are sma11) and both are essentially the same (to within about 6 percent on the average) as stresses calculated by beam theory. Moreover, a smooth transition of stress from metal through porcelain is indicated by FEM calculations which were conducted using an elastic modulus for metal, $E_{m}$, twice that for porcelain, $E_{p}$, i.e., $E_{m} / E_{p}=2$.

Preliminary test results have shown that two to four strength determinations can be made from one test specimen. Application of St. Venant's principle resulted in specimen test design intended to leave such successive strength determination independent of previous fractures. What is determined is either the porcelain-to-metal bond strength or the porcelain fracture strength. Test results show that for strong porcelain-to-metal bonds only porcelain fractures are recorded. For weak bonds at interfaces only interface fractures are recorded. In other cases, mixed failures occur, i.e., a porcelain-to-metal bond failure may be preceded by a porcelain fracture and followed by two more porcelain fractures; other possibilities are obvious. Because the order of failures (fractures) is expected to be toward continuously increasing strength values, statistical interpretation in terms of ordered statistics will be investigated. Experiments are currently in progress to develop meaningful statistical data as the preliminary testing has been essentially completed.

Industry has expressed great interest in the new test methodology as it offers the potential of producing quantitative as well as qualitative information on porcelain-to-metal bonding and on relatively easily prepared specimens which yield several pieces of information per test run. Three dental companies committed R\&D personnel to work as visitors for one week in an evaluation of the test methodology.

We have previously developed rationale for utilizing the change in gap of a porcelain veneered split metal ring as a measure of porcelain/alloy stress compatibility. Recent work has centered on development of the FEM which incorporates the viscoelastic properties of porcelain. Difficulties have been experienced with the continuity of the computer program and these are currently being worked on. This, coupled with a recent concentration of effort on the porcelain-fused-to-metal bond, testing and castability evaluations has resulted in delaying progress. This work is expected to renew in intensity as we are joined by guest workers from Germany and Japan.

The thermal expansion behavior of many experimental porcelain compositions have been determined. These porcelains have been formulated to achieve very low fusing temperatures, i.e., below $800{ }^{\circ} \mathrm{C}$. The coefficient of thermal expansion usually varies with temperature and, in general, the relationships can be grouped into three different classes: (1) a monotonic increase with temperature; (2) an irregular change with temperature; and (3) no change with temperature. These findings demonstrate that matching the coefficients of thermal expansion of metal and porcelain at only one high temperature as is usually done may not lead to an optimal porcelain-to-metal system.

Mathematical analysis has been performed on the data obtained in studies of the effects of various ingredients that influence the fusion temperature in experimental porcelains. It was found that a wide range of $\mathrm{B}_{2} \mathrm{O}_{3}$ concentrations has a surprisingly small effect on the fusion temperature of these experimental porcelains.

During the past year new investments for use with titanium metal have been investigated. The preliminary studies showed that $\mathrm{SiO}_{2}, \mathrm{Al}_{2} \mathrm{O}_{3}$ and $\mathrm{AlPO}_{4}$ are ingredients to be avoided. Molten titanium acts as an extremely strong reducing agent and reduces $\mathrm{SiO}_{2}$ while forming a titanium oxide. It was found that similar reactions occur with aluminum oxide and aluminum phosphate. Three oxides that are

\footnotetext{
${ }^{4}$ Research Associate, U. S. Navy

SUniversity of Illinois

6Visitor, Howmedica, Inc.

${ }^{7}$ Visitor, Dentsply International

${ }^{8}$ Visitor, Williams Gold
} 
relatively stable have been identified, namely those of magnesium, yttrium and zirconium. However, moldcoats of these oxides, used as liners with conventional phosphate-bonded investments, were unsuccessful because of poor adherence to the investment.

\title{
Dental Casting Alloys and Investments
}

\author{
J. A. Tesk, R. M. Waterstrat ${ }^{1}$, R. W. Hinman ${ }^{4}$ W. deRijk ${ }^{2}$, and D. L. Menis ${ }^{2}$
}

Dental casting alloys based on gold or cobalt have, in recent years, faced serious problems of cost and availability. Though these problems have eased somewhat, they could recur at any time. The use of nickel alloys as substitutes has faced some concern for their toxicity and induced allergic responses in certain patients. Titanium-based alloys would seem to offer a satisfactory alternative, being strong, corrosion-resistant, relatively inexpensive, widely available, and nontoxic. A study of the effects of interstitial solutes $(O, N, C)$ on phase equilibria in Ti-rich Ti-Al alloys was initiated. Also, high-temperature neutron diffraction experiments are planned in collaboration with the NBS reactor group. Equipment designed to permit observations of alloys to $1200{ }^{\circ} \mathrm{C}$ is under construction.

With the introduction of numerous dental alloys as alternatives to gold alloys, it becomes increasingly important to develop a castability test that is indicative of an alloy's ability to fill a mold of specific design under a set of prescribed conditions. Such a test has been under development in this laboratory and in its present stage assesses the percentage, $C_{v}$, of cast segments of a polyester grid pattern, figure 1. We have applied the test to a number of dental alloys using the manufacturer's recommended mold and alloy casting temperatures. Results of $C_{v}$ determinations are shown for several dental alloys as they are cast using different investments, figure 2.

The following observations support the validity of applying the test method: (1) some of the presently employed alternative alloys compare favorably in castability to those of high noble content; (2) the brand of mold investment used can produce a significant effect on the castability of some alloys; (3) as expected, the castability value increases as mold and casting temperatures increase; (4) an optimal mold and casting temperature can be discerned for each alloy beyond which castability values show little change; (5) for each alloy a different amount of heat above liquidus ("superheat") is required to produce the optimal casting temperature; (6) the effect of mold temperature on castability may be greater than previously thought for some alloys and less significant for others; and (7) as the limiting value for castability is approached $\left(C_{v}=100\right.$ percent) the standard deviation (SD) from the mean determination of $C_{v}$ appears to decrease. Hence, larger SDs for some alloys may well be indicative of potential for further optimization of conditions for casting of that alloy.

As can be seen from the foregoing observations, the investment, the temperatures used, and probably many other factors have to be taken into account when comparing the castability of dental alloys. As a further example of this, preliminary studies using torch casting indicate that the castability value for some alloys is altered significantly from those cast by induction melting. A question that naturally arises is: what threshold castability value is acceptable? This again brings up the point previously made, that the test method should be used where relative comparisons are desired and that the significance of the absolute castability value of any alloy system by itself is an unanswered question and is at present a matter of conjecture.

It is apparent from the data that the standard deviation of the castability value, $\mathrm{SD}_{\mathrm{CV}}$, is a function of $C_{v}$, the casting temperature, $T_{c}$, and mold temperature, Tm, i.e., $S D_{C v}=f\left(C_{v}, T_{c}, T m\right)$. The form of the relationship has not yet been determined (standard statistical transformations have been found not to apply and are the subject of continuing work). We only wish to note at this point that if a universally applicable relationship can be found it will significantly reduce the number of experiments required to completely determine castability for any alloy as a function of temperature. The benefits of such a relationship should be obvious to those engaged in quality control, product development or setting of standards.

A final point should be made on the repeatability of the results as reported here. One base metal alloy having a castability value toward the center of the range was cast on five separate occasions to monitor this aspect. The results were statistically indistinguishable.

\section{Dental Chemistry and Preventive Dental Treatments}

W. E. Brown ${ }^{2}$, L. C. Chow ${ }^{2}$, M. Mathew ${ }^{2}$, M. S. Tung ${ }^{2}$, G. L. Voge ${ }^{2}$, S. Takagi ${ }^{2}$, T. M. Gregory ${ }^{2}$, and A. Uchida'

The objective of this project is to develop and apply fundamental information on the solubilities and crystallographic structures of biologically important calcium-containing phosphate compounds to the 


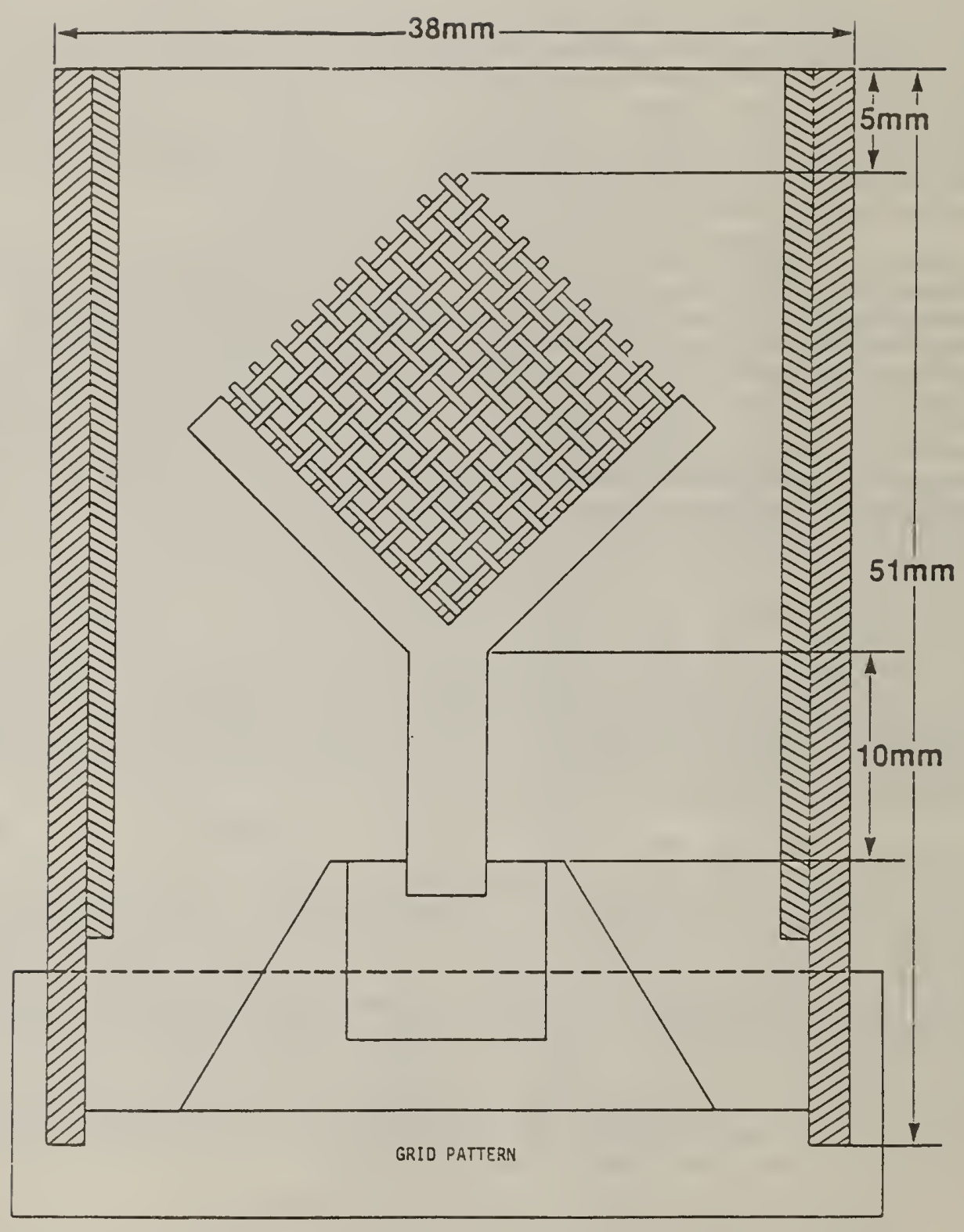

Figure 1. Grid pattern. 


\section{CASTABILITY}

\section{USING STANDARD EVALUATION PROCEDURE WITH DIFFERENT INVESTMENTS}

Alloy $\mathbf{N}$

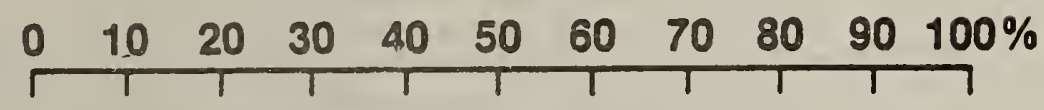

Investment

A

B

C

D

E

S.D. $\longrightarrow$

Alloy $\mathbf{P}$

Investment

A
B
C
D
E
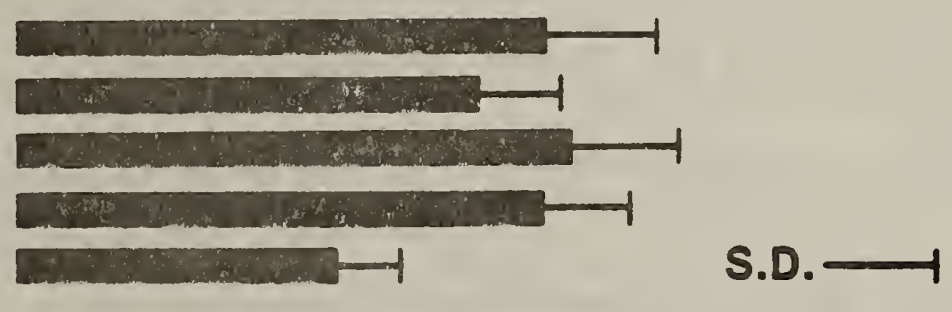
development of improved dental caries preventive techniques. Important portions of this effort are concerned with (1) development of physico-chemical models of the caries process to include insight into the mechanisms by which impurities and defects are incorporated onto tooth enamel, and (2) application of solubility phase diagram analysis and kinetic data to the development of more effective enamel acid-etch bonding treatments and improved topical fluoride procedures.

During the past year, the crystal structures of $\mathrm{CaK}_{3} \mathrm{H}\left(\mathrm{PO}_{4}\right)_{2}$ and $\mathrm{Ca}\left(\mathrm{NH}_{4}\right) \mathrm{PO}_{4} \cdot 7 \mathrm{H}_{2} \mathrm{O}$ were determined. Structure analysis of a calcium phosphate-urea complex, predicted to possess a layer-type structure, has revealed it to be a non-layer type compound. A structural study of natural and synthetic crystals of bobierrite, $\mathrm{Mg}_{3}\left(\mathrm{PO}_{4}\right)_{2} \cdot 2 \mathrm{H}_{2} \mathrm{O}$ revealed the positions of the hydrogen ions in this member of an important and varied class of compounds. Research on the structure of octacalcium phosphate, $\mathrm{Ca}_{8} \mathrm{H}_{2}\left(\mathrm{PO}_{4}\right)_{6} \cdot 5 \mathrm{H}_{2} \mathrm{O}$, and its role in biomineralization continues to be a major activity. During the report period, the refinement of the crystal structure was completed; important evidence for its participation in the formation of enamel mineral was gained. A chemical procedure was developed that for the first time makes it possible to measure separately the rate of its formation and rate of its conversion to the basic mineral of bone and tooth.

The work on caries mechanisms is carried out on three levels: (1) theoretical considerations to develop physicochemical principles which might govern the caries process; (2) bench-scale experiments to validate such principles; and (3) ultramicro studies on tooth sections to establish on a quantitative basis the events occurring within the tooth itself.

Theoretical considerations focus on the idea, heretofore largely ignored, that the relative rates of diffusion of ions into and out of the lesion are important parameters in the caries process. It was predicted and subsequently confirmed that factors such as the presence of fluoride, the membrane-like behavior of the outer surface of the tooth and the plaque, and neutral salts normally present in the saliva are among the important parameters affecting the rate of the caries process. Bench-scale studies showed that the rate of demineralization was increased by a factor of 80 when the membrane transmitted positive ions selectively as compared to when it transmitted negative ions selectively. Also, $\mathrm{F}^{-}$greatly reduced the rate of demineralization (this effect appeared to be greatest at pHs below about four). These results provide further evidence in support of a cariostatic mechanism for $F$ developed at NBS and suggest that the caries resistance of teeth may be increased by modifying the selectivity of the tooth surface to transmission of ions. In ultramicro studies, good progress was made in developing pieces of equipment and procedures for working in the nanoliter (10-9 1 iter) sample range. The techniques have been extended to include analysis of plaque samples where the volumes of plaque are sufficiently small so that they can be collected from a single tooth.

In addition to the studies on the role of fluoride in the caries mechanism, work continues on the new topical fluoridation procedures developed in these laboratories. These procedures are based on the idea that tooth mineral (primarily $\mathrm{Ca}_{5}\left(\mathrm{PO}_{4}\right)_{3} \mathrm{OH}$ ) reacts very slowly with $\mathrm{F}^{-}$. However, as a part of the fluoridation treatment, some of the $\mathrm{Ca}_{5}\left(\mathrm{PO}_{4}\right)_{3} \mathrm{OH}$ can be converted to another compound, $\mathrm{CaHPO}{ }_{4} \cdot 2 \mathrm{H}_{2} \mathrm{O}$, which reacts rapidly with $F^{-}$. Through the use of procedures based on this principle, it has been found possible to greatly increase the permanently-held $F^{-}$content of teeth. The clinical relevance of this increased $\mathrm{F}^{-}$content remains to be established, but it is plausible that it should be beneficial.

A successful remineralization procedure would drastically alter the procedures used to treat incipient lesions. During this period, initial steps were taken to develop procedures for testing remineralization formulations based on prior basic studies carried out in these laboratories. The initial results are encouraging. Substantial progress was made in developing techniques to remineralize exposed and sensitive root surfaces. A procedure was developed which by laboratory criteria appears to be a successful remineralization procedure. It is expected that preliminary clinical evaluations will be carried out in the near future.

\section{Clinical \& Laboratory Investigation of Dental Amalgams, Composites \& Cements}

\section{N. W. Rupp ${ }^{2}$, G. C. Paffenbarger ${ }^{2}$, and R. M. Waterstrat ${ }^{2}$}

Dental restorations vary considerably with time. Among the factors influencing the success or failure are: (1) the material and how it was handled by the operator; (2) the cavity preparation; and (3) the patient's home care. Sorting out the factors contributing to the durability of a restoration requires a careful combination of clinical studies and laboratory analyses.

Observations of amalgam restorations placed 7 to 8 years ago are continuing in the clinic and laboratory. Amalgams that are high in copper content and are highly creep-resistant continue to be superior. For creep below 2 percent marginal integrity is more dependent on operator manipulation than on creep. For example, creep values of 0.2 or 0.8 percent do not seem to correlate with the quality of the margins. Alloy composition and the resultant amalgam composition influence the aging characteristics. Laboratory measurements of dimentional change have been made for six years on specimens stored at 23,37 , and $60^{\circ} \mathrm{C}$ on either unrestricted specimens or in specimens restricted by placement in simulated Class $V$ Cavities in steel blocks. Zinc, tin, and mercury contents, each independently, 
affect the dimensional changes: increasing the zinc and mercury contents seems to cause an expansion, while tin over 28 percent usually results in shrinkage. There are, however, some exceptions. As we gain a better understanding of the rheology and the internal phase changes, it should be possible to extend the useful life of amalgam restorations.

One of the principal defects in composite restorations continues to be imperfect sealing of gingival margins. Laboratory studies confirmed that microstructural bonding of composites to enamel successfully prevents microleakage, and that the paucity of enamel at the gingival margin obviates sealing through the use of acid-etch bonding. Currently the only commercially available materials that are adhesive to dentin are those with carboxyl groups. Clinical observations were made with glass ionomer cements which contain these groups, and the adhesion does appear to be good; the visual opacity of these materials continues to be a problem.

There is also an available product that utilizes a coupling agent that is polymeric and contains carboxyl groups. This system does not call for treatment of the dentin with citric acid (which opens the tubules to irritants). In vitro and in vivo testing of this coupling agent-composite system is being pursued to assure that this combination can be advocated for general use. If so, this will reduce staining and decay at the gingival margins of composite restorations.

\section{Adhesion of Synthetic Polymers on Solid Surfaces}

\section{R. E. Dehl and W. H. Grant}

The adhesion of polymeric protective coatings, with its many important commercial applications, is hampered by a lack of fundamental understanding of the interaction of polymer molecules with solid surfaces. It is the energy of adsorption of individual polymers with a surface which determines the adhesive strength of an adhering polymer film, and there is a need for more specific information about such factors as the role of individual polymer segments attached in governing the adhesive strength. One measure of the energy of attachment is the rate at which polymers desorb from solid substrates when the coated surface is placed in contact with a suitable solvent. The results of previous NBS studies, using radiolabeled polystyrene to detect the amount of polymer remaining on the surface, indicate that the rate of desorption decreases with increasing molecular weight of the polymer and with the amount of polymer initially adsorbed. We have also previously reported that the rate of desorption of tritium-labeled polystyrene from solid surfaces depends not only upon the amount adsorbed, but also upon the way in which a given level of adsorbance is achieved. Such factors as the time of adsorption, which can be controlled by adjusting solution concentration, and preadsorption to higher adsorbance levels were found to affect the desorption rate from the same adsorbance level. A model was proposed to explain these phenomena, based upon two or more types of adsorption sites having different energies of attraction for polymer molecules.

During the past year, we have found that the rate of adsorption and desorption studied over long periods of time may be complicated by the tendency of high molecular weight (MW) species to degrade in time. One polystyrene solution which had been used for several months was found by gel permeation chromatography (GPC) to have a broader MW distribution and a lower average MW than a fresh solution of the polymer. Whether this degradation is due to chemical attack or the shearing action of the reciprocating stirrer bath used in these studies is not known at present. However, it has also been found that the GPC elution curves of fresh polymer solutions do not differ significantly from those obtained after shaking the solutions for six weeks in the stirrer bath, indicating minimal degradation over this time interval. Future studies will be conducted within a six-week period in order to minimize the effects of polymer degradation.

\section{Characterization of Porosity of Porous Implant Materials}

\section{R. E. Dehl}

Porous synthetic biomaterials are currently being used as implants, where they become stabilized by the ingrowth of hard or soft natural tissues. Both the rate and the type of tissue ingrowth are affected by the porosity of the materials, and it is important to be able to characterize the porosity by reliable and reproducible methods. During the first phase of this work, which is being carried out in cooperation with the Bureau of Medical Devices, Food and Drug Administration, we have surveyed the published literature pertaining to porous polymeric implant materials and initiated studies of pore size and pore volume using mercury intrusion porosimetry. The materials currently being investigated are porous polyethylene (PPE) and a porous composite of polytetrafluoroethylene and pyrolitic carbon.

Our initial studies indicated qualitatively that, contrary to some expressed fears, mercury intrusion experiments apparently can be performed on the relatively soft composite material without distorting the pore structure by mechanical compression. During the past year we have quantified these observations by performing compressive stress-strain measurements on both materials. The composite has highly anisotropic mechanical properties due to its laminated construction, the compression modulus being about five times greater in the direction parallel to the laminations, as compared with perpendicular compression. 
The pore volume in these materials has been measured by three different methods and the pore "size" by two methods, namely mercury intrusion porosimetry and an indirect method based upon the specific surface area and specific pore volume. The surface areas have been measured both by the Brunauer, Emmet, Teller (BET) inert gas adsorption method and by the mercury porosimetry method in which the surface area is proportional to the integrated area of a pressure-volume mercury intrusion curve. Both methods agree that the PPE has a smaller surface area than the composite, but the BET method indicates a much greater difference, for reasons which are currently under investigation.

The accuracy of the mercury intrusion method for measuring pore size has been tested by performing an estimated random error analysis of the experiment and plotting volume-weighted pore size distribution curves based upon these error limits. The resulting curves show that the experimental precision is not sufficient to produce well resolved distribution curves, over much of the pressure range pertinent to these materials, but that the precision increases rapidly with increasing pressure, corresponding to smaller pores.

Quantitative studies of porosity are also being initiated, using the methods of quantitative stereology. The accomplishment of this project's objectives will be of significance to the medical device industry and medical implant standards development, as well as to the users of porous implant materials.

\section{Surgical Bone Cement Modification}

\section{J. A. Tesk, G. M. Brauer, W. Wu, and J. E. McKinney}

While surgical bone cement is a major asset in orthopaedic practice, it has deficiencies that are of much concern, particularly in total joint replacement. Failure at the cement/bone or cement/implant interface is considered to be an important step in reducing the life time of such total joint replacements as those used to reconstruct knee and hip joints.

With the view that completely new bone cements are not likely to be forthcoming, we have directed our efforts to improving the presently available materials and techniques. A first effort has been to examine the potential of some less toxic new amines, proven in our lab to be more reactive with the dental resin, BIS-GMA, than amines currently employed. We have found that dialkylaminophenethanol used with benzoyl peroxide as initiator will give faster curing of bone cement. This cure time is reduced even more by addition of equimolar concentrations of benzoic acid. We have initiated efforts to reduce the heat liberated on polymerization by bone cement by investigating the substitution of the reactive but higher molecular weight dicyclopentyloxyethyl methacrylate for presently used methyl methacrylate.

Based on the results of the amine studies additional tests will be chosen from among those considered in the original protocol. One such avenue of investigation involves determining if performance may be expected to be improved by precoating of a prosthetic stem with highly cured polymer. The properties of the polymer are expected to be improved relative to properties at the interface of a stem and self-curing acrylic; the stem will be a heat sink and the lower interface temperature should inhibit obtaining an optimum cure. A precoated stem, however, will be insulated from the self-curing acrylic, the polymer itself should be solvated somewhat by the self curing monomer, and interface properties improved by the post curing of the precoat.

At present, 10 cobalt-chromium rods 3/8" diameter have been cast (courtesy, Howmedica, Inc.) and prepared for testing with polymer. Initial tests are in progress regarding comparative pushout force-displacement evaluation of coated and uncoated rods.

\section{Dental and Medical Materials Standards}

J. M. Cassel, J. A. Tesk, G. M. Brauer, and N. W. Rupp ${ }^{2}$

Dental and medical implant standards development is strongly supported by persons in positions of Committee, Subcommittee, and Task Force Chair roles in ANSI MD 156, Dental Materials, Instruments, and Equipment, and in ASTM F-4, Medical and Surgical Materials and Devices. Additional leadership roles are fulfilled through the chairpersons role in USA TAG ISO/TC 150, Implants for Surgery, and USA TAG ISO/TC106, Dental Materials, Instruments, and Equipment, and through participation in the Executive Committee of the ANSI Medical Device, Standards Management Board.

Considerable effort has gone into drafting four new or revised ANSI/ADA dental specifications and comparable ISO standards. These cover alloys, mercury and devices used in the preparation of amalgams.

An active role was taken in the planning for and organization of U. S. participation in the Fifth Meeting of ISO/TC 150, Implants for Surgery, held in Washington, DC. 


\section{Invited Talks}

Standardization of Gas Transmission Measurements

Edgewood Arsenal, Edgewood, MD

J. D. Barnes

January 20, 1982

A Computer-Controlled Gas Transmission Measuring Apparatus

40th Annual Technical Conference, San Francisco, CA

J. D. Barnes

May 10, 1982

Standard Reference Materials for Gas Transmission Measurements

18th State of the Art Symposium, American Chemical Society, Washington, DC

J. D. Barnes

June 14, 1982

Bonding at the Polymer/Tooth Surface

6 th Annual Meeting of the Society for Biomaterials, Orlando, FL

R. L. Bowen

Apri 1 24, 1982

Bonding to Dentin--At Last a Reality

American Society for Dental Aesthetics, New York, NY

R. L. Bowen

May 5, 1982

Electric-Field Induced Changes in the Phase \& Orientation of Polyvinylidene Fluoride Crystals IUPAC Macro ' 82 Conf., Amherst, MA

M. G. Broadhurst

July 16,1982

Piezo- and Pyroelectricity in Polymers

The Materials Research Society, Boston, MA

M. G. Broadhurst

November 16, 1981

Piezoelectric Polymers as Potential Soil Stress Gages

Air Force Weapons Laboratory, Albuquerque, NM

M. G. Broadhurst

January 6, 1982

Overview of Piezoelectricity, Pyroelectricity \& Ferroelectricity in Polymers

Workshop on Polymers as Synthetic Metals, Los Alamos, NM

M. G. Broadhurst

Apri1 26, 1982

Gruneisen Equation of State for Polymers

Symposium at Los Alamos National Laboratory, Los Alamos, NM

M. G. Broadhurst

Apri 1 29, 1982

Initiator-Accelerator Systems for Dental Applications

ACS Symposium, Las Vegas, NV

G. M. Brauer

Apri 1 1, 1982

Crystallography and Thermodynamics of Calcium Phosphates Related to Biomaterials Martin Marietta Laboratories, Baltimore, MD

W. E. Brown

January 19, 1982

Thermodynamics of Hydroxyapatite Surfaces, 6th Intl. Summer Conference on Chemistry of Solid/Liquid Interfaces, Dubrovnik, Yugoslavia

W. E. Brown

June 25, 1982 
Fabrication and Characterization of Ultra-Drawn Thick PVDF Transducers

IUPAC Macro ' 82 Conference, Amherst, MA

A. J. Bur

July 16,1982

A Dental and Medical Materials Program at NBS

Johns Hopkins University, Baltimore, MD

J. M. Cassel

April 13, 1982

Calorimetric Studies \& Spontaneous Thermal Effects in Poly(chlorotrifluoroethylene)

lith North American Thermal Analysis Society Conference, New Orleans, LA

S. S. Chang

October 21, 1981

Topical Fluoridation \& Physico-Chemical Mechanisms of Fluoride Cariostasis

University of Alabama Dental School, Birmingham, AL

L. C. Chow

November 19, 1981

Crystal Structure of Polytetrafluoroethylene as a Function of Temperature \& Pressure

IUPAC Macro ' 82 Conference, Amherst, MA

E. S. Clark

July 13,1982

Crystal Structure of the Low Temperature Form (Phase II) of Polytetrafluoroethylene American Crystallographic Assn. Spring Meeting, National Bureau of Standards, Washington, DC

E. S. Clark

March 29, 1982

A New Test Method for Determining the Environmental Stress-Crack Resistance of Ethylene Based Plastics ASTM Subcommittee D20.12.03, Toronto, Canada

J. M. Crissman

June 23, 1982

Field Induced Phase Transitions \& Piezoelectricity in Vinylidene Fluoride Polymers and Copolymers IUPAC Macro ' 82 Conference, Amherst, MA

G. T. Davis

July 16,1982

Polarization Distribution in PVF 2 as Measured by the Thermal Pulse Method and Recent Results on the Piezoelectric Retention Characteristics of PVF ${ }_{2}$ Upon Heating to Melting Thomas-CSF

Laboratorie Central de Recherches, Orsay, France

A. S. DeReggi

September 14, 1981

High Temperature Measurements of the Pyroelectric Response of PVF 2 Computers in Cardiology Conference Florence, Italy

A. S. DeReggi

September 23, 1981

Review of NBS Work on Piezoelectric Polymers \& Its Relevance to Ultrasonics

Instituto di Acustica 0.M. Corbino, Rome, Italy

A. S. DeReggi

September 28, 1981

Piezoelectric Properties of Synthetic Polymers and Their Applications

University of Pisa, Facota de Ingegneria, Pisa Italy

A. S. DeReggi

September 30, 1981

Measurement of Charge Distribution in Polymer Films

Gordon Research Conference on Polymers, Ventura, CA

A. S. DeReggi

January 11, 1982

Electric Field Distribution in Electron Beam Irradiated PVC

California Institute of Tecnology, Pasadena, CA

A. S. DeReggi

January 12,1982 
Ferroelectric Properties of PVF 2 and Copolymers and Their Potential Uses in Ultrasonics and Biomedicine Johns Hopkins University, Laure1, MD

A. S. DeReggi

February 10, 1982

High Temperature Measurements of the Pyroelectric Response of PVF 2 American Physical Society Meeting, Dallas, TX

A. S. DeReggi

March 8, 1982

Piezoelectric and Pyroelectric Properties of Synthetic Polymers and Their Applications in Transducers Southwest Research Institute, San Antonio, TX

A. S. DeReggi

March 12, 1982

Real-Time Processing of Data and Subsequent Feedback in Computer-Controlled Thermogravimetry

Eastern Analytical Symposium, New York, NY

B. Dickens

November 19, 1981

The U.S. Polymer Industry \& the Polymer Science \& Standards Division

The Institute of Chemistry, Beijing, China

R. K. Eby

September 30,1981

Structures and Disorder in the Phases of Polytetrafluoroethylene

The Institute for Chemistry, Beijing, China

R. K. Eby

October 4, 1981

The U.S. Polymer Industry \& the Polymer Science \& Standards Division

The Institute for Applied Chemistry, Changchun, China

R. K. Eby

October 6, 1981

Structures and Disorder in the Phases of Polytetrafluoroethylene

The Institute for Applied Chemistry, Changchun, China

R. K. Eby

October 7, 1981

Structures and Disorder in the Phases of Polytetrafluoroethylene Northwest University, Xian, China

R. K. Eby

October 10, 1981

Structures and Disorder in the Phases of Polytetrafluoroethylene

The Institute for Organic Chemistry, Shanghai, China

R. K. Eby

October 11, 1981

The U.S. Polymer Industry \& the Polymer Science \& Standards Division

Fu Dan University, Shanghai, China

R. K. Eby

October 12, 1981

The Structures and Disorder of the Phases of Polytetrafluoroethylene Pittsburgh Diffraction Conf., Cleveland, $\mathrm{OH}$

R. K. Eby

November 5, 1981

The U.S. Polymer Industry \& the Polymer Science \& Standards Division Indian Institute of Technology, Delhi, India

R. K. Eby

February 2, 1982

Structures and Disorders in the Phases of PTFE

University of Delhi, Delhi, India

R. K. Eby

February 4, 1982 
The U.S. Polymer Industry \& the Polymer Science \& Standards Division

National Aeronautical Laboratory, Bangalore, India

R. K. Eby

February 8, 1982

Structures and Disorders in the Phases of PTFE

National Aeronautical Laboratory, Bangalore, India

R. K. Eby

February 8, 1982

Characterization of Polymeric Materials with Emphasis on NDE for Degradation \& Failure DARPA Workshop on Nondestructive Evaluation of Polymers \& Polymer-Based Composites, Wrightsville Beach, NC

R. K. Eby

Apri1 28, 1982

Disorders in the Crystal Structures of Homo- \& Copolymers of Polytetrafluoroethylene IUPAC ' 82 Macro Conference, Amherst, MA

R. K. Eby

July 13,1982

Durability of Polymers

DARPA Matls. Res. Council Workshop on Polymer Opportunities, La Jolla, CA

R. K. Eby

July 23, 1982

Overview of the Polymer Science \& Standards Division

3M Company, Minneapolis, MN

R. K. Eby, M. G. Broadhurst, G. T. Davis, F. W. Wang, B. M. Fanconi, and I. C. Sanchez September 21, 1982

Overview of the Polymer Science \& Standards Division Kerox Corporation, Toronto

R. K. Eby, M. G. Broadhurst, G. T. Davis, F. W. Wang, B. M. Fanconi, and I. C. Sanchez September 23, 1982

Overview of the Polymer Science \& Standards Division National Research Council, Ottawa

R. K. Eby, M. G. Broadhurst, G. T. Davis, F. W. Wang, B. M. Fanconi, and I. C. Sanchez September 24, 1982

Fourier Transform Infrared Spectroscopy of Polymers. Theory \& Application Symposium on FTIR Techniques in Plastics Characterization \& Processing, ASTM, Williamsburg, VA B. M. Fanconi

March 16, 1982

Chain Scission and Mechanical Degradation of Polyethylene

IUPAC Macro ' 82 Conference, Amherst, MA

B. M. Fanconi

July 14,1982

Calculations of Defect Structure in Polytetrafluoroethylene

IUPAC Macro ' 82 Conference, Amherst, MA

B. L. Farmer

July 13,1982

Role of Thermal Analysis in the Lifetime Prediction of Polymers

Second European Symposium on Thermal Analysis, Aberdeen, Scotland

J. H. Flynn

September 3, 1981

Mettler Award Address

Royal Society of Chemistry, Salford, England

J. H. Flynn

September 7, 1981

Study of Degradation of Polymers by Thermogravimetry

Chemical Society of Vojvodina, Novi Sad, Yugoslavia

J. H. Flynn

September 15, 1981 
Comments on Two Enigmas of Condensed Phase Kinetics--The Compensation Effect and Exact Differential Rate Equations

11th North American Thermal Analysis Society Conference, New Orleans, LA

J. H. Flynn

October 21,1981

Study of the Degradation of Polymers by Thermogravimetry

Celanese Research Laboratories, Summit, NJ

J. H. Flynn

January 29, 1982

Kinetics of Polymer Degradation

Polytechnic Institute of New York, Brooklyn, NY

J. H. Flynn

May 18, 1982

Lifetime Prediction from Polymer Degradation Kinetics

IUPAC Macro ' 82 Conference, Amherst, MA

J. H. Flynn

July 15,1982

The Degradation of Polyurethanes by Thermogravimetry

7 th International Conference on Thermal Analysis, Kingston, Ontario, Canada

J. H. Flynn

August 27, 1982

Dynamic Light Scattering from Polymer Solutions

Chemistry Institute of Chinese Academy of Sciences, Beijing, China

C. C. Han

October 4, 1981

Temperature and Molecular Weight Dependence of Polymer Dimensions

Chemistry Institute of Chinese Academy of Sciences, Beijing, China

C. C. Han

October 5, 1981

Polymer Molecular Weight Characterization

Beijing National Institute of Metrology, Beijing, China

C. C. Han

October 7, 1981

Static Light Scattering Theory

Beijing National Institute of Metrology, Beijing, China

C. C. Han

October 8, 1981

Polymer Characterization of Static Light Scattering

Beijing National Institute of Metrology, Beijing, China

C. C. Han

October 9, 1981

Static Light Scattering Instrumentation-I

Beijing National Institute of Metrology, Beijing, China

C. C. Han

October 10, 1981

Static Light Scattering Instrumentation-II

Beijing National Institute of Metrology, Beijing, China

C. C. Han

October 12, 1981

Structure Factor \& Second Virial Coefficient of Polymer Solution by Light Scattering Beijing Natl. Inst. of Metrology, Beijing, China

C. C. Han

October 13, 1981

Small Angle Neutron Scattering from Polymers

Shanghai Provincial Bureau of Metrology \& Eastern Regional Measurement Center, Shanghai, China

C. C. Han

October 16, 1981 
Temperature and Molecular Weight Dependence of Polymer Dimensions

Shanghai Provincial Bureau of Metrology and Eastern Regional Measurement Center, Shanghai, China

C. C. Han

October 17, 1981

Static and Dynamic Properties of Polymer Solutions

Hongzhou Provincial Bureau of Metrology and Zhe-Jiang University, Hongzhou, China

C. C. Han

October 21, 1981

Temperature and Molecular Weight Dependence of Polymer Dimensions

Guangzhou Provincial Bureau of Metrology and Zhong-Shan University, Guangzhou, China

C. C. Han

October 23, 1981

Light Scattering \& SANS from Polymers and Polymer Solutions

Guangzhou Provincial Bureau of Metrology and Zhong-Shan University, Guangzhou, China

C. C. Han

October 24, 1981

Effects of Excluded Volume and Pre-averaging of Oseen-Tensor for Dilute Polymer Solutions by Dynamic Light Scattering

Dept. of Macromolecular Science, Osaka University, Japan

C. C. Han

November 4, 1981

Small Angle Nuetron Scattering from Polymers

UBE Industry, Osaka, Japan

C. C. Han

November 5, 1981

Dynamic Light Scattering from Dilute Polymer Solutions

Dept. of Polymer Chemistry, Kyoto University, Japan

C. C. Han

November 6, 1981

Dynamic Light Scattering from Dilute Polymer Solutions in the Intermediate q-region

Dept. of Synthetic Chemistry, Nagoya University, Japan

C. C. Han

November 9, 1981

Small Angle Neutron Scattering from Polymers

Institute of Materials Science, University of Connecticut, Storrs, CT

C. C. Han

December 4, 1981

Cooperative and Self-Diffusion of Polymers in Semidilute Solutions by Dynamic Light Scattering Dept. of Nuclear Engineering, University of Michigan, Madison, WI

C. C. Han

Apri 1 , 1982

Dynamic Light Scattering of Polymer Solutions in the Semidilute Region

Dept. of Chemistry, University of Wisconsin, Madison, WI

C. C. Han

Apri 7, 1982

Sma 11 Angle Neutron Scattering from Polymers

Dept. of Chemistry and Chemical Engineering, Virginia Polytechnic, Institute and State University,

Blacksburg, VA

C. C. Han

May 12,1982

Dynamic Light Scattering from Dilute and Semidilute Polymer Solutions

Ann Arbor Conference on Quasielastic Light Scattering Spectra \& Their Interpretations, Ann Arbor, MI

C. C. Han

June 23, 1982

Plastisol Characterization

Office of Naval Research Review on Compliant Coating Drag Reduction, Naval Research Laboratory, Washington, DC

D. L. Hunston

October 15, 1981 
Fundamental Aspects of Adhesion

Workshop on Engineering with Adhesives, East Brunswick, NJ

D. L. Hunston

October 26, 1981

Developing Failure Criteria for the Polymers Used in Structural Adhesives and Composites SPI Meeting on Reinforced Plastics/Composites, Washington, DC

D. L. Hunston

January 15, 1982

Mechanics of Adhesive Bond Failure

Center for Professional Advancement, San Mateo, CA

D. L. Hunston

February 10, 1982

Durability and Adhesive Bond Failure

Center for Professional Advancement, San Mateo, CA

D. L. Hunston

February 10, 1982

Comparison Between Fracture Behavior in Adhesive Bonds \& Fibrous

Composites Adhesion Society Meeting, Mobile, AL

D. L. Hunston

February 23, 1982

Mechanical Properties and Failure Behavior of Toughened Glassy Polymers

Symposium, Civil Engineering Dept., Texas A\&M University, College Station, TX

D. L. Hunston

February 25, 1982

Fracture Behavior of Adhesives and Composite Matrix Resins

General Dynamics Research Laboratories, Ft. Worth, TX

D. L. Hunston

February 26, 1982

Crack Propagation in Rubber-Toughened Epoxy

International Conf. on Deformation, Yield \& Fracture, Cambridge, England

D. L. Hunston and A. J. Kinloch

March 31, 1982

General Rheology and Its Applications in Adhesion

Workshop on Adhesion and Adhesives, Kent State University, Kent, $\mathrm{OH}$

D. L. Hunston

June 7, 1982

Flow Fundamentals in Fluid Systems Containing High Molecular Weight Additives

Symposium on Applied Rheology for the Industrial Chemists, Kent State Univ., Kent, OH

D. L. Hunston

June 24,1982

Fracture Behavior of Brittle Materials

Symposium on Applied Rheology for the Industrial Chemist, Kent State University, Kent, $O H$ D. L. Hunston

June 25,1982

Cure Monitoring of Thermosetting Polymers by Ultrasonics

AF-NAVY/DARPA Review on Quantitative NDE, San Diego, CA

D. L. Hunston

August 6,1982

Characterization of Fracture Behavior of Adhesive Joints

ACS International Symp. on Adhesive Joints, Kansas City, MO

D. L. Hunston

September 15, 1982

Mechanics of Fracture for Adhesive Joints

ACS International Symposium on Adhesive Joints, Kansas City, MO

D. L. Hunston and S. S. Wang

September 15, 1982 
Rheology of Cure for Intaglio Printing Inks

ACS Symposium on Chemorheology of Thermosetting Polymers, Kansas City, Mo

D. L. Hunston

September 16, 1982

The Morphology of Polymers

31st Annual Meeting, Society of Polymer Science of Japan, Tokyo, Japan

F. A. Khoury

May 30, 1982

The Morphology of Polyethylene Crystals Grown from Solution at High Temperatures, Kyoto University, Kyoto, Japan

F. A. Khoury

June 2, 1982

The Crystallization and Morphology of Polymers

Osaka University, Osaka, Japan

F. A. Khoury

June 3, 1982

The Morphology of Polymer Crystals Grown From Solution

UBE Plastics Laboratory, Hirakata, Japan

F. A. Khoury

June 4, 1982

The Morphology of Polymer Crystals Grown from Solution

Kyushu University, Fukuoka, Japan

F. A. Khoury

June 8, 1982

Dynamic Light Scattering in the Intermediate Momentum Transfer Region

National Institutes of Health, Bethesda, MD

T. P. Lodge

November 19, 1981

Conformation and Dynamics of Polymers in Solution

University of Iowa, Iowa City, IA

T. P. Lodge

January 18, 1982

Conformation and Dynamics of Polymers in Solution

University of Pittsburgh, Pittsburgh, PA

T. P. Lodge

January 20, 1982

Conformation and Dynamics of Polymers in Solution Carnegie-Melon University, Pittsburgh, PA

T. P. Lodge

January 21, 1982

Conformation and Dynamics of Polymers in Solution University of Georgia, Athens, GA

T. P. Lodge

January 26, 1982

Conformation and Dynamics of Polymers in Solution Georgia Institute of Technology, Atlanta, GA

$T$. P. Lodge

January 28, 1982

Conformation and Dynamics of Polymers in Solution Purdue University, West Lafayette, IN

T. P. Lodge

February 1, 1982

Conformation and Dynamics of Polymers in Solution Dartmouth College, Hanover, NH

T. P. Lodge

February 8, 1982 
Conformation and Dynamics of Polymers in Solution University of Illinois, Urbana, IL

T. P. Lodge

February 12, 1982

Conformation and Dynamics of Polymers in Solution

Cornell University, Ithaca, NY

T. P. Lodge

February 17, 1982

Conformation and Dynamics of Polymers in Solution University of Michigan, Ann Arbor, MI

T. P. Lodge

February 19, 1982

Conformation and Dynamics of Polymers in Solution

University of Minnesota, Minneapolis, MN

T. P. Lodge

February 22, 1982

Conformation and Dynamics of Polymers in Solution

University of California, Berkeley, CA

T. P. Lodge

February 24, 1982

Dynamic Light Scattering in the Intermediate Momentum Transfer Region

APS Meeting, Dallas, TX

T. P. Lodge

March 11, 1982

Oscillatory Flow Birefringence Properties of Polymer Solutions at High Effective Frequencies

IUPAC Macro ' 82 Conference, Amherst, MA

T. P. Lodge

July 13, 1982

Non-linear Viscoelastic Behavior of PMMA in Torsion

Centre de Recherches sur les Macromolecules, Strasbourg, France

G. B. McKenna

November 10, 1981

Le Compartement Viscoelastique du PMMA sous Torsion

L'Ecole d'Applications des Hauts Polymeres Universite Louis Pasteur, Strasbourg, France

G. B. McKenna

March 10, 1982

On the Small Strain Behavior of Crosslinked Natural Rubber in Torsion, Extension and Compression Malaysian Rubber Producers Research Assn., Brickendonbury, England

G. B. McKenna

Apri 1 2, 1982

La Variation due Temps a la Rupture du PMMA et du Polyethylene

L'Ecole Polytechnique Federale de Lausanne, Lausanne, Switzerland

G. B. McKenna

Apri1 30, 1982

Deviations from Elasticity Theory of Rubber Behavior at Small Strains

6th European "Network-Group" Mtg., Strasbourg, France

G. B. McKenna

July 1, 1982

Adsorption on Hydroxyapatite

Chemistry Dept., Lucknow University, Lucknow, India

D. N. Misra

November 10,1981

Precision Time Domain Measurements for Audio Frequencies and Below

Gordon Research Conference on Dielectrics, Plymouth, NH

F. I. Mopsik

August 2, 1982 
The Role of Dental Materials in Dental Practice

Dept. of Materials Science, Univ. of Virginia, Charlottesville, VA

G. C. Paffenbarger

December 3, 1981

Transport Properties of Highly Drawn Semicrystalline Polymers

Fiber Society Symp., Princeton University, Princeton, NJ

A. Peterlin

May 5, 1982

Mechanical Properties \& Morphology of Drawn Semicrystalline Polymers

IUPAC ' 82 Meeting, Amherst, MA

A. Peterlin

July 14, 1982

History of Polymer Physics

Gordon Research Conferences, Andover, NH

A. Peterlin

July 20,1982

Transport Properties as an Extremely Sensitive Indicator of the Status of the Amorphous Component in the Elastically and Plastically Deformed Semicrystalline Polymer

IUPAC Symposium on Polymers, Athens, Greece

A. Peterlin

August 31,1982

Nature of Fold Surfaces in Lamellar Crystals

European Conference on Polymer Physics, Barcelona, Spain

A. Peterlin

September 22, 1982

Brownian Motion and Polymer Chain Adsorption

Dartmouth College, Hanover, $\mathrm{NH}$

R. Rub in

October 2, 1981

Some Random Walk Questions: Haven't We Been Here Before?

Statistical Physics Seminar, University of Maryland, College Park, MD

R. Rubin

February 16, 1982

Some Far Out Properties of Adsorbed Polymer Chains

University of California, San Diego, La Jolla, CA

R. Rubin

September 1, 1982

Finishing Amalgams

Prevention of Microleakage \& Finishing Composite Restorations, Naval Dental School, Bethesda, MD

N. W. Rupp

September 2, 1981

Esthetic Restorative Materials: Third Generation

Wright-Patterson Air Force Base, Dayton, $\mathrm{OH}$

N. W. Rupp

October 5, 1981

Selection and Use of Dental Materials

Eglin Air Force Base, FL

N. W. Rupp

November 10, 1981

The Selection and Use of Dental Materials

Fresno Madera Dental Foundation, Fresno, CA

N. W. Rupp

December 11, 1981

Research in the Development of Dental Specifications Georgetown University Dental School, Washington, DC

N. W. Rupp

January 13, 1982 
Proper Manipulation of Dental Materials

Veterans Administration Hospital, Washington, DC

N. W. Rupp

January 14, 1982

Dental Cements and Impression Materials

Naval Graduate Dental School, Bethesda, MD

N. W. Rupp

January 28, 1982

Dental Materials for the Fixed Prosthodontist

Veterans Administration Hospital, Washington, DC

N. W. Rupp

February 8, 1982

Dental Research Activities at the National Bureau of Standards

Dental Hygienists' Association, Wilmington, DE

N. W. Rupp

February 16, 1982

Finishing the Amalgam Restoration

Academy of Operative Dentistry, Chicago, IL

N. W. Rupp

February 18, 1982

Impression and Cementation Materials

Naval Dental School, Bethesda, MD

N. W. Rupp

March 1, 1982

Cementation Materials

Georgetown University Dental School, Washington, DC

N. W. Rupp

March 4, 1982

Selection and Use of Dental Materials

Lackland Air Force Base, TX

N. W. Rupp

March 16, 1982

Materials for the Laboratory Technician

Veterans Administration Hospital, Washington, DC

N. W. Rupp

March 23, 1982

Esthetic Restorative Materials

Georgetown University Dental School, Washington, DC

N. W. Rupp

March 25, 1982

Dental Amalgam

Georgetown University Dental School, Washington, DC

N. W. Rupp

Apri 1 1, 1982

The Selection and Use of Restorative Dental Materials: Direct and Indirect Techniques Research Study Club of Nebraska, Lincoln, NE

N. W. Rupp

May 21, 1982

Clinical Significance of Pulp Studies and the Biological Responses to Dental Restorative Procedures and Materials

West Central District Dental Society's 70th Annual Meeting, Brainerd, MN

N. W. Rupp

June 12,1982

Theory of Phase Equilibria in Polymer Blends

Rutgers University, Piscataway, NJ

I. C. Sanchez

October 9, 1981 
Theory of Interfacial Tension in Polymer Mixtures

Kodak Research Laboratories, Rochester, NY

I. C. Sanchez

December 9, 1982

Theory of Polymer Blend Phase Behavior

4 th Annual Polymer Summer School, Yugoslav Academy of Sciences,

Dubrovnik, Yugoslavia

I. C. Sanchez

April 26, 1982

Thermodynamic Properties of Polymer Blends

Polymer Gordon Research Conference, Colby-Sawyer College, New London, NH I. C. Sanchez

July 5, 1982

Thermodynamic Properties of Polymer Blends

IUPAC Macro ' 82 Conference, Amherst, MA

I. C. Sanchez

July 12,1982

Theory of Polymer Interfacial Tension

Gordon Research Conference on the Science of Adhesion, New Hampton, NH

I. C. Sanchez

August 23, 1982

Measurement of Polymer-Solvent Diffusivity by Inverse Gas Chromatography Princeton University, Princeton, NJ

G. A. Senich

Apri1 10, 1982

What Polymer Chemists Do at the National Bureau of Standards

Howard University, Washington, DC

G. A. Senich

October 7, 1981

Government Careers in Polymer Research at the National Bureau of Standards George Washington University, Washington, DC

G. A. Senich

October 21, 1981

Characterization of Polymers: Mechanical Property and Diffusion Studies NASA Langley Research Center, Hampton, VA

G. A. Senich

October 26, 1981

Gas Chromatography of Polymers

Chemical Society of Washington, Rockville, MD

G. A. Senich

February 11, 1982

Chromatographic Studies of Diffusion in Polymers

IUPAC Macro ' 82 Conference, Amherst, MA

G. A. Senich

July 14,1982

Measurement of Additive Migration from Plastic Food Packaging

ISO Consumer Policy Council, Paris, France

L. E. Smith

Apri门 27, 1981

Models for Migration of Additives from Polyolefins

ASTM Technical Seminar, Williamsburg, VA

L. E. Smith

March 16, 1982 
Castability and Fit of Nonprecious Alloys

Eastern Maryland Dental Society, Ocean City, MD

J. A. Tesk

October 2, 1981

Applications of ${ }^{13} \mathrm{C}$ NMR to the Characterization of Solid Polymers

Virginia Polytechnic Institute, Blacksburg, VA

D. L. VanderHart

October 1, 1981

NMR Studies of Oriented Polymers, LPE and PET

Institute fur Physikalische Chemie, University of Mainz, Germany

D. L. VanderHart

March 22, 1982

${ }^{13} \mathrm{C}$ NMR Studies in Solid Polymers

University of East Anglia, Norwich, England

D. L. VanderHart

March 24, 1982

NMR Studies of Oriented Polymers, LPE, and PET

University of Leeds, Leeds, England

D. L. VanderHart

March 26, 1982

${ }^{13} \mathrm{C}$ NMR of Drawn Polymers

University of Bristol, Bristol, England

D. L. VanderHart

March 29, 1982

High Resolution ${ }^{13} \mathrm{C}$ NMR of Solid Polymers

Annual Chemical Congress of the Royal Soc. of Chemistry, Birmingham, England

D. L. VanderHart

Apri 1 2, 1982

${ }^{13} \mathrm{C}$ NMR in Oriented Polymers

IUPAC Macro ' 82 Conference, Amherst, MA

D. L. VanderHart

July 12,1982

Inferences About Molecular Motion in Solid Polymers from Proton-Decoupled ${ }^{13} \mathrm{C}$ NMR

NMR Minisymposium, Monsanto Research Center, St. Louis, MO

D. L. VanderHart

July 20, 1982

NMR in Solid Polymers--An Overview

24th Rocky Mountain Conference, Society for Applied Spectroscopy, Denver, CO

D. L. VanderHart

August 3, 1982

Measurement of Polymer-Polymer Compatibility by Non-Radiative Energy Transfer Technique

Gordon Research Conference on the Science of Adhesion, New Hampton, NH

F. W. Wang

August 25, 1982

Spectroscopic Study of Amorphous Polyethylene

Johnson \& Johnson Co., Milltown, NJ

S. L. Wunder

September 15, 1981

Conformational and Morphological Properties of Polymethylenes

International Mineral and Chemical Co., Terre Haute, IN

S. L. Wunder

November 9, 1981

Conformational and Morphological Properties of Polymethylenes

Hercules, Wilmington, DE

S. L. Wunder

November 12, 1981 
Conformational and Morphological Properties of Polymethylenes

Exxon Research and Engineering Co., Lindberg, NJ

S. L. Wunder

November 17,1981

Conformational and Morphological Properties of Polymethylenes

Aerojet Strategic Prop., Sacramento, CA

S. L. Wunder

December 9, 1981

Conformational and Morphological Properties of Polymethylenes

E. I. duPont Co., Wilmington, $D E$

S. L. Wunder

December 14, 1981

Interatomic Distances in A15 Compounds: Geller vs Pearson Hypotheses

Los Alamos National Laboratory, Los Alamos, NM

R. M. Waterstrat

October 7, 1981

Wear Mechanisms in Dental Composites

American Association of Dental Schools, New Orleans, LA

W. Wu

March 16, 1982

Non-Linear Mechanical Behavior of Polymer Solutions at Various Concentrations

IUPAC Macro ' 82 Conference, Amherst, MA

L. J. Zapas

July 15,1982

Seminars for Staff and Guests

Recent Advances in Polyacetylene Chemistry

Professor Hideki Shirakawa

University of Tsukuba, Tokyo, Japan

August 24, 1981

Mechanisms of Rubber Adhesion and Friction

A. D. Robert

Malaysian Rubber Producers' Research Association, Hertford, England

August 27, 1981

Materials Used in the Lithographic Process

Or. Larry Thompson

Bell Laboratories, Murray Hill, NJ

September 23, 1981

Polymer Impregnation and Microstructure of Hardened Cement Pastes of Various Porosities Raouf S. Mikhail

Ain Shams University, Cairo, Egypt

September 28, 1981

Disorder in Chain Molecules Observed with Vibrational Spectroscopy

Or. R. G. Snyder

University of California, Berkeley, CA

November 3, 1981

Scattering Studies of Blends

Professor Richard S. Stein

University of Massachusetts, Amherst, MA

November 19, 1981

On the Role of Liquid Elasticity in Polymer Characterization and Journal Bearing Lubrication

A. S. Lodge

University of Wisconsin, Madison, WI

November 24, 1981

Clinical-Pathologic Issues in Cardiac Valve Prostheses and Ventricular Assist Devices

Dr. Fred J. Schoen

Peter Brigham and Women's Hospital, Boston, MA

December 10, 1981 
Renormalization Calculation of the Structure Factor for Polymers

Professor T. Witten

University of Michigan, Ann Arbor, MI

December 12, 1981

Mechano-vibrational Spectroscopy of Polymers

Professor Shaw Ling Hsu

University of Massachusetts, Amherst, MA

December 14, 1981

Time Temperature Equivalence: After All These Years Can It Be Wrong?

Dr. N. G. McCrum

University of Oxford, Oxford, England

March 16, 1982

Mechanism of Physical Aging in Crystalline Polymers

Dr. N. G. McCrum

University of Oxford, Oxford, England

March 16, 1982

Effect of Thermal Parameters on Compatibility in Porcelain Veneered Split Metal Rings Dr. G. E. O. Widera

University of Illinois, Chicago, IL

March 18, 1982

Small Angle Neutron Scattering of a Rubber Network

Dr. Robert UIIman

Ford Motor Company, Dearborn, MI

March 29, 1982

Yield and Fracture of Model Polyethylene Systems

Dr. David C. Bassett

University of Reading, England

March 29, 1982

Crystallization of Polyethylene Copolymers

Dr. David C. Bassett

University of Reading, England

March 30,1982

Contact Electrification of Polymer Dielectrics \& Properties at High Electric Fields

Professor G. Fuhrmann

University of Kaiserslautern, Germany

April 1, 1982

The True Biodegradation of Implanted Polymers

Dr. David F. Williams

University of Liverpool, England

April 16, 1982

Polymers for Enhanced 0il Recovery

Dr. L. Guy Donaruma, Professor of Chemistry

Polytechnic Inst. of New York, New York, NY

April 21, 1982

Changes in the Elastic Modulii of Rubber Due to Low Temperature Crystallization

Dr. Andrew Stevenson

The Malaysian Rubber Producers' Research Association, Brickendonbury, Hertford, England

April 22, 1982

Conformational and Long Branch Analysis by GPC \& Laser Light Scattering

Dr. Roger S. Porter

University of Massachusetts, Amherst, MA

May 5, 1982

Polymer Liquid Crystals/Biaxial Orientation and Its Development in Polymer Systems Professor James L. White

University of Tennessee, Knoxville, TN

May 6, 1982 
Synthesis and Characterization of Model Macromolecules

Professor L. J. Fetters

Institute of Polymer Science, University of Akron, Akron, $\mathrm{OH}$

May 10, 1982

The Synthesis of Hydrophilic Polymers with Nucleic Acid Base Side Chains

Dr. Charles G. Overberger

University of Michigan, Ann Arbor, MI

May 14, 1982

Sliding-Wear Behavior of Dental Restoratives

Dr. William F. Bailey

University of Connecticut, Storrs, CT

May 20, 1982

Influence of Molecular Weight Distribution and Catalysts on the Crystallization and Orientation of Polyethylene Terephthalate

Dr. H. G. Zachmann

Martin-Luther-King Platz 6, Hamburg, West Germany

May 21, 1982

Miscibility Behavior of Polyester/Halogenated Polymer Blends

Dr. R. E. Prud'homme

Universite Laval, Quebec, Canada

June 17, 1982

The Oxidation of Stabilized Polypropylenes

Dr. Frank Mayo

Stanford Research Inst., Stanford, CA

June 18, 1982

Infiltration of Monomers into Hard Tissues

Dr. N. Nakabayashi

Tokyo Medical and Dental University, Tokyo, Japan

June 21, 1982

Kineto Rheology, Chemical Change and Rheological Response

Professor Alex Silberberg

The Weizmann Institute of Science, Rehovot, Israel

July 2, 1982

Fine Structures of Fibers and Films Made from a Liquid Crystal

Dr. M. Horio

Hirakata Plastics Lab., Osaka, Japan

July 7, 1982

Forced Rayleigh Light Scattering of Polymers in Semi-Dilute Solution \& Melts

Madam Liliane Leger

Laboratoire de Physique de la Matiere Condensee, College de France, Cedex, France

July 7, 1982

Photovoltaic Effect in Ferroelectric Polyvinylidene Fluoride Film

Professor H. Sasabe

Tokyo University of Agriculture and Tech., Tokyo, Japan

July 8, 1982

Rheo-Optical Properties of Poly-Alpha-0lefins

H. Kawai

Kyoto University, Kyoto, Japan

July 9,1982

Qualitative and Quantitative Information on Linear Polyethylene Fractions as a Function of Molecular Weight

Dr. Ingrid Voigt-Martin

Mainz University, Inst. fur Physikalische, West Germany

July 9, 1982

Microphase Separated Structure of Block Copolymers and Its Related Properties

Professor Takeji Hashimoto

Kyoto University, Kyoto, Japan

July 9, 1982 
Time-Dependent Nonlinear Rheological Properties of Entangled Polymer Melts and Concentrated Solutions Professor David Soong

University of California, Berkeley, CA

July 19,1982

Polarization Switching \& Molecular Motion in PVDF \& Related Copolymers

Professor A. Odajima

Hokkaido University, Sapporo, Japan

July 20,1982

Electron Microscopy of Polyvinylidene Fluoride

Professor K. Sakaoku

University of Tokyo, Tokyo, Japan

July 21, 1982

Luminescent Probe Study on Reactions and Chain Dynamics of Polymers

Professor I. Mita

Faculty of Engineering, Univ. of Tokyo, Tokyo, Japan

July 21,1982

Gas Liquid Chromatographic Studies on Polymeric Stationary Phases

Professor D. D. Deshpande

Indian Institute of Technology, Bombay, India

July 22, 1982

Thermoluminescence from Irradiated Polymers and Fibers

Or. T. Sakai

Tokyo Institute of Technology, Tokyo, Japan

July 22, 1982

${ }^{1} \mathrm{H}$ and ${ }^{13} \mathrm{C}$ NMR Studies of Solid Polymers

Dr. K. J. Packer

School of Chemical Sci., University of East Anglia, England

July 23, 1982

Mechanical Properties \& Morphology of Fiber Glass Reinforced Polypropylene Composites Professor A. Misra

Indian Institute of Technology, New Delhi, India

July 23, 1982

Dynamic Behavior of Polyelectrolytes in Solution

Professor Y. Wada

Yamagata University, Yonezawa, Japan

July 26, 1982

Novel Imaging Techniques for Phase Separated Polymers

Professor N. Thomas

University of Massachusetts, Amherst, MA

August 11, 1982

Piezoelectricity in Vinylidene Cyanide-Vinyl Acetate Copolymers

Professor S. Miyata

Tokyo University, Tokyo, Japan

September 8, 1982

Chemical Characterization of Solid Cools via Magic Angle ${ }^{13} \mathrm{C}$ NMR

Dr. John Havens

Case Western Reserve University, Cleveland, $\mathrm{OH}$

September 15, 1982

NDE for Polymer Composites

Professor R. D. Adams

University of Bristol, Bristol, England

September 22, 1982

Technical and Professional Committee Participation and Leadership

Academy of Operative Dentistry

N. W. Rupp, Councilor 
American Association for Dental Research/International Association for Dental Research

R. L. Bowen

Member: Dental Materials Group

Wilmer Souder Award Committee

J. M. Antonucci

Member: Dental Materials Group

G. M. Brauer

Member: Dental Materials Group

J. M. Cassel

Member: Dental Materials Group

J. E. McKinney

Member: Dental Materials Group

W. Wu

Member: Dental Materials Group

J. A. Tesk

Member: Dental Materials Group

W. E. Brown

Member: Biological Mineralization Research Award Committee Mineralized Tissue Group, Councilor

American Association of Dental Research/Washington Section

J. A. Tesk

Secretary-Treasurer

American Chemical Society

J. M. Antonucci

Member: Board of Managers

Advertising Editor

G. M. Brauer

Member: Meetings and Expositions Committee Councilor

D. N. Misra

Chairman: Symposium on Adsorption on and Surface Chemistry of Hydroxyapatite, Kansas

City, MO, Sept. 1982

American Dental Association Special Committee on the Future of Dentistry

R. L. Bowen

Member: Research Working Group

American Institute of Mining, Metallurgical, and Petroleum Engineers

R. M. Waterstrat

Member: Committee on Alloy Phases

American Institute of Physics

R. K. Eby

Chapter Editor, Physics Handbook

H. P. R. Frederikse

Chapter Editor, Physics Handbook

E. A. Kearsley

Member: Committee for Public Policy

American National Standards Committee, M0156, Dental Materials, Instruments, and Equipment

R. L. Bowen

Member: Subcommittee on Biological Evaluation of Dental Materials, Secretary

G. M. Brauer

Member: Government Representative

Denture Resins, Chairman

Z-E Type Material 
J. M. Cassel

Member: C054, Medical Devices Standards Management Board, Executive Committee

N. W. Rupp

Member: Alloy for Dental Amalgams, Chairman

Dispensers for Alloys and Mercury, Chairman

Mercury for Dental Amalgam, Chairman

Mechanical Amalgamators, Chairman

J. A. Tesk

Member: Base Metal Alloys

Dental Implants, Secretary

Dental Materials, Instruments, \& Equipment, Chairman

Porcelain/Alloy Systems

USA TAG for ISO TC106, Chairman

American Physical Society

Division of High Polymer Physics

F. A. Khoury

Member: Executive Committee

Committee on History of DMPP

I. C. Sanchez

Member: Executive Committee, Vice Chairman, 1982-83

Selection Committee, APS High Polymer Physics

Prize, $1982-83$

Education Committee, Chairman, 1982

American Society of Mechanical Engineers

R. W. Penn

Member: Reinforced Thermoset Plastic Corrosion Resistant Equipment

American Society for Testing and Materials

Committee on Research and Technical Planning

E. Passaglia

Member: Subcommittee on Research Chairman

D-2: Petroleum Products and Lubricants

L. J. Zapas

Member: 02.07 Flow Properties

D2.07A Newtonian Viscometry

D-9: Electrical Insulating Materials

W. P. Harris

Member: 09.05 Ceramic Products

09.12 Electrical Tests

09. 16 Hookup Wire Insulation

09.17 Thermal Capabilities

09.90 Executive Committee

09.93 Education, Research, and Symposia

09.94 Nomenclature, Significance, \& Statistics

09.95 Liaison

D-11: Rubber and Rubber--Like Materials

E. A. Kearsley

Member

G. B. McKenna

Member

D-20: Plastics

R. K. Eby, Chairman

Member: D20.01: Executive Subcommittee

J. D. Barnes

Member: D20.12: 0lefin Plastics

D20.13: Statistics, Chairman

D20.19: Film and Sheeting

D20.70: Analytical Methods

WG.07: Gas Permeability 
H. L. Wagner

Member:

WG. 04 Liquid Size Exclusion Chromatography

WG.05 Molecular Weight Parameters, Chairman

J. H. Flynn

Member: D20.30: Thermal Properties

30.7 Fundamental Thermal Methods, Chairman

D-27: Electrical Insulating Liquids and Gases

W. P. Harris

Member

D-30: High Modulus Fibers and Their Composites

D. L. Hunston

Member

E-9: Fatique

G. B. McKenna

Member

R. W. Penn

Member

E-37: Thermal Measurements

S. S. Chang

Member: E37.01: Test Methods and Recommended Practices

E37.05: Thermophysical Properties

J. H. Flynn

Member: E37.03: Nomenclature and Definitions, Chairman

E37.90: Executive Subcommittee

E-45: Geothermal Energy and Resources

E. A. Kearsley

Member: E45.02: Materials

F-2: Flexible Barrier Materials

J. D. Barnes

Member: F02.30: Test Methods

F02.93: Statistical Matters, Chairman

WG. 30.2: Barrier Methods, Chairman

F-4: Medical and Surgical Materials, and Devices

G. M. Brauer

Member: Executive Committee

F04.20: Resources

WG.01: Polymeric Materials, Chairman

J. M. Cassel

Member: F04.20: Resources

R.K. Eby

Member

G. B. McKenna

Member

Biomedical Research Support Grant

R. L. Bowen

Member: 1982-1983 Review Committee

W. E. Brown

Member: 1982-1983 Review Committee

Case Western Reserve Univ Materials Research Laboratory

Advisory Board

R. K. Eby

Member 
Chemical Society of Washington

G. M. Brauer

Chairman, Councilors Committee

Award Committee

DECUS (Digital Equipment Users' Society)

Washington Area MIN-LSI Users'

J. D. Barnes

Chairman

Eleventh NATAS Conference

J. H. Flynn

Symposium on Kinetics of Chemical \& Physical Processes, Chairman

Ferroelectrics Journal

M. G. Broadhurst

Guest Editor of Special Issue on PVDF

Fifth International Meeting on Ferroelectrics

M. G. Broadhurst

Member: U. S. Committee

Polymer Session Chairman

Geis Foundation for the Advancement of Dentistry

G. C. Paffenbarger

President

Member: Award Committee for Dental Editorial, Chairman Award Committee for Oral \& Maxillofacial Surgery, Chairman

Award Committee for Periodomtology, Chairman

Government Interagency Panel on Adhesive Bonding Research

D. L. Hunston

Member

International Confederation for Thermal Analysis

J. H. Flynn

Member: Nomenclature Committee

Working Party on Symbols

International Council on Alloy Phase Diagrams

R. M. Waterstrat

Member

International Electrotechnical Commission

TC046, Cables, Wires, \& Waveguides for Telecommunication

Equipment

L. J. Zapas

Member: WG.03: Measuring Methods of Polyethylene

TC061, Plastics

L. E. Smith

Member

International Standards Organization

TC106, Dental Materials and Devices

G. M. Brauer

Member: Task Force on Color Stability

N. W. Rupp

Member: Restorative Materials

WG. 01: U. S. Delegation, Chairman

TG7: Alloys for Dental Amalgams,

Chairman

WG.06: Amalgamation Devices \& Accessories, Chairman

J. A. Tesk, USA Delegation, Chairman

TC150, Implants for Surgery, USTAG

J. M. Casse 1, Chairman 
TC150/SC2, Cardiovascular Implants for Surgery, USTAG

J. M. Cassel, NBS Representative

Molecular and Cellular Thermobiology (Journal)

B. Dickens

Member: Editorial Board

National Bureau of Standards Advisory Panel for Tariffs on Electron Microscopes Under Florence Agreement

F. A. Khoury, Member

National Center for Small Angle Scattering

C. C. Han

Member: Users Committee

National Health and Medical Research Council of Australia

G. C. Paffenbarger, Consultant

National Institute of Dental Research

R. L. Bowen

Member: Long Range Research Planning \& State-of-the-Science on Composites, Consultant

Panel on Restorative Materials Program

Review Committee for ADA

National Research Council

Panel on Polymer Science \& Engineering

R. K. Eby, Member

Panel on Civil/Navy Ocean Engineering

Task Group on Materials and Structure

Nondestructive Testing of NRC

R. K. Eby, Member

North American Thermal Analys is Society

J. H. Flynn

Editor: NATAS Notes

Member: Awards Committee

Executive Committee

Publications Committee, Chairman

Ohio Dental Association

G. C. Paffenbarger

Member: Callahan Memorial Committee

Polymer (Journal)

R. K. Eby, U. S. Associate Editor

Polymer Degradation and Stablility (Journal)

J. H. Flynn, Editorial Board Member

Seventh International Conference on Thermal Analysis

J. H. Flynn

Member: Program Committee

Chairman: Session of Polymer Science Section

Society of Plastics Engineers

R. K. Eby

Member: Selection Committee for SPE International Award for Research, Chairman

G. B. McKenna

Member: Technical Publications

Society of Rheology

D. L. Hunston

Member: Executive Committee

E. A. Kearsley

Member: Executive Committee

G. B. McKenna

Member: Committee on Education

Local Arrangements 1981 Spring Meeting, Chairman 
Symposium on FTIR Techniques in Plastics Characterization and Processing, Williamsburg, VA,

March 18, 1982.

J. D. Barnes, Chairman

U.S. Air Force

National Civilian Consultant to the Surgeon General

N. W. Rupp

US/Yugoslav Science and Technology Program

Thermal Degradation of Polymers

J. H. Flynn, Monitor

University of Virginia, Department of Materials Science

G. C. Paffenbarger, Consultant

University of Massachusetts Advisory Board of Polymer Research Institute

R. K. Eby, Member

Washington Area MINC Users Group

J. D. Barnes, Chairman

Younger Chemists Committee

G. A. Senich

Member: Activities Committee

\section{Publications}

Antonucci, J. M.; Misra, D. N.; Peckoo, R. J. The accelerative and adhesive bonding capabilities of surface-active accelerators. J. Dent. Res. 60(7): 1332; 1981.

Bowen, R. L. Historical development of dental composite resins. Dent. Diamond (Jpn) 6: 8; 1981.

Brauer, G. M.; Dulik, D. M.; Hughes, H. N.; Dermann, K.; Rupp, N. W. Marginal adaptation of BIS-GMA-based composites containing various diluents. J. Dent. Res. 60(12): 1966; 1981.

Brauer, G. M.; Stansbury, J. W.; Antonucci, J. M. 4-N,N-dialkylaminophenethanols,-alkanoic acids and esters: new accelerators for dental composites. J. Dent. Res. 60(7): 1343; 1981.

Broadhurst, M. G. ; Micheron, F.; Wada, Y. Guest editorial. Ferroelectr. J. April 1981.

Broadhurst, M. G. Workshop Proceedings: Morphology of polyethylene and crosslinked polyethylene. EPRI Contract TPS 81-184; November 1981.

Broadhurst, M. G.; Davis, G. T. Ferroelectric polarization in polymers. Ferroelectrics 32: 177; 1981.

Bur, A. J. Resistivity and piezoelectric measurements in oriented \& unoriented thick PVDF films. Polymer 22: $1288 ; 1981$.

Bur, A. J.; Tsao, A. K. Fabrication of ultra-drawn thick PVDF transducers. Nat. Bur. Stand. (U.S.) NBSIR 81-2418; 1981 December.

Cassel, J. M.; Tesk, J. A.; Brauer, G. M.; Antonucci, J. M.; Wu W. Properties \& interactions of oral structures \& restorative materials. Nat. Bur. Stand. (U.S.) NBSIR 81-2431; 1981 December.

Chang, S. S. Specific heat of thermosetting resins--study of phenolic resin by automated adiabatic calorimetry and differential scanning calorimetry, chapter in Thermal analysis in polymer characterization. Turi, E. A., ed. London, England: Heyden \& Sons; 1981. p. 98.

Chang, S. S.; Smith, L. E.; Senich, G. A. Migration of low molecular weight additives in polymers. Nat. Bur. Stand. (U.S.) NBSIR 81-2314; 1981 September.

Chiang, C. K. An all--polymeric solid state battery. Polymer 22: 1454; 1981.

Chiang, C. K.; Franklin, A. D. Electrical impedance spectra of tran-polyacetylene. Solid State Comm. 40: $775 ; 1981$.

Chow, L. C.; Guo, M. K.; Hsieh, C. C.; Hong, Y. C. Apatitic fluoride increase in enamel from a topical treatment involving intermediate $\mathrm{CaHPO}_{4} \cdot 2 \mathrm{H}_{2} \mathrm{O}$ formation, an in vivo study. Caries Res. 15: 369; 1981.

Crissman, J. M.; Khoury, F. A. ; McKenna, G. B. Relationships between morphology \& mechanical properties of ul tra high molecular weight polyethylene. Nat. Bur. Stand. (U.S.) NBSIR 82-2493; 1981. 
Dickens, B. Pressure as an important variable in thermogravimetric studies of polymer degradations. Dollimore, D., ed. Proceedings of the 2nd european symposium on thermal analysis; 1981 September; Aberdeen, Scotland. London, England: Heyden \& Sons. 1981. p. 219.

Eby, R. K.; Broadhurst, M. G.; Chang, S. S.; Davis, G. T.; DiMarzio, E. A. ; Fanconi, B. M. ; Flynn, J. H. ; Han, C. C.; Khoury, F. A. ; Markovitz, H.; McKinney, J. E.; Mopsik, F. I.; Passaglia, E.; Sanchez, I. C. ; Smith, L. E.; VanderHart, D. L.; Zapas, L. P. High polymer physics, chapter in American Institute of Physics 50th Anniversary Handbook. Anderson, H. L., ed. New York, NY: American Institute of Physics; 1981.

Eby, R. K.; Peterlin, A.; Khoury, F. A.; Broadhurst, M. G. ; Fanconi, B. M. Crystalline polymers, chapter in State of polymer science in the U.S. National Research Council; 1981.

Fanconi, B. M.; DeVries, K. L.; Smith, R. H. Response to commentary by M. Dole. Polymer 22: 1460; 1981.

Farmer, B. L.; Eby, R. K. Energy calculations of the crystal structure of the low temperature phase (II) of polytetrafluoroethylene. Polymer 22: 1487; 1981.

Flynn, J. H. Role of thermal analysis in the lifetime prediction of polymers. Dollimore, D., ed. Proceedings of the 2nd European symposium on thermal analysis; 1981 September; Aberdeen, Scotland. London, England: Heyden \& Sons; 1981. p. 223.

Flynn, J. H. Analysis of thermogravimetric kinetics--overcoming complications of thermal history, chapter 3 in Thermal analysis in polymer characterization. London, England: Heyden \& Sons; 1981.

Han, C. C.; Akcasu, A. Z. On the dynamic structure factor, $S(q, w)$ of polymers in dilute solutions. Polymer 22: 1019; 1981.

Han, C. C.; Akcasu, A. Z. Concentration dependence of diffusion coefficient of various molecular weights and temperatures. Polymer 22: 1165; 1981.

Han, C. C.; Akcasu, A. Z. Dynamic light scattering of dilute polymer solutions in the non-asymptotic q-region. Macromolecules 14: 1080; 1981.

Hunston, D. L.; Bitner, J. L. ; Rushford, J. L.; Rose, W. S.; Riew, C. K. Viscoelastic fracture of structural adhesives. J. Adhes. 13: $3 ; 1981$.

Khoury, F. A. Polymer science and engineering: challenges, needs, and opportunities. National Research Council Report; 1981.

Khoury, F. A. Workshop proceedings: morphology of polyethylene \& cross-linked polyethylene. 1981 Apri1; Gaithersburg, MD. EPRI Publ. \#EL-2134-LD; 1981 November.

Khoury, F. A. Physics vade mecum. Am. Phys. Soc.; Chapter 13; 1981.

Lang, S. B.; Davis, G. T.; DeReggi, A. S.; Broadhurst, M. G. Effects of poling field \& time on pyroelectric coefficient \& polarization uniformity in polyvinyl fluoride. Ferroelectrics 33: $119 ; 1981$.

Misra, D. N.; Bowen, R. L. Interaction of zinc ions with hydroxylapatite: adsorption from aqueous solutions. New York, NY: Plenum Press; 1981.

Peterlin, A. Tensile failure of crystalline polymers. J. Macromol. Sci. Phys. 19: 401; 1981.

Schroeder, L. W. ; Jordan, T. H. ; Brown, W. E. Raman spectrum from a single crystal of $\mathrm{SnHPO}_{4}$ Spectrochim. Acta Part A 37: 21; 1981.

Senich, G. A. Inverse gas chromatography, a method for studying polymer-migrant interactions in polyolefin packaging materials. CHEMTECH 11: 360; 1981.

Tesk, J. A. Solders and fluxes, chapter in Dentist's desk reference: materials, instruments, and equipment, lst Ed. Chicago, IL: American Dental Association. 1981.

Waterstrat, R. M. Binary phase diagrams of transition elements. Bull. Alloy Phase Diagrams. 2: 1981. [Excerpt published quarterly by American Society for Metals as part of the ASM/NBS Data Program for Alloy Phase Diagrams.]

Waterstrat, R. M. Analysis of selected alloys in the systems $\mathrm{Cr}-\mathrm{Pd}, \mathrm{Cr}-\mathrm{Ru}, \mathrm{V}-\mathrm{Pd}$, and Ta-Pt. J. Less-Common Met. 80: 31; 1981.

Weeks, J. J.; Clark, E. S.; Eby, R. K. Crystal structure of the low temperature phase (II) of polytetrafluoroethylene. Polymer 22: 1480; 1981. 
Weeks, J. J.; Eby, R. K.; Clark, E. S. Disorder in the crystal structures of phases I and II of copolymers of tetrafluoroethylene and hexafluoropropylene. Polymer 22: 1496; 1981.

Barnes, J. D. A computer controlled apparatus for gas transmission measurement. Technical Papers Vol. XXVIII, Society of Plastics Engineers, Inc. 1982. p. 19.

Bowen, R. L. Composite and sealant--past, present, and future. Pediatr. Dent. 4 1: 10; 1982.

Bowen, R. L. ; Rapson, J. E. ; Dickson, G. Hardening shrinkage and hygroscopic expansion of composite resins. J. Dent. Res. 61(5): 654; 1982.

Broadhurst, M. G. Electric-field induced changes in the phase and orientation of polyvinylidene fluoride crystals. Proceedings of the 28th IUPAC macromolecular symposium; 1982 July; Amherst, MA; International Union of Pure and Applied Chemistry. 1982; p. 455.

Broadhurst, M. G.; Davis, G. T.; DeReggi, A. S.; Roth, S. C.; Collins, R. E. Pyroelectricity and charge transport in a copolymer of vinylidene fluoride and tetrafluoroethylene. Polymer 23: 22; 1982.

Brown, D. W.; Lowry, R. E.; Smith, L. E. Hydrolytic degradation of polyester polyurethanes containing carbodiimides. Macromolecules 15: 453; 1982.

Brown, D. W.; Lowry, R. E.; Smith, L. E. Equilibrium acid concentration in hydrolyzed polyester diols \& polyester polyurethanes. Polym. Prepr. $23 ; 1982$.

Brown, D. W. ; Lowry, R. E. ; Smith, L. E. Prediction of the long term stability of polyester-based recording media. Nat. Bur. Stand. (U.S.) NBSIR 82-2530; 1982.

Bur, A. J. Fabrication and characterization of ultra-drawn thick PVDF transducers. Proceedings of the 28th IUPAC macromolecular symposium; 1982 July; Amherst, MA; International Union of Pure and Applied Chemistry. 1982 ; p. 457.

Chang, S. S. Migration of low molecular weight components from polyolefins. Proceedings of the 28th IUPAC macromolecular symposium; 1982 July; Amherst, MA; International Union of Pure and Applied Chemistry. 1982; p. 734.

Chang, S. S.; Bernstein, G.; West, E. D. ; Ditmars, D. A.; Ishihara, S. Enthalpy and heat capacity standard reference material--synthetic sapphire $\left(\alpha-\mathrm{Al}_{2} \mathrm{O}_{3}\right)$ from 10 to $2250 \mathrm{~K}$. J. Res. Nat. Bur. Stand. (U.S.) 87: 159; 1982

Chang, S. S.; Senich, G. A.; Smith, L. E. Migration of low molecular weight additives in polyolefins and copolymers. Nat. Bur. Stand. (U.S.) NBSIR 82-2472; 1982 March.

Crissman, J. M.; McKenna, G. B.; Khoury, F. A. Creep and stress-relaxation behavior of ultra high molecular weight polyethylene in uniaxial extension and compression. Society of Plastics Engineers Technical Papers, Vol. XXVIII; San Francisco, CA: SPE/ANTEC, 1982 May 9-13.

Davis, G. T.; Furukawa, T.; Lovinger, A. J.; Broadhurst, M. G. Structural and dielectric investigation on the nature of the transition in a copolymer of vinylidene fluoride \& trifluoroethylene (52/48 mol \%). Macromolecules 15: 329; 1982.

Dehl, R. E.; Grant, W. G. ; Cassel, J. M. Characterization of porosity in porous polymeric implant materials. Nat. Bur. Stand. (U.S.) NBSIR 81-2459; 1982 February.

Dickens, B. Thermally degrading polyethylene studied by means of factor-jump thermogravimetry. J. Polym. Sci. 20: 1065; 1982.

Dickens, B. Thermal degradation study of isotatic polypropylene using factor-jump thermogravimetry. J. Polym. Sci. 20: 1169; 1982.

Dickens, B. Vapor composition profiles estimated for thermally degrading polyethylene. Thermochim. Acta 55: 217; 1982.

Eby, R. K. Disorders in the crystal structure of homo- and co-polymers of polytetrafluoroethylene. Proceedings of the 28th IUPAC macromolecular symposium; 1982 July; Amherst, MA; International Union of Pure and Applied Chemistry. 1982; p. 592.

Eby, R. K. ; Smith, L. E.; Peterlin, A.; Broadhurst, M. G. ; Fanconi, B. M.; Cassel, J. M. ; Sanchez, I. C. Polymer science and standards division 1981. Nat. Bur. Stand. (U.S.) NBSIR 82-2452; 1982 March. 
Fanconi, B. M. Chain scission and mechanical degradation of polyethylene. Proceedings of the 28th IUPAC macromolecular symposium; 1982 July; Amherst, MA; International Union of Pure and Applied Chemistry. 1982; p. 34.

Fanconi, B. M.; Colson, J. P.; DeVries, K. L.; Smith, R. H. Free radicals and new end groups resulting from chain scission: II. mechanical degradation of polyethylene. Polymer 23: 1027; 1982.

Flynn, J. H. Lifetime prediction from polymer degradation kinetics. Proceedings of the 28th IUPAC macromolecular symposium; 1982 July; Amherst, MA; International Union and Pure and Applied Chemistry. 1982 ; p. 322.

Flynn, J. H. A collection of kinetic data for the diffusion of organic compounds in polyolefins. Polymer 23: $1325 ; 1982$.

Hosler, W. R. Electrical conductivity measurements on mixed ionic/electronic conducting materials at high temperatures. Proceedings of the 8 th symposium on thermophysical properties, 1981 June; Gaithersburg, MD. Nat. Bur. Stand. (U.S.) Spec. Pub1.; 1982.

Hunston, D. L.; Kinloch, A. J.; Shaw, S. J. Crack propagation in rubber toughened epoxies. Proceedings of the international conference on deformation, yield, and fracture; 1981; London, England: Plastics and Rubber Institute. 1982 ; p. 78.

Hunston, D. L.; Rushford, J. L. ; Wang, S. S.; Kinloch, A. J. Developing failure criteria for the polymers used in structural adhesives. Proceedings of the SPI conference on reinforced plastics/ composites; 1982 January 11-15; New York, NY: The Society of the Plastics Industry, Inc.; paper $29 \mathrm{C}$. 1982.

Khoury, F. A. The morphology of polymers. Polym. Prepr. Japan 31: 5; 1982.

Lovinger, A. J.; Davis, G. T.; Furukawa, T.; Broadhurst, M. G. Crystalline forms in a copolymer of vinylidene fluoride \& trifluoroethylene (52/48 mol \%). Macromolecules 15: $323 ; 1982$.

Mathew, M.; Kingsbury, P.; Takagi, S.; Brown, W. E. A new struvite-type compound, magnesium sodium phosphate heptahydrate. Acta Crystallogr. Sect. B 38: 40; 1982.

McCrackin, F. L.; Guttman, C. M.; Han, C. C. Monte Carlo calculations of the inverse radii of polymers in theta solvents and good solvents. Proceedings of the 28th IUPAC macromolecular symposium; 1982 July; Amherst, MA; International Union of Pure and Applied Chemistry. 1982; p. 524.

McKenna, G. B.; Penn, R. W. The mechanical durability of candidate elastomers for blood pump applications. Winter, G.; Plenk, H. ; Gibbons, D., eds. Proceedings of the 1st world biomaterials congress, 1980 April; Vienna, Austria. New York, NY: J. Wiley \& Sons, Ltd.; 1982.

McKinney, J. E. Apparatus for measuring wear of dental restorative materials. Wear 76: 337; 1982.

Mopsik, F. I.; DeReggi, A. S. Numerical evaluation of the dielectric polarization distribution from thermal pulse data. J. Appl. Phys. 53: 4333; 1982.

Paffenbarger, G. C.; Rupp, N. W. ; Patel, P. R. Copper-free amalgams: dimensional change after approximately five years at 60,37 and $23{ }^{\circ} \mathrm{C}$. J. Dent. Res. 61(6): 811; 1982.

Paffenbarger, G. C. ; Rupp, N. W.; Waterstrat, R. M. Metals in solution in mercury expressed from copper-rich dental amalgams. J. Dent. Res. 61(1): 30; 1982.

Passaglia, E. Relaxation of stresses in crazes at crack tips \& rate of craze extension. Polymer 23: $754 ; 1982$.

Peterlin, A. Dynamic viscosity of polymer solutions. Colloid. Polym. Sci. 260: 278; 1982.

Peterlin, A.; DeCandia, F.; Russo, R.; Vittoria, V. Mechanical and transport properties of drawn crosslinked low-denisty polyethylene. J. Polym. Sci. 20: 269; 1982.

Peterlin, A.; DeCandia, F.; Russo, R.; Vittoria, V. Shrinkage and retractive forces during the annealing of drawn low-density polyethylene. J. Polym. Sci. 20: 1175; 1982.

Rubin, R. J.; Weiss, G. H. Random walks on lattices: the problem of visits to a set of points revisited. J. Math. Phys. 23: 250; 1982.

Sanchez, I. C. Theory of polymer-polymer blend phase behavior. Kem. Ind. 31: 199; 1982. 
Sanchez, I. C. Thermodynamic properties of polymer blends. Proceedings of the 28th IUPAC macromolecular symposium; 1982 July; Amherst, MA; International Union of Pure and Applied Chemistry. 1982; p. 660.

Sanchez, I. C. Equation of state theories of polymer blends. Sole, Karl, ed. Proceedings of the international symposium on polymer compatibility: principals and practices, vol. 3. 1980 December; Midland, MI. Midland, MI: MMI Press; 1982 August; p. 201.

Senich, G. A. Chromatographic studies of diffusion in polymers. Proceedings of the 28th IUPAC macromolecular symposium; 1982 July; Amherst, MA; International Union of Pure and Applied Chemistry. 1982; p. 740 .

Takagi, S.; Mathew, M.; Brown, W. E. Water-rich hydrates: the structures of dimagnesium potassium hydrogenbis(arsenate) 15-hydrate and dimagnesium potassium hydrogenbis (phosphate) 15-Hydrate. Acta Crystallogr. Sect. B 38: 44; 1982.

VanderHart, D. L. ${ }^{13} \mathrm{C}$ NMR in oriented polymers. Proceedings of the 28th IUPAC macromolecular symposium; 1982 July; Amherst, MA; International Union of Pure and Applied Chemistry. 1982; p. 4.

VanderHart, D. L. Deductions about the morphology of wet and wet beaten celluloses from solid state ${ }^{13} \mathrm{C}$ NMR. Nat. Bur. Stand. (U.S.) NBSIR 82-2534; 1982.

VanderHart, D. L.; Earl, W. L. Measurement of ${ }^{13} \mathrm{C}$ chemical shifts in solids. J. Magn. Reson. 48: 35; 1982.

Young, R. A.; Brown, W. E. Structures of biological minerals, chapter in Biological mineralization and demineralization. Nancollas, G. H., ed. New York, NY: Springer-Verlag; 1982. p. 101.

Zapas, L. J. Nonlinear mechanical behavior of polymer solutions at various concentrations.

Proceedings of the 28th IUPAC macromolecular symposium; 1982 July; Amherst, MA; International Union of Pure and Applied Chemistry. 1982; p. 860.

Barnes, J. D. Standard reference materials for gas transmission measurements. ACS Symposium Series; in press.

Bowen, R. L.; Cobb, E. N.; Rapson, J. E. Adhesive bonding of various materials to hard tooth tissues. XXV: improvement in bond strength to dentin. J. Dent. Res.; in press.

Broadhurst, M. G.; Bur, A. J.; Schwartz, R. B. The effect of neutron irradiation on electric conductivity in cellulose acetate. Radiat. Prot. Dosim.; in press.

Crissman, J. M.; Zapas, L. J. The mechanical behavior of isotatic polypropylene subjected to various strain histories in uniaxial extension. Polymer; in press.

DeReggi, A. S.; Lang, S.; Mopsik, F. I. Preexisting polarization and influence of electrode materials on $\mathrm{PVF}_{2}$ electrics as determined by thermal pulse and pyroelectric methods. J. Appl. Phys.; in press.

Dermann, K.; Rupp, N. W. ; Brauer, G. M. Effect of hygrophilic diluents on the properties of cured composites. J. Dent. Res.; in press.

DeRossi, D.; DeReggi, A. S.; Broadhurst, M. G.; Roth, S. C.; Davis, G. T. Method of evaluating the thermal stability of the pyroelectric properties of polyvinylidene fluoride--effects of poling temperature \& field. J. Appl. Phys.; in press.

Dickens, B. Factor-jump thermogravimetry as applied to the study of polymer degradations, chapter in Advances in fundamentals of polymer stability \& degradation. Jellinick, H. H. G., ed. New York, NY: Elsevier Press; in press.

Dickens, B.; Florin, R. E. User's guide for the chemiluminescence apparatus. Nat. Bur. Stand. (U.S.) NBSIR; in press.

Dickens, B.; Flynn, J. H. Thermogravimetry applied to polymer degradation kinetics, chapter in Instrumental and physical characterization of macromolecules. Craver, C. D., ed. American Chemical Society Advances in Chemistry Series; in press.

Eby, R. K.; Davis, G. T.; Wang, F. W.; Broadhurst, M. G.; Fanconi, B. M.; Sanchez, I. C.; Cassel, J. M. Polymer science and standards division. Kobunshi Ronbunshu; in press.

Eby, R. K.; Davis, G. T.; Wang, F. W.; Broadhurst, M. G. ; Fanconi, B. M.; Sanchez, I. C.; Cassel, J. M. Polymer science and standards division. Polym. News; in press. 
Flynn, J. H. F Florin, R. E. Polymer degradation and pyrolysis mechanisms, chapter 4 in Polymer characterization using gas chromotography. New York, NY: Marcel Dekker; in press.

Han, C. C.: Amis, E. J. Cooperative and self-diffusion of polymers by dynamic light scattering in semidilute solutions. Polymer; in press.

Han, C. C.; Fufimoto, T.; Nagasawa, M.; Noda, I.; Choshl, H.; Furuhashi, H.; Matsushita, Y: Preparation and characterization of block copolymers of $\mathrm{H}$-styrene and D-styrene. Polym. J.; in press.

Han, C. C.; Schaefer, D. Application of photon correlation spectroscopy. Pecora, R., ed. New York, NY: Plenum Press; in press.

Hunston, D. L.; Rushford, J. L.; Newitt, W. R.; Vandreui1, B. A. Rheology of cure for intaglio printing inks. Org. Coat. Plast. Prepr.; in press.

Hunston, D. L.; Wang, S. S.; Kinloch, A. J. Characterization of fracture behavior of adhesive joints. Org. Coat. Plast. Prepr.; in press.

Kranbueh1, D. E.; Verdier, P. H. Interpretation of quasi-elastic light scattering data for flexible chains: model dependence. Polymer; in press.

Mabie, C. P.; Menis, D. L.; Whitenton, E. P.; Trout, R. L.; Metherate, R. S. ; Ferry, C. H. Gel-route preparation of low fusing dental porcelain frit. J. Biomed. Mater. Res.; in press.

Mabie, C. P.; Menis, D. L.; Whitenton, E. P.; Trout, R. L.; Metherate, R. S.; Ferry, C. H. Comparison of gel-route and commercially prepared low fusing dental porcelain frit. J. Biomed. Mater. Res.; in press.

McCrackin, F. L. ; Guttman, C. M. ; Han, C. C. Monte Carlo calculation of the hydro-dynamic radius at the $\theta$ point: deviations from analytical Gaussian behavior. Macromolecules; in press.

McKenna, G. B.; Zapas, L. J. Experiments on the small strain behavior of crosslinked natural rubber, parts I and II. Polymer; in press.

McKinney, J. E.; Wu, W. Relationship between subsurface damage and wear of dental restorative composites. J. Dent. Res.; in press.

McKinney, J. E.; Wu, W. Influence of chemfcal softening on wear of three types of dental components. J. Dent. Res.; in press.

Mopsik, F. I.; DeReggi, A. S. The poling behavior of polyvinylidene fluoride at room temperature. J. Appl. Phys.; in press.

Paffenbarger, G. C.; Rupp, N. W.; Patel, P. R. Dimensional changes on a high-tin content amalgam. J. Dent. Res.; in press.

Peterlin, A. Diffusion and permeability of polymers, chapter in Controlled drug delivery. CRC Press; in press.

Peterlin, A. Accordion type laser-Raman (ALR) scattering by polymers. J. Polym. Sci.; in press.

Peterlin, A. Polymer morphology \& mechanics: the strength \& stiffness of polymers. New York, NY: Marcel Dekker, Inc.; in press.

Phillips, J. C. Mechanical relaxation of liner materials in acetic acid. Nat. Bur. Stand. (U.S.) NBSIR; in press.

Phillips, J. C. Transport of acetic acid in polyethylene, Nat. Bur. Stand. (U.S.) NBSIR; in press. Phillips, J. C. ; Peterlin, A. Diffusion of ethyl acetate vapor in strained low density polyethylene. Polym. Eng. Sci.; in press.

Rubin, R. J.; Weiss, G. H. Random walks: theory and selected applications. Adv. Chem. Phys.; in press.

Sanchez, I. C. ; Poser, C. I. Surface thermodynamics of polymers: theory. Mittal, K. L., ed. Proceedings of the international symposium on physico-chemical aspects of polymer surfaces; 1981 August; New York, NY: American Chemical Society; in press.

Senich, G. A. A review of the migration of food-contact organotin stabilizers from poly(vinylchloride). Polymer; in press. 
Takagi, S.; Mathew, M.; Brown, W. E. The crystal structure of sodium strontium phosphate nonahydrate. Acta Crystallogr.; in press.

Tung, M. S.; Brown, W. E. Characterization \& modification of electrochemical properties of teeth. J. Dent. Res.; in press.

Vogel, G. L.; Chow, L. C.; Brown, W. E. A microanalytical procedure for the determination of calcium, phosphorus, and fluoride in enamel. Caries Res.; in press.

Wagner, H. L..; Dillon, J. G. A low-shear high temperature viscometer: the viscosity of ultrahigh molecular weight polyethylene. ACS Symposium Issue; in press.

Wang, F. W. ; Howe11, B. F. Fluorescence measurement of antioxidant migration from low-density polyethylene into 1-propanol. Proceedings of the ACS organic coatings and applied polymer science symposium; 1982 September; Kansas City, MO: American Chemical Society; in press.

Wang, F. W. ; Lowry, R. E. Measurement of polymer-polymer compatibility by non-radiative energy transfer technique. Polym. Prepr.; in press.

Wang, F. W. ; McCrackin, F. L. Analysis of ultracentrifugation interference patterns with image digitizer: application to molecular weight determination of SRM 1478 polystyrene. Polymer; in press.

Wu, W. Application of small angle $x$-ray technique in damaged particulate reinforced composites: a theoretical treatment. Polymer; in press.

Wu, W. Small angle neutron scattering of partially segregated polymer blends. Polymer; in press.

Wu, W.; McKinney, J. E. Influence of chemicals on wear of dental composites. J. Dent. Res.; in press.

\section{Sponsored Conferences}

Symposium on Random Walks and Their Applications to the Physical and Biological Sciences June 28-July 1, 1982 at NBS, Gaithersburg, MD

\section{External Recognition}

Poster Recognition

Sybron/Kerr "Milestones in Dentistry--1891-1981

January 1982

R. L. Bowen

Honorary Membership

Omicron Kapper Upsilon Honor Dental Society

May 1982

R. L. Bowen

1982 Clemson Award for Contributions to the Technical Literature

April 1982

R. L. Bowen

Honorary Membership

Zeta Zeta Chapter for Omicron Kappa Upsilon

Georgetown University

May 1981

J. M. Casse 1

Irwin Vigness Memorial Award for 1982 from the Institute for Environmental Sciences for a paper on a fiber optics strain gauge

S. Edelman

North American Thermal Analysis Society

October 1981

J. H. Flynn, Fellow

Royal Netherlands Academy of Sciences

Corresponding Membership

August 1982

H. P. R. Frederikse 
Plastics $\mathrm{Hall}$ of Fame

June 1982

Frank W. Re inhart

\section{Patents}

Tertiary Aromatic Amine Accelerators Derived from Para-amino Phenethanol in Vinyl Monomers H. Argentar

August 18, 1981

U. S. Patent $4,284,551$

Method for Preparing Microporous Glassy Filler Grains for Dental Resin Composites C. P. Mabie and D. L. Menis

December 22, 1981

U. S. Patent $4,306,913$

Improved Dental Material

C. P. Mabie and D. L. Menis

Filed January 22, 1982

Combinations of Sparingly Soluble Calcium Phosphates in.Slurries and Pastes as Mineralizers and Cements W. E. Brown and L. C. Chow

Filed April 26, 1982 


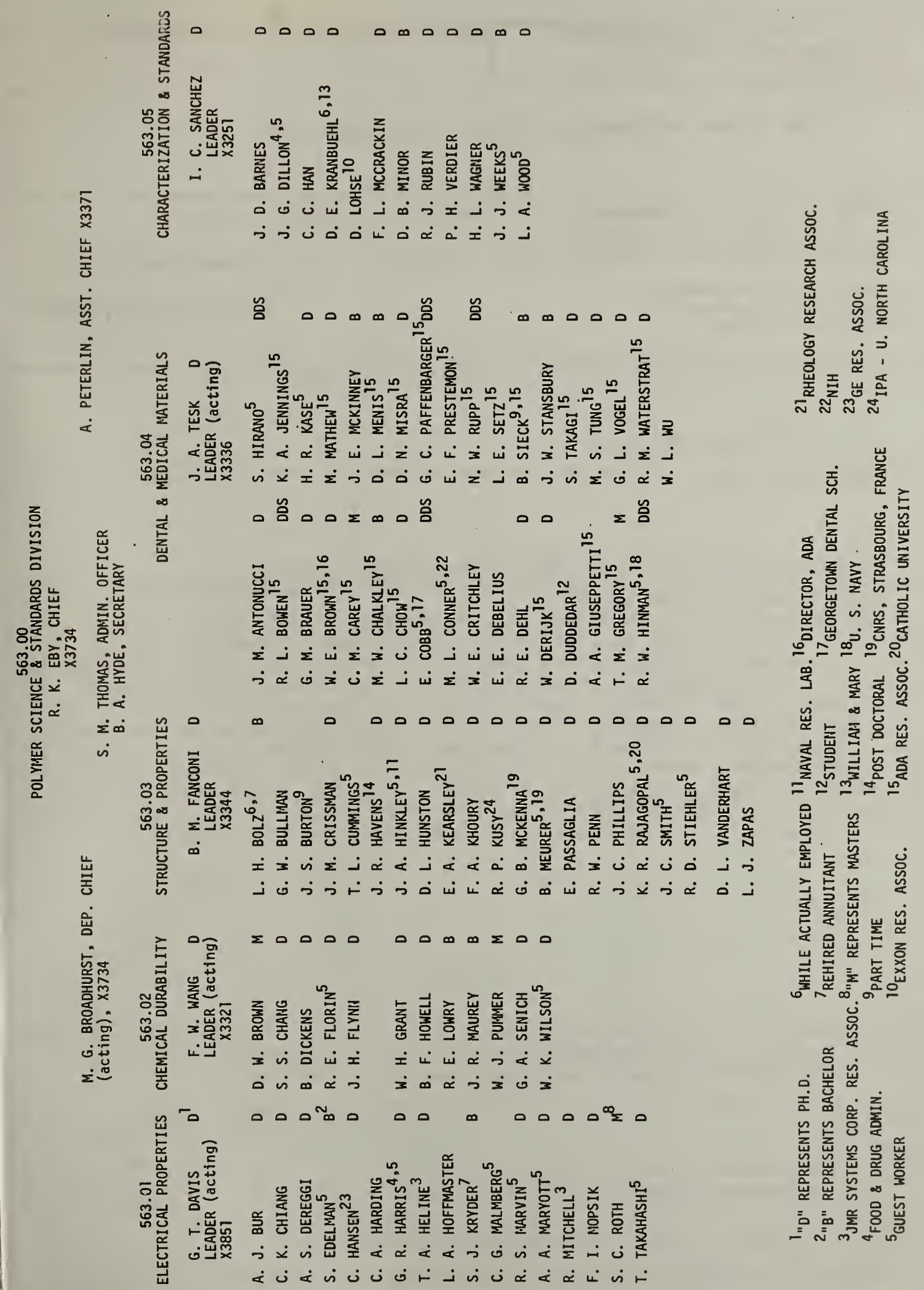


NBS.114A (REV. 20001

\begin{tabular}{|c|c|}
\hline UIBLIOGRAPHIC DATA & 1. PUBLICATION OR \\
REPORT NO. \\
SHEET (See instructions) & NBSIR $82-2607$ \\
\hline
\end{tabular}

2. Performlns Orgen. Report Noy 3. Publlcation Date
March 1983

4. TITLE AND SUBTITLE

Polymer Science and Standards Division Annual Report 1982

5. AUTHORS:) K. Eby, M. G. Broadhurst, G. T. Davis, F. W. Wang, B. M. Fanconi, J. M. Cassel, I. C. Sanchez

\begin{tabular}{l|l|}
\hline 6. PERFORMING ORGANIZATION (If jolnt or other than NBS, see Instructions) & 7. Contracu Grant No. \\
NATIONAL BUREAU OF STANDAROS & \\
DEPARTMENT OF COMMERCE & 8. TYPo of Report \& Perlod Covered \\
WASHINGTON, D.C. 20234 &
\end{tabular}

9. SPONSORING ORGANIZATION NAME AND COMPLETE ADDRESS (Street, Clty, Stote, ZIP)

NBS

Washington, DC 20234

10. SUPPLEMENTARY NOTES

Document describes a computer program; SF-185, FIPS Software Summary, is attached.

11. ABSTRACT (A 200-word or less factual summary of most significant information. If document inciudes a significant bibilography or literature survey, mention it here)

Although synthetic polymers have been used as materials of technology for only 3 quarters of a century, they have left little of our economy, technology, industry, science \& culture untouched. We have moved rapidly into an age in which an overgrowing number of humanity's needs are served by polymers. The volume currently produced exceeds that of steel \& forms the basis of industries which add over $\$ 90$ billion of value by manufacturer (a measure of the relative economic importance of manufacturing among industries) and provides 3.4 million jobs. Recent summaries show that polymers and polymer composite research already accounts for about $47 \%$ of the total industrial R\&D expenditure for metals, polymers, \& inorganic materials. Among these materials, polymers also constitute about $39 \%$ of the value added by manufacturer, $49 \%$ of the jobs, $45 \%$ of the number of scientific publications, and $39 \%$ of the ASTM standards.

12. KEY WORDS (Six to tweive entries; aiphabetical order; capitalize oniy proper names; and separate key words by semicolons) Chemical durability; characterization; dental and medical materials; dielectric plastics; performance; polymers; mechanical durability; migration.

13. AVAILABILITY

XX) Unlimised

For Official Distribution. Do Not Release to NTIS

$\square$ Order From Superintendent of Documents, U.S. Government Printing Office, Washington, D.C. 20402.

14. NO. OF

PRINTED PAGES 98 100

15. Price

$\$ 11.50$

Order From National Technical Information Service (NTIS), Springfield, VA. 2216I 
